\title{
Copyright
}

by

Marco Antonio Iglesias-Hernandez

2008 
The Dissertation Committee for Marco Antonio Iglesias-Hernandez certifies that this is the approved version of the following dissertation:

\section{An Iterative Representer-Based Scheme for Data Inversion in Reservoir Modeling}

Committee:

Clint Dawson, Supervisor

Todd Arbogast

Steven Bryant

Omar Ghattas

Hector Klie 


\title{
An Iterative Representer-Based Scheme for Data Inversion in Reservoir Modeling
}

\author{
by \\ Marco Antonio Iglesias-Hernandez, B.S.; M.S.
}

\section{Dissertation}

Presented to the Faculty of the Graduate School of

The University of Texas at Austin

in Partial Fulfillment

of the Requirements

for the Degree of

Doctor of Philosophy

The University of Texas at Austin

August 2008 
To my parents

Julieta Hernandez Cabrera

and

Marco A. Iglesias Flores. 


\section{Acknowledgments}

I would like to express my deepest gratitude to Professor Clint Dawson for his exceptional guidance during my graduate studies. His active interest in my education and career have provided invaluable support and motivation for me.

I wish to thank Professors Todd Arbogast, Steven Bryant, Omar Ghattas and Dr. Hector Klie for his helpful comments and suggestions on my dissertation.

I would like to acknowledge Dr. John Baird for being my student mentor during my first two years in the CAM program. I also thank Dr. Mauricio Santillana for his friendship which have made the last year much more enjoyable.

Marco Antonio Iglesias-Hernandez

The University of Texas at Austin

August 2008 


\section{An Iterative Representer-Based Scheme for Data Inversion in Reservoir Modeling}

Marco Antonio Iglesias-Hernandez, Ph.D.

The University of Texas at Austin, 2008

Supervisor: Clint Dawson

With the recent development of smart-well technology, the reservoir community now faces the challenge of developing robust and efficient techniques for reservoir characterization by means of data inversion. Unfortunately, classical history-matching methodologies do not possess computational efficiency and robustness needed to assimilate data measured almost in real time. Therefore, the reservoir community has started to explore techniques previously applied in other disciplines. Such is the case of the representer method, a variational data assimilation technique that was first applied in physical oceanography.

The representer method is an efficient technique for solving linear inverse problems when a finite number of measurements are available. To the best of our knowledge, a general representer-based methodology for nonlinear inverse problems has not been fully developed. We fill this gap by presenting a novel implementation of the representer method applied to the nonlinear inverse problem of identifying petrophysical properties in reservoir models. Given production data from wells and prior knowledge of the petrophysical properties, the goal of our formulation is to find improved parameters so that the reservoir model prediction 
fits the data within some error given a priori.

We first define an abstract framework for parameter identification in nonlinear reservoir models. Then, we propose an iterative representer-based scheme (IRBS) to find a solution of the inverse problem. Sufficient conditions for convergence of the proposed algorithm are established. We apply the IRBS to the estimation of absolute permeability in singlephase Darcy flow through porous media. Additionally, we study an extension of the IRBS with Karhunen-Loeve (IRBS-KL) expansions to address the identification of petrophysical properties subject to linear geological constraints. The IRBS-KL approach is compared with a standard variational technique for history matching.

Furthermore, we apply the IRBS-KL to the identification of porosity, absolute and relative permeabilities given production data from an oil-water reservoir. The general derivation of the IRBS-KL is provided for a reservoir whose dynamics are modeled by slightly compressible immiscible displacement of two-phase flow through porous media. Finally, we present an ad-hoc sequential implementation of the IRBS-KL and compare its performance with the ensemble Kalman filter. 


\section{Contents}

Acknowledgments $\quad$ v

$\begin{array}{lll}\text { Abstract } & \text { vi }\end{array}$

List of Tables $\quad$ xi

List of Figures $\quad$ xii

Chapter 1 Introduction 1

1.1 Motivation . . . . . . . . . . . . . . . . . 1

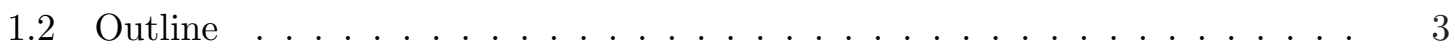

Chapter 2 Literature Review 5

2.1 History Matching . . . . . . . . . . . . . . . . 5

2.2 Data Assimilation . . . . . . . . . . . . . . . . 6

2.3 Data Assimilation for History Matching . . . . . . . . . . . . . . 8

2.4 History matching under geological constraints . . . . . . . . . . . . . . 9

Chapter 3 Data Inversion in Reservoir Models $\quad 10$

3.1 Mathematical Motivation for Data Inversion . . . . . . . . . . . . . . . 10

3.2 Prior Information . . . . . . . . . . . . . . . . . . . 11

3.2.1 Preliminaries and Notation . . . . . . . . . . . . . . 11

3.2 .2 The Forward Model . . . . . . . . . . . . . . . . . . . . 12

3.2.3 Measurements and Parameters Prior Knowledge . . . . . . . . . . . 13

3.3 The solution to the Inverse Problem . . . . . . . . . . . . . . . . . . . 14

3.3.1 An Iterative Representer-Based Scheme (IRBS) . . . . . . . . . . . . 15

3.3.2 Step (3). Solution to the linearized problem by the representer method 15

3.3 .3 Convergence Properties . . . . . . . . . . . . . . . . . 19

Chapter 4 A Toy Model 22

4.1 An application of the abstract framework . . . . . . . . . . . . 23

4.2 Implementation . . . . . . . . . . . . . . . . . . 27

4.2.1 Discretization . . . . . . . . . . . . . . . . . . . 29

4.2 .2 Measurement Functional . . . . . . . . . . . . . . . . . . . 30 
4.2.3 Prior Error Information and Norms _. . . . . . . . . . . . . . 30

4.2.4 Generation of Synthetic Data . . . . . . . . . . . . . . . . . . 30

4.2 .5 Stopping rule . . . . . . . . . . . . . . . 31

4.2.6 The Groundwater Flow . . . . . . . . . . . . . . . . . . 31

4.2.7 Single-phase Darcy flow through porous media . . . . . . . . . . 33

$\begin{array}{lll}\text { Chapter } 5 & \text { The IRBS with geological constraints } & 40\end{array}$

5.1 Parametrization by K-L expansions . . . . . . . . . . . . . . . . . . . 40

5.2 The IRBS with K-L parametrization . . . . . . . . . . . . . . . . . . . . 43

5.2.1 Some Remarks on the IRBS-KL . . . . . . . . . . . . . . 44

$5.2 .2 \quad$ A Numerical Example . . . . . . . . . . . . . . . . . . . 45

5.3 The IRBS vs. a standard Gradient-Based Technique . . . . . . . . . . . 50

5.3.1 The standard Variational Approach . . . . . . . . . . . . . . 50

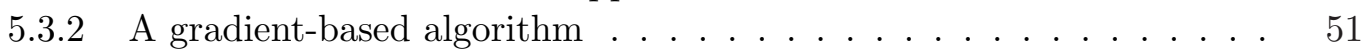

5.3 .3 Remarks on Algorithm 3. . . . . . . . . . . . . . . . . . 52

5.3 .4 Numerical Comparison . . . . . . . . . . . . . . . . 52

$\begin{array}{lll}\text { Chapter } 6 & \text { IRBS in Two-Phase flow } & \mathbf{5 4}\end{array}$

6.1 The Forward Model . . . . . . . . . . . . . . . . . . 54

6.2 Data and Measurement Functionals . . . . . . . . . . . . . . 58

6.3 The IRBS for Two-Phase flow . . . . . . . . . . . . . . . . 60

6.3.1 The Euler-Lagrange Equations . . . . . . . . . . . . . . . 61

6.3 .2 Definitions and Notation . . . . . . . . . . . . . . 65

6.4 Implementation . . . . . . . . . . . . . . . . . . 66

6.5 Numerical Experiments. Waterflood . . . . . . . . . . . . . 68

6.5.1 Estimation of Absolute and Relative Permeabilities . . . . . . . . 68

6.5.2 Estimation of Porosity . . . . . . . . . . . . . . . . . 70

6.6 A Sequential Implementation . . . . . . . . . . . . . . . . . . . . . . 73

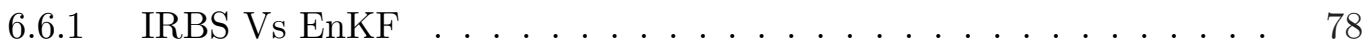

$\begin{array}{lll}\text { Chapter } 7 & \text { Conclusions and Future Research } & 90\end{array}$

$\begin{array}{ll}\text { Appendix A Proofs of Chapter } 3 & 93\end{array}$

A.1 Proof of Lemma $2 \ldots \ldots \ldots \ldots \ldots$

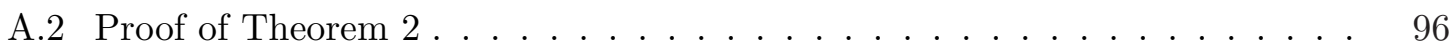

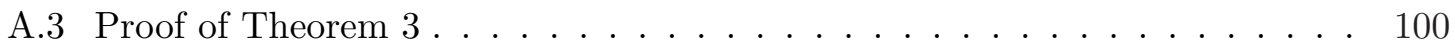

$\begin{array}{lr}\text { Appendix B Two-Phase flow Derivations } & 104\end{array}$

B.1 Reduction of the Linearized Case . . . . . . . . . . . . . . . . . . . 104

B.1.1 Measurement Functional on the linearized variables . . . . . . . . 107

B.1.2 The EL equations . . . . . . . . . . . . . . . . . . . 108

B.2 The Representers Algorithm . . . . . . . . . . . . . . . . . . . 112

$\begin{array}{lr}\text { Bibliography } & 116\end{array}$ 


\section{List of Tables}

4.1 Information for data assimilation. Experiment I. . . . . . . . . . . . . . . 33

4.2 Numerical Results. Experiment I. . . . . . . . . . . . . . . . . . . . . 33

4.3 Reservoir description. Experiment II and Experiment III. . . . . . . . . . . 35

4.4 Prior error information for Experiment II. . . . . . . . . . . . . . . 35

4.5 Numerical Results. Experiment II. . . . . . . . . . . . . . . . . . . . . 37

4.6 Data Assimilation Information. Experiment IIIA and IIIB. . . . . . . . . . 38

5.1 Reservoir description. Experiments IV $(\mathrm{A})-\mathrm{IV}(\mathrm{C}) \ldots \ldots \ldots$

5.2 Numerical Results. Experiment IV. . . . . . . . . . . . . . . . . 47

6.1 Reservoir description. Experiment V and VI. . . . . . . . . . . . . . 69

6.2 Convergence Results. Experiment V. . . . . . . . . . . . . . . . . . . 72

6.3 Information for data assimilation. Experiment V. . . . . . . . . . . . . 73

6.4 Convergence Results. Experiment VI. . . . . . . . . . . . . . . . . 73

6.5 Information for data assimilation. Experiment V . . . . . . . . . . . 78

6.6 Convergence Results. Experiment VII(a). . . . . . . . . . . . . . . . 84 


\section{List of Figures}

4.1 Experiment I. Configuration and $\log$ hydraulic conductivities. . . . . . . . . 34

4.2 Experiment II. $\log$ permeability fields. . . . . . . . . . . . . . . 36

4.3 Experiment III. $\log$ permeability fields. . . . . . . . . . . . . . . . . . 38

4.4 Experiment III. Error performance. . . . . . . . . . . . . . . . . . . . 39

5.1 Experiment IV. Stochastic realizations. . . . . . . . . . . . . . . 47

5.2 Example of an stochastic realization with KL parametrization . . . . . . . 48

5.3 Experiment IV. Permeability Fields. . . . . . . . . . . . . . . . . . 49

5.4 Experiment IV (c). Performance of cost functional . . . . . . . . . . . 53

6.1 Experiment V. Configuration and $\log$ permeability fields . . . . . . . . . 70

6.2 Experiment V. History Matching. . . . . . . . . . . . . . . . . . 71

6.3 Experiment V. Relative permeability curves. . . . . . . . . . . . . . 72

6.4 Experiment V(b). Porosity fields. . . . . . . . . . . . . . . . . 74

6.5 Experiment VI(a)-(b). Water cut (WCT) history matching. Wells P1-P2. . 75

6.6 Experiment VI(a)-(b). Water cut (WCT) history matching. Wells P3-P4. . 76

6.7 Experiment VI. SPE log-permeability and experimental semivariograms. . . 78

6.8 Experiment VI. Initial log-permeability and variograms. . . . . . . . . . . . 79

6.9 Experiment VI. Stochastic realizations for Experiment VII. . . . . . . . . . 80

6.10 Experiment VI. Log permeability fields. . . . . . . . . . . . . . . . . . . 81

6.11 Experiment VII(a). BHP history matching. . . . . . . . . . . . . 82

6.12 Experiment VII(a). Total flow rate history matching. . . . . . . . . . . 83

6.13 Experiment VI(b).EnKF estimated log permeability fields. . . . . . . . . . 85

6.14 Experiment VI(b). EnKF History-Matching . . . . . . . . . . . . . 86

6.15 EnKF History-Matching (total flow rate). . . . . . . . . . . . . . 88

6.16 EnKF History-Matching (bottom hole pressure). . . . . . . . . . . . 89 


\section{Chapter 1}

\section{Introduction}

\subsection{Motivation}

The main challenge of reservoir management is to achieve the maximum hydrocarbon recovery under the minimum operational costs. This goal cannot be accomplished without a reservoir simulator capable of predicting the reservoir performance. However, the reliability of reservoir models depends on the accuracy of the knowledge of the subsurface properties. These properties are highly heterogeneous and can be measured only at few locations. Therefore, in order to obtain an accurate reservoir model, it is essential to develop techniques that allow us to obtain a better characterization of the petrophysical properties of the subsurface.

Automatic history matching is one of the aspects involved in the characterization of the petrophysical properties of a reservoir. The aim of history matching is to obtain estimates of the petrophysical properties, so that the model prediction fits the observed production data. Traditionally, data acquisition for history matching was available by well-logging techniques which were expensive and required well intervention. However, after 1997 [27], the development of smart-well technology has dramatically changed the restrictive scenario for data acquisition. With the aid of down-hole permanent sensors, it is possible to monitor production data in almost real-time. This data may be used for history matching in order to obtain a better characterization of the reservoir which in turn can be used to optimize recovery. Unfortunately, classical history matching techniques are not able to process large sets of data under reasonable computational cost. For this reason, the reservoir community has started to implement techniques that have been previously used in other areas. Such is the case in physical oceanography and meteorology [11, 25, 69, 18, 58], where several data assimilation technologies have been developed to overcome analogous 
problems to those faced by the reservoir community.

Within the broad methods for data assimilation, we distinguish between two main approaches that have been applied to the reservoir modeling application: the Monte Carlotype or probabilistic framework [23] and the variational approach [11]. The former consists of generating an ensemble of different possible realizations of the state variable with given prior distribution. The model is run for each member of the ensemble up to the first measurement time. Then, each state variable is updated with standard formulas of Kalman filtering theory [40] to produce a posterior distribution which is now used to continue the process. In the Kalman filter-type methods, the model simulator is used as a black box without the need of an adjoint code. This feature offers a significative advantage in history matching for which several applications of the Ensemble Kalman Filter (EnKF) have been conducted $[22,30,19,31,63,48,47,50,51]$. However, there are still open questions arising from the EnKF implementation. Unphysical solutions, overestimation and poor uncertainty quantification have been reported with the EnKF [31, 30]. Despite attempts to remediate these undesired outcomes, the nonlinearity of reservoir models clearly goes beyond the capabilities of the EnKF.

In the variational approach, the solution is sought as a minimizer of a weighted leastsquares functional that penalizes the misfit between model-based and real measurements. The corresponding minimization involves the implementation of an optimization technique which typically requires the development of an adjoint code for the efficient computation of the derivatives of the cost functional. It is worth mentioning that many variational approaches for history-matching have been studied during the last years. However, those approaches are suboptimal and computationally expensive because of the highly-nonlinear and large-scale reservoir models. For this reason, variational "data assimilation" techniques have received attention for reservoir and groundwater applications $[5,6,4,36,67,55]$. In linear variational "data assimilation" an inexpensive minimization of a weighted least-squares cost functional can be conducted by the so-called representer method [11]. Assuming that the measurement space is finite dimensional, the representer method provides the solution to the Euler-Lagrange equations (E-L) resulting from the variational formulation. For nonlinear problems, the representer method cannot be implemented directly. Nevertheless, some representer-based techniques have been explored to compute an approximation to the solution of the E-L equations $[4,55]$. However, these approaches do not necessarily yield a direction of descent of the cost functional. Therefore, an additional technique such as line search is needed to accelerate or even obtain convergence $[4,67,55]$. Unfortunately, these additional implementations may compromise the efficiency required for reservoir applications. 
In this work, we present a novel implementation of the representer method for the identification of petrophysical properties in reservoir models by means of data inversion. Given production data and prior knowledge of the "uncertain" property of the reservoir, a solution to the identification problem is the limit of a sequence of solutions to affine inverse problems solved by the representer method. At every iteration step, information from the previous iteration is utilized to pose a linearized inverse problem defined by the minimization of a weighted least-squares functional that penalizes the misfit between model prediction and production data. Additionally, this functional contains a regularization term that incorporates prior knowledge of the reservoir property of interest. Furthermore, the aforementioned minimization is constrained by the solution to the linearization of the reservoir (forward) model.

By applying standard techniques from iterative regularization theory [32, 21], convergence results are obtained for the proposed iterative representer-based scheme (IRBS). Furthermore, we extend the proposed methodology to study the identification of reservoir properties under lineal geological constraints. Both analytical and numerical results show that this methodology is efficient and robust in the estimation of petrophysical properties in reservoir models. Moreover, comparisons with a standard gradient-based approach and the state-of-the art Ensemble Kalman Filter method, show that the IRBS is a promising tool for history-matching in reservoir models.

\subsection{Outline}

A general overview of classical history matching techniques will be provided in Chapter 2 . We also discuss some data assimilation methods, originally implemented in oceanography and meteorology, that have recently been applied for reservoir characterization. In Chapter 3 , we develop an abstract formulation for the problem of identifying poorly known petrophysical properties in reservoir models. We propose an iterative representer-based scheme (IRBS) to find a solution to the identification problem. Additionally, we conduct the general considerations for the application and convergence of the IRBS. In Chapter 4 we study the application of the IRBS to the single-phase Darcy flow through porous media. We establish conditions for which the IRBS provides a solution to the identification of absolute permeability. In Chapter 5 we study the IRBS under geological constraints with Karhunen-Loeve expansions. A comparison with a standard gradient based approach is conducted. In Chapter 6 we extend this algorithm for the simultaneous estimation of porosity and absolute permeability given pressure and saturation data from the immiscible displacement of twophase compressible fluids. The derivation of the representer method is provided. A reduced 
estimation problem is posed where capillarity and gravity are neglected. We present some numerical experiment for the estimation of absolute and relative permeability as well as porosity. Finally, in Chapter 7 the results of the thesis are summarized and some future research directions are proposed. 


\section{Chapter 2}

\section{Literature Review of Parameter Estimation in Reservoir Modeling}

In the last four decades extensive scientific and technical advances have been achieved in history matching techniques. It is not possible to cover all these methodologies so a brief overview will be provided in the following paragraphs.

\subsection{History Matching}

Before 1972, automatic history matching was approached with a least-squares formulation. The revolutionary study of Jacquard and Jain [37] consists of dividing the reservoir into zones of constant permeability and minimizing the sum of squared pressure residuals. Despite this remarkable work, the minimization of a least-squares criterion was not enough to ensure stability and uniqueness of the solution. In addition, the computational demand for computing sensitivity coefficients imposed severe restrictions for the number of zones resulting in poor reconstruction of the heterogeneity of a real permeability field.

A considerable improvement in history matching took placed with the introduction of optimal control for the minimization of the least-squares criteria. Chavent et al. [16] used an adjoint method to reduce the computational cost for the inverse estimation of absolute permeability and the skin factor in a single-phase flow model. However, the need for a regularization of the least-squares problem was first realized by Korganoff [41]. He added regularization terms to penalize oscillatory solutions that arise when increasing the number of permeability zones.

In 1976, the important work of Gavalas et al. [29] addressed the problem with a Bayesian approach where the parameters are treated as random variables with known mean 
and prior error covariance. The statistical smoothing effect alleviates numerical instabilities of the parameter estimates. It should be mentioned that the need for well known prior error statistics was the main drawback for this technique since efficient measurement technologies were not available at that time.

An alternative history matching method for the case where prior information is not available was studied by Kravaris and Seinfield [42]. Following the regularization theory for ill-posed problems that Tikhonov introduced in 1963 [66], they developed a theory on distributed parameter identification. Their results focused on the estimation of permeability in single-phase flow through porous media. Tai-young Lee and Seinfield [43] extended those results for the simultaneous estimation of absolute and relative permeabilities given rates and pressure from the immiscible displacement of two-phase flow. Further development of the theory of parameter identification was made with the implementation of iterative regularization techniques. For instance, Hanke [32] has derived an iterative LevenbergMarquardt method for permeability identification in a single-phase fluid.

In recent years geostatistics has played an important role for reservoir characterization. For instance, given some measurements of the permeability obtained by routine core analysis, it is possible to generate a stochastic simulation of the permeability field. Therefore, the Bayesian approach has been preferable for history matching purposes since a trustworthy prior guess can be obtained. However, the need for more efficient and less expensive optimization techniques has been the subject of recent research. For example, Li et al. [44] have implemented the adjoint method for sensitivity of production data to permeability and skin factor in three-phase flow. Reynolds and Oliver [57] used the same technique and model for the simultaneous estimation of absolute and relative permeabilities.

\subsection{Data Assimilation}

At the same time history matching techniques were developed, the oceanography and meteorology communities were attempting to solve a similar problem. Ocean models are based on several assumptions and approximations that do not capture the system. Discrepancies between model circulations and observations exceed the standard error in observations. It was essential to derive "data assimilation" methods to obtain better estimates of the system given the model and observational data.

As in history matching, the data assimilation problem can be posed in a Bayesian framework. When the prior error covariances are Gaussian and the model dynamics linear the problem is equivalent to the minimization of a weighted-least squares objective functional. The minimizer is called the general smoother or generalized inverse and it can be 
estimated with classical optimization methods. As an alternative method for computing the generalized inverse, the representer method was introduced first by Bennett in 1985 [9]. One of the advantages of the representer method is that the Euler-Lagrange equations resulting from the first order optimality condition are solved exactly at the cost of $2 M+1$ model integrations where $M$ is the number of measurements. Data assimilation applications with the representer method have been used to study tropical ocean circulations [13]. It has also been applied to improve ocean tides given altimetry measurements from the TOPEX/POSEIDON radar satellite [20]. More recently, Rosmond and Xu [58] have used the representer method to increase the capabilities of the US Navy's data assimilation system.

Another implementation that was adopted by the assimilation community is the Kalman filter [40]. In this method the assimilation is performed sequentially and the state of the system is updated as soon as measurements become available. Instead of minimizing an objective functional, it computes the best linear unbiased estimate at each measurement time. It can be shown [25] that at the final time, the filter and the generalized inverse are the same. This feature makes the filtering ideal in forecasting applications. However, a drawback of this method is that an evolution equation for the covariance of the state is needed which is twice the dimension of the state variable.

For nonlinear dynamics, even if the model prior probability density function (pdf) is Gaussian, the pdf for the model evolution will become non-Gaussian and the Kalman filter formulas are neither valid nor useful for approximating the model outputs. The extended Kalman filter (EKF) is a linearized version of the Kalman filter that was established to address nonlinear data assimilation. After Evensen [25] showed some applications where the linearization for the error covariance led to numerical instabilities, in 1994 he [23] proposed the ensemble Kalman Filter (EnKF); a Monte Carlo version of the KF for data assimilation in nonlinear dynamics. In this method, an ensemble of state vectors is generated and the model is evolved until measurements become available. Then, the analysis step is performed where each ensemble is updated with the standard Kalman filter formula but with Gaussian errors added to the observations [14]. It has been shown that when the problem is nearly Gaussian, the Kalman filter captures the correct posterior covariance, which is approximated by computing the ensemble. Therefore, one of the advantages of the EnKF is that there is no evolution equation to solve for the error covariance of the state.

The EnKF has been validated on several models [25] and is currently used in real applications. For example, it is the data assimilation technique used by the system TOPAZ (Towards an Operational Prediction system for the North Atlantic European coastal Zones) which is in operation since 2004 . 


\subsection{Data Assimilation for History Matching}

The ability to obtain reliable data from the field was a key for success of data assimilation in oceanography and meteorology. In the recent years, new technologies became available for the reservoir community with the introduction of so-called smart wells equipped with sensors for monitoring downhole pressure and valves to control rates. The reservoir community has become interested in migrating data assimilation techniques for reservoir characterization.

In the EnKF, the forward model is used as a black-box and only a relatively simple code is needed for data assimilation. For this reason, the EnKF has received more attention for reservoir applications. In 2003 Naevdal [50] introduced for the first time the EnKF for the estimation of absolute permeability given production data. Then, in $[48,31,71]$ several applications of the EnKF to the PUNQ-S3 model were conducted. It is worth mentioning that in $[50,31]$, an overshooting of the updated petrophysical properties was observed after several times of assimilation. Another issue regarding the implementation of the EnKF is the estimation of unphysical solutions. Such is the case of water saturation which typically follows a non-gaussian distribution. To overcome this difficulty [30] proposed to use the EnKF update over a transformation of the physical variable. A different approach, proposed in [68] and used by [30], consists on updating the dynamic and static variables with the standard EnKF formulation. Then, if a dynamic estimates is non-physical, the corresponding static ensemble member is used to generate, by rerunning the forward model, a consistent dynamic replicate.

In addition to the implementation of the EnKF for the assimilation of production data, in $[19,63]$ seismic data has also been utilized. Since $4 \mathrm{D}$ seismic data can be given as a difference between two surveys, $[19,63]$ combined the EnKF and the ensemble Kalman smoother for the assimilation of production and seismic data respectively. Furthermore, the EnKF has been used for history matching of location of geologic facies [47]. In addition, the EnKF has been considered as an integral part of the so called closed-loop optimization [49].

In contrast, the representer method has received limited attention for reservoir applications. In ground water modeling the represented method have been applied for state and parameter estimation [56, 67]. Furthermore, Baird and Dawson [5, 6] have conducted an a priori and a posterior error analysis of the representer method for state estimation in single-phase flow. They have also implemented the representer method for state estimation in two-phase flow [4]. For permeability and state estimation Iglesias and Dawson [36] have studied an implementation of the representer method in single-phase Darcy flow. In addition, the recent work of Przybysz-Jarnut [55] has used the representer method for the 
permeability estimation from pressure data in two-phase flow. Finally, the methodology proposed in this dissertation has been introduced in [35].

\subsection{History matching under geological constraints}

The history matching community has recognized the importance of developing history matching techniques that allow the incorporation of geological constraints. Several approaches have been followed to accomplish this goal. For example, Sarma et. al [61] used truncated Karhunen-Loeve expansions to parameterize geological constraints for model updating in a reservoir management closed-loop implementation. A parametrization of multipoint geostatistics based on kernel principal component analysis (PCA) was utilized in [59]. Analogously, the discrete cosine transform (DCT) has been recently applied in [39] to parameterize absolute permeability. This parametrization was coupled to the EnKF for history matching in an oil-water reservoir [38] .

A different approach to history matching under geological constraints can be followed with geostatics. For example, the gradual deformation method [15] produces stochastic perturbations of the initial (Gaussian) prior geological model until the corresponding updated model generates flow responses that match production data. For non-Gaussian fields, a local perturbation method was proposed by Srinivasan and Bryant in [64]. In this paper, the authors addressed computational efficiency by proposing a parallel domain decomposition algorithm to conduct the local perturbation. Recently, Barrera [7] developed a geologically consistent history matching technique. Barrera applied an stochastic perturbation method

to calibrate flow functions consistent with a geological model simulated from different rock types (pore networks). 


\section{Chapter 3}

\section{An Iterative Approach to Data Inversion in Reservoir Models.}

This chapter establishes the general mathematical framework of an iterative representerbased scheme for data inversion in reservoir modeling.

\subsection{Mathematical Motivation for Data Inversion}

In order to motivate the definition of the IRBS, let us consider a reservoir whose porosity and permeability are denoted by $\phi, K$ respectively. Assume that the reservoir is filled with a single-phase fluid of viscosity $\mu$ and (constant) compressibility $c$. In addition, suppose that there are some injection/production sources denoted by $f$. Under the aforementioned assumptions, the dynamics of the reservoir can be predicted by the slightly-compressible single-phase Darcy flow through porous medium model [8] which is mathematically described by the following equation

$$
c \phi \frac{\partial p}{\partial t}-\frac{1}{\mu} \nabla \cdot K \nabla p=f
$$

where $p$ is the pressure which defines the state of the reservoir. When $c, \mu, \phi, K$ are given and some initial and boundary conditions are prescribed, then (3.1) can be solved uniquely for $p$ in the appropriate mathematical spaces [46]. In this model $c$ and $\mu$ are (constant) properties of the fluid which may be easily obtained in practical applications. In contrast, $\phi$ and $K$ are petrophysical properties of the reservoir which may dramatically change with respect to position within reservoir. Then, a small number of measurements from wells, may not be enough to capture the heterogeneities of those properties. In other words, $\phi$ and $K$ 
are "uncertain" in the sense that they are a poorly known. Therefore, if those parameters were utilized in (3.1), the solution would be different from the pressure measurements collected at the wells. Therefore, we need to address the following inverse problem. Given measurements of the state, find improved parameters such that the discrepancy between model prediction and real measurements is "small". Any methodology to solve the inverse problem requires precise information about the following prior information:

(1) Structure of the (forward) model;

(2) Measurement process;

(3) Observational error information;

(4) Parameters prior knowledge.

In the following sections we mathematically define the inverse problem within a general abstract framework of Hilbert spaces. In addition, assumptions on the prior information (1)-(4) are developed. Then, an iterative representer-based scheme is proposed to find a solution to this problem. Finally, we establish conditions under which the IRBS will converge to a solution to the inverse problem.

\subsection{Prior Information}

\subsubsection{Preliminaries and Notation}

For any vector $v \in \mathbb{R}^{N}$ we denote by $\|v\|=\left(\sum_{i=1}^{N}\left|v_{i}\right|^{2}\right)^{1 / 2}$ the Euclidian norm. For $A \in \mathbb{R}^{N \times N}$ we also denote by $\|A\|=\sup _{\|v\| \neq 0} \frac{\|A v\|}{\|v\|}$. For any symmetric positive definite matrix $C \in \mathbb{R}^{N \times N}$, we denote by $\|v\|_{C}=\left(v^{T} C v\right)^{1 / 2}$ the corresponding induced norm in $\mathbb{R}^{N}$. Given a Hilbert space $\mathcal{H}$, we denote the closed ball centered at $k$ of radius $r$ by $B(r, k)$. Furthermore, the inner product of $\mathcal{H}$ is denote by $\langle\cdot, \cdot\rangle_{\mathcal{H}}$. Given $\Omega \subset \mathbb{R}^{N}$ and $0<T<\infty$, we define $\Gamma=\partial \Omega, \Omega_{T}=\Omega \times(0, T)$ and $\Gamma_{T}=\Gamma \times(0, T)$. We consider [46] the usual Sobolev spaces $H^{2}(\Omega)$ with the norm given by $\|u\|_{H^{2}(\Omega)}=\left(\sum_{|\alpha| \leq 2} \int_{\Omega}\left\|D^{\alpha} u\right\|^{2}\right)^{1 / 2}$, and $H^{1}\left(0, T ; L^{2}(\Omega)\right)$ with the norm $\|u\|_{H^{1}\left(0, T ; L^{2}(\Omega)\right)}=\left(\int_{0}^{T}\|u(t)\|_{L^{2}(\Omega)}^{2} d t\right)^{1 / 2}$. Additionally, we define the space $H^{2,1}\left(\Omega_{T}\right)=L^{2}\left(0, T ; H^{2}(\Omega)\right) \cap H^{1}\left(0, T ; L^{2}(\Omega)\right)$ with the norm $\|u\|_{H^{2,1}\left(\Omega_{T}\right)}=\left(\int_{0}^{T}\|u(t)\|_{H^{2}(\Omega)}^{2} d t+\|u\|_{H^{1}\left(0, T ; L^{2}(\Omega)\right)}^{2}\right)^{1 / 2}$. Finally, we define $H^{1 / 2,1 / 4}\left(\Gamma_{T}\right)=$ $L^{2}\left(0, T ; H^{1 / 2}(\Gamma)\right) \cap H^{1 / 4}\left(0, T ; L^{2}(\Omega)\right)$ with the norm $\|u\|_{H^{1 / 2,1 / 4}\left(\Gamma_{T}\right)}=\left(\int_{0}^{T}\|u(t)\|_{H^{1 / 2}(\Gamma)}^{2} d t+\right.$ $\left.\|u\|_{H^{1 / 4}\left(0, T ; L^{2}(\Gamma)\right)}^{2}\right)^{1 / 2}$ where $H^{1 / 2}(\Gamma)$ and $H^{1 / 4}\left(0, T ; L^{2}(\Gamma)\right)$ are interpolation spaces defined in the sense of Definition 2.1 in [46]. 


\subsubsection{The Forward Model}

Let us represent the reservoir by an open bounded set $\Omega \subset \mathbb{R}^{l}$ with $l=2,3$. Given some $0<T<\infty$, suppose that $[0, T]$ is the time of interest for the analysis of the reservoir dynamics. Assume that the reservoir has $N$ uncertain parameters. The corresponding parameter space is defined by $\mathcal{K}=\Pi_{i=1}^{N} \mathcal{K}_{i}$ where each $\mathcal{K}_{i}$ is a linear subspace either of $L^{2}(\Omega)$ or $L^{2}\left(\Omega_{T}\right)$. Let $\mathcal{S}$ and $\mathcal{Z}$ be arbitrary subspaces of $L^{2}\left(\Omega_{T}\right)$. The set $S$ is the space of all possible states of the reservoir. We now assume that the dynamics of the reservoir can be described, over a finite interval of time $[0, T]$, by a set of (forward) model equations

$$
G(k, s)=0 .
$$

where $G: \mathcal{K} \times \mathcal{S} \rightarrow \mathcal{Z}$ is a nonlinear operator. For a fixed parameter $k \in \mathcal{K}$, an element of the state space $s(x, t) \in \mathcal{S}$ that satisfies (3.2) is the corresponding state of the reservoir which simulates the reservoir dynamics over the interval of time. Parameters which are not considered uncertain in the sense of the preceding paragraph are assumed to be contained in the functional relation chosen for the operator $G$. The subspaces $\mathcal{S}, \mathcal{K}$ and $\mathcal{Z}$, in addition to the functional relation for $G$ must be defined for the particular reservoir model of interest. For the following analysis consider the following assumptions on the (forward model) operator $G$

(F1) $G: \mathcal{K} \times \mathcal{S} \rightarrow \mathcal{Z}$ is continuously Frechet-differentiable;

(F2) For each $k \in \mathcal{K}$, there exists a unique $s \in \mathcal{S}$ with $G(k, s)=0$;

(F3) For all $(\tilde{k}, \tilde{s}) \in \mathcal{K} \times \mathcal{S}$, that satisfies $G(\tilde{k}, \tilde{s})=0$, we assume $D_{s} G(\tilde{k}, \tilde{s}): \mathcal{S} \rightarrow \mathcal{Z}$ is a linear isomorphism, and in addition, for every $r>0$ and $k_{*} \in \mathcal{K}$ we assume that there exists a constant $C_{1}$ such that for every $k \in B\left(r, k_{*}\right)$ and $s \in \mathcal{S}$ that satisfy $G(k, s)=0$, the solution $\delta s$ to

$$
D_{k} G(k, s) \delta k+D_{s} G(k, s) \delta s=0
$$

satisfies

$$
\|\delta s\|_{\mathcal{S}} \leq C_{1}\|\delta k\|_{\mathcal{K}}
$$

for every $\delta k \in \mathcal{K}$ (note that $C_{1}$ is the same for every $k \in B(r, \tilde{k})$ ). 


\subsubsection{Measurements and Parameters Prior Knowledge}

Each measurement is treated independently at each location and time of collection. Let us suppose that we are given a vector $d^{\eta}=\left[d_{1}^{\eta}, \ldots, d_{M}^{\eta}\right]$ of $M$ measurements of the state of the reservoir, such that each $d_{m}^{\eta}$ was collected at $\left(x_{m}, t_{m}\right)$. In general, measurements are corrupted by noise. In other words, the vector of measurements $d^{\eta}$ is only an approximation to the measurements of the state, that is

$$
\left\|d^{\eta}-d\right\|_{C^{-1}} \leq \eta
$$

where $d=\left[d_{1}, \ldots, d_{M}\right]$ is the vector of perfect (noise-free) measurements. The observation process responsible for a perfect measurement $d_{m}$ is represented, on the space $\mathcal{S}$, by the action $\left(\mathcal{L}_{m}(s)=d_{m}\right)$ of linear functionals $\mathcal{L}_{m}: \mathcal{S} \rightarrow \mathbb{R}$. In addition, we assume that not only $d^{\eta}$ is provided but also its associated error covariance matrix $C$. Furthermore, prior knowledge information for each uncertain parameter $k_{i}$ is required to be given as a prior mean $\bar{k}_{i}$ and the corresponding prior error covariance function $C_{k_{i}}$. By definition of the covariance operator [65], $C_{k_{i}}: \mathcal{K}_{i} \rightarrow \mathcal{K}_{i}$ is positive definite. Let us define the formal inverse in the sense of Tarantola:

$$
\int_{\Sigma} C_{k_{i}}(\xi, \chi) C_{k_{i}}^{-1}\left(\chi, \xi^{\prime}\right) d \chi=\delta\left(\xi-\xi^{\prime}\right)
$$

where $\Sigma$ is either $\Omega$ or $\Omega_{T}$. It is not difficult to see that

$$
\langle\phi, \psi\rangle_{C_{k_{i}}} \equiv \int_{\Sigma} \int_{\Sigma} \phi(\xi) C_{k_{i}}^{-1}\left(\xi, \xi^{\prime}\right) \psi\left(\xi^{\prime}\right) d \xi d \xi^{\prime}
$$

defines an inner product on $\mathcal{K}_{i}$ for each $i=1, \ldots, N$. In summary, the following information must be provided:

(1) $d^{\eta}=\left[d_{1}^{\eta}, \ldots, d_{M}^{\eta}\right]$ a vector of $M$ (possibly corrupted by noise) measurements of the state of the reservoir at $\left\{\left(x_{m}, t_{m}\right)\right\}_{m=1}^{M}$;

(2) The matrix $C$ of measurement error covariance (positive definite);

(3) Prior knowledge of parameters $\bar{k}=\left[\bar{k}_{1}, \ldots, \bar{k}_{N}\right]$;

(4) Parameters prior error covariance operators $\left\{C_{k_{1}}, \cdots, C_{k_{N}}\right\}$;

(5) Expressions for the linear functionals $\mathcal{L}=\left[\mathcal{L}_{1}, \ldots, \mathcal{L}_{M}\right]$ representing the process from which $d=\left[d_{1}, \ldots, d_{M}\right]$ was obtained.

The following assumption is needed. 
Assumption 1 (A1). For $i=1, \ldots, N$ the space $\mathcal{K}_{i}$ is complete with the induced norm $\|\cdot\|_{C_{k_{i}}}$

Then, from standard functional analysis $\mathcal{K}=\Pi_{i=1}^{N} \mathcal{K}_{i}$ is a Hilbert space with the norm induced by the following inner product.

$$
\langle\phi, \psi\rangle_{\mathcal{K}} \equiv \sum_{i=1}^{N}\left\langle\phi_{i}, \psi_{i}\right\rangle_{C_{k_{i}}}
$$

In the context of reservoir modeling (Model 3.1 for example), the prior error covariance reflects the prior knowledge of the spatial variability of the petrophysical properties. In real applications, the covariance may be determined from well logs, core analysis, seismic and the geology of the formation.

\subsection{The solution to the Inverse Problem}

Having established the assumption on the prior information, we need now to define what we mean by a solution to the inverse problem. Inspired by the theory of parameter identification $[32,21]$, we now formulate

Definition 1. Assume that the state of the system is described by (3.2) and the measurement process is represented by $\mathcal{L}=\left[\mathcal{L}_{1}, \ldots, \mathcal{L}_{M}\right]$ as described above. Given a vector $d=\left[d_{1}, \ldots, d_{M}\right]$ of exact measurements (noise-free) of the state of the system, a solution to the Noise-Free Parameter Identification Problem (NF-PIP) is an element $k \in \mathcal{K}$, such that

$$
G(k, s)=0 \quad \text { and } \quad \mathcal{L}(s)=d
$$

for some $s \in \mathcal{S}$.

It is important to emphasize that we need to have measurements from the real state of the reservoir. Suppose that the measurements were perfect (noise-free) and the model dynamics and measurement process are represented correctly by the mathematical models under consideration. Then it is reasonable to think that the measurements were obtanied from the "true state" $s_{T}$ of the system which satisfies the model dynamics (3.2) for some "true parameter" $k_{T}$. For this reason we postulate the following:

Attainability condition of data: There exists at least one solution $k_{T} \in \mathcal{K}$ to the noisefree parameter identification problem. We refer to $k_{T}$ as the "true parameter". 
For example, suppose we are interested in the identification of $K$ in model (3.1) given pressure data from some well locations (assuming that $c, \phi$ and $\mu$ are perfectly known). It is clear that there may be several permeability configurations that will generate the same pressure response which is measured at the wells. However, the reservoir has one and only one permeability field which is responsible for the generation of the collected measurements.

\subsubsection{An Iterative Representer-Based Scheme (IRBS)}

In order to find an approximation to a solution of the NF-PIP (Definition (1)), we propose the following algorithm. Assume we are given $k^{0}=\bar{k}$, an estimate of the error level $\eta$, prior error covariances $C,\left\{C_{k_{i}}\right\}_{i=1}^{N}$ and some $\tau>1$.

Algorithm 1 (IRBS). For $n=1, \ldots$

(1) Solution to the forward model. Given $k^{n-1}$, we compute $s_{F}^{n}$ the solution to the forward model

$$
G\left(k^{n-1}, s_{F}^{n}\right)=0
$$

(2) Stopping rule (Discrepancy Principle). If $\left\|d^{\eta}-\mathcal{L}\left(s_{F}^{n}\right)\right\| \leq \tau \eta$, stop. Output: $k^{n-1}$.

(3) Parameter update by means of data assimilation with representers. Define $k^{n}$ as the solution to the linearized data assimilation problem

$$
J(s, k)=\left[d^{\eta}-\mathcal{L}(s)\right]^{T} C^{-1}\left[d^{\eta}-\mathcal{L}(s)\right]+\left\|k-k^{n-1}\right\|_{\mathcal{K}}^{2} \rightarrow \min
$$

subject to

$$
L G(k, s)=D_{k} G\left(k^{n-1}, s_{F}^{n}\right)\left[k-k^{n-1}\right]+D_{s} G\left(k^{n-1}, s_{F}^{n}\right)\left[s-s_{F}^{n}\right]=0 .
$$

In the following sections we develop sufficient conditions for convergence of this algorithm.

\subsubsection{Step (3). Solution to the linearized problem by the representer method}

Now we prove that problem (3.11)-(3.12) admits a unique solution. Moreover, an explicit formula for $k$ will be provided in terms of "representers". Note that (3.12) is nothing but the 
linearization of the reservoir model around $\left[k^{n-1}, s_{F}^{n}\right]$. The first term of $(3.11)$ is a weighted least-squares functional that penalizes the misfit between real and predicted measurement. The second term accounts for the error between prior and estimated parameters.

\section{The "Representers"}

We define the following variables: $\Delta k=k-k^{n-1}$ and $\Delta s=s-s_{F}^{n}$. Then, problem (3.11)-(3.12) is equivalent to the minimization of

$$
J^{n}(\Delta s, \Delta k)=\left[d^{\eta}-\mathcal{L}\left(s_{F}^{n}\right)-\mathcal{L}(\Delta s)\right]^{T} C^{-1}\left[d^{\eta}-\mathcal{L}\left(s_{F}^{n}\right)-\mathcal{L}(\Delta s)\right]+\|\Delta k\|_{\mathcal{K}}^{2}
$$

subject to the linearized constraint

$$
D_{k} G\left(k^{n-1}, s_{F}^{n}\right) \Delta k+D_{s} G\left(k^{n-1}, s_{F}^{n}\right) \Delta s=0 .
$$

We now state the following

Lemma 1. Assume (A1) and (F1)-(F3) hold. Let $r>0$ and $k_{*} \in \mathcal{K}$ arbitrary. Take $C_{1}$ given by (F3) for $B\left(r, k_{*}\right)$. If $k^{n-1} \in B\left(r, k_{*}\right)$, then, for each $m \in\{1, \ldots, M\}$, there exists a "representer" $\gamma_{m}^{n} \in \mathcal{K}$ such that

$$
\left\langle\gamma_{m}^{n}, \Delta k\right\rangle_{\mathcal{K}}=\mathcal{L}_{m}(\Delta s)
$$

for all $\Delta k \in \mathcal{K}$, where $\Delta s$ is the solution to (3.14). Furthermore, the "representer matrix" defined by

$$
\left[R^{n}\right]_{i j}=\left\langle\gamma_{i}^{n}, \gamma_{j}^{n}\right\rangle_{\mathcal{K}}
$$

satisfies

$$
\left\|R^{n}\right\| \leq C_{1}^{2}\|\mathcal{L}\|^{2}
$$

with $\|\mathcal{L}\|=\sup _{i}\left\|\mathcal{L}_{i}\right\|$.

Proof: By the invertibility of $D_{s} G\left(k^{n-1}, s_{F}^{n}\right)$ we may consider the linear operator $\mathcal{W}^{n}: \mathcal{K} \rightarrow \mathcal{S}$ such that $\mathcal{W}^{n}(\Delta k)=\Delta s$ where $\Delta s$ is the solution to (3.14). Since $k^{n-1} \in$ $B\left(r, k_{*}\right)$, by hypothesis (F3) there exists $C_{1}$ such that

$$
\left\|\mathcal{W}^{n}(\Delta k)\right\|_{\mathcal{S}}=\|\Delta s\|_{\mathcal{S}} \leq C_{1}\|\Delta k\|_{\mathcal{K}}
$$


for every $\Delta k \in \mathcal{K}$. Then, by linearity and continuity of $\mathcal{L}_{i} \circ \mathcal{W}^{n}$ we are allowed to apply the Riez representation theorem. Thus, for each $i=1, \ldots, M$, we assume the existence of $\gamma_{i}^{n}$ such that

$$
\left\langle\gamma_{i}^{n}, \Delta k\right\rangle_{\mathcal{K}}=\left[\mathcal{L}_{i} \circ \mathcal{W}^{n}\right](\Delta k)=\mathcal{L}_{i}(\Delta s)
$$

where $\Delta s$ and $\Delta k$ are related by (3.14). Then, we have proved (3.15). On the other hand, from definition (3.16), (3.18) and (3.19) it follows that

$$
\left|\left[R^{n}\right]_{i j}\right| \leq\left\|\gamma_{i}^{n}||_{\mathcal{K}}\right\| \gamma_{j}^{n}\left\|_{\mathcal{K}} \leq\right\| \mathcal{L}_{i}\left|\left\||| \mathcal{L}_{j}\right\|\left\|\mathcal{W}^{n}\right\|^{2} \leq C_{1}^{2}\|\mathcal{L}\|^{2}\right.
$$

which proves (3.17).

The following theorem establishes local existence and uniqueness of the $n$th step of the IRBS.

Theorem 1. Assume (A1) and (F1)-(F3) hold. Let $r>0$ and $k_{*} \in \mathcal{K}$ arbitrary. Take $C_{1}$ given by (F3) for $B\left(r, k_{*}\right)$. If $k^{n-1} \in B\left(r, k_{*}\right)$ and the set of "representers" $\left\{\gamma_{m}^{n}\right\}_{m=1}^{M}$ is linearly independent, then $R^{n}$ is positive definite and

$$
k(\xi)=k^{n-1}(\xi)+\left[\gamma^{n}(\xi)\right]^{T}\left[R^{n}+C\right]^{-1}\left[d^{\eta}-\mathcal{L}\left(s_{F}^{n}\right)\right]
$$

is the unique minimizer of $J^{n}$ (3.11) subject to (3.12) in $\mathcal{K}$, where $\gamma^{n}(\xi)=\left[\gamma_{1}^{n}(\xi), \ldots, \gamma_{M}^{n}(\xi)\right]$.

Proof: From Lemma 1 we assume the existence of the representers $\left\{\gamma_{m}^{n}\right\}_{m=1}^{M}$. Clearly, (3.16) implies that $R^{n}$ is positive semidefinite. Moreover, if $x R^{n} x=0$ for some $x=\left(x_{1}, \ldots, x_{M}\right) \in \mathbb{R}^{M}$, then, from (3.16) and the linearity of the inner product it follows that

$$
\sum_{m, j}^{M} x_{j} R_{j, m}^{n} x_{m}=\left\langle\sum_{j=1}^{M} x_{j} \gamma_{j}^{n}, \sum_{m=1}^{M} x_{m} \gamma_{m}^{n}\right\rangle_{\mathcal{K}}=\left\|\sum_{j=1}^{M} x_{j} \gamma_{j}^{n}\right\|_{\mathcal{K}}^{2}=0
$$

Since by hypothesis $\left\{\gamma_{m}^{n}\right\}_{m=1}^{M}$ is linearly independent, we conclude that $x=0$ and so $R^{n}$ is positive definite.

Now we consider the following decomposition of $\mathcal{K}: \mathcal{K}=\operatorname{span}\left\{\gamma^{n}\right\}+\operatorname{span}\left\{\gamma^{n}\right\}^{\perp}$ where, $\operatorname{span}\left\{\gamma^{n}\right\} \equiv\left\{k \in \mathcal{K} \mid k=\sum_{m=1}^{M} \beta_{m} \gamma_{m}^{n}\right.$ for some $\left.\left\{\beta_{i}\right\}_{i=1}^{M} \in \mathbb{R}^{M}\right\}$. Then, for every $\Delta k \in \mathcal{K}$,

$$
\Delta k=\sum_{m=1}^{M} \beta_{m} \gamma_{m}^{n}+b
$$


where

$$
\left\langle\gamma_{i}^{n}, b\right\rangle_{\mathcal{K}}=0
$$

for all $i=1, \ldots, M$. If we substitute (3.23) into (3.19) and apply (3.24) we find $\left\langle\gamma_{i}^{n}, \Delta k\right\rangle_{\mathcal{K}}=$ $\sum_{j} \beta_{j}\left\langle\gamma_{i}^{n}, \gamma_{j}^{n}\right\rangle_{\mathcal{K}}=\left[\mathcal{L}_{i} \circ \mathcal{W}^{n}\right](\Delta k)=\mathcal{L}_{i}(\Delta s)$. Therefore, from definition (3.16)

$$
R^{n} \beta=\mathcal{L}(\Delta s)
$$

where $\mathcal{L}=\left[\mathcal{L}_{1}, \ldots, \mathcal{L}_{M}\right]$ and $\beta=\left[\beta_{1} \ldots, \beta_{M}\right]$. We now substitute (3.25) and (3.23) into (3.13)-(3.14). Then we use (3.24) and the definition of the representer matrix (3.16) to find that the minimization problem is posed in terms of

$$
J(\beta, b)=\left[d^{\eta}-\mathcal{L}\left(s_{F}^{n}\right)-R^{n} \beta\right]^{T} C^{-1}\left[d^{\eta}-\mathcal{L}\left(s_{F}^{n}\right)-R^{n} \beta\right]+\beta^{T} R^{n} \beta+\|b\|_{\mathcal{K}}^{2}
$$

over the set $\mathcal{C}=\mathbb{R}^{M} \times\left\{b \in \mathcal{K} \mid\left\langle\gamma_{i}^{n}, b\right\rangle=0\right\}$. Since $R^{n}$ is positive definite, it is not difficult to see that $J^{n}$ is a strict convex functional. Additionally it is clear that $\mathcal{C}$ is a convex subset of $\mathbb{R}^{M} \times \mathcal{K}$. Then, it follows from standard optimization theory that any local minimum of $J^{n}$ is a unique global minimum over $\mathcal{C}$. Therefore, we proceed to compute the first order optimality condition which characterizes a global minimizer over $\mathcal{C}$. Note that

$$
\frac{1}{2} D J^{n}(\beta, b)[\delta \beta, \delta b]=-\left[d^{\eta}-\mathcal{L}\left(s_{F}^{n}\right)-R^{n} \beta\right]^{T} C^{-1} R^{n} \delta \beta+\beta^{T} R^{n} \delta \beta+\langle b, \delta b\rangle_{\mathcal{K}}
$$

From (3.16) it is obvious that $R^{n}$ is positive definite. Since by definition $C$ is also positive definite, then $R^{n}$ and $R^{n}+C$ are invertible. Therefore, $D J^{n}=0$ implies

$$
(\beta, b)=\left(\left[R^{n}+C\right]^{-1}\left[d^{\eta}-\mathcal{L}\left(s_{F}^{n}\right)\right], 0\right) .
$$

We now use (3.28) in (3.23) which in turn, by the change of variables defined at the beginning of this subsection yields (3.21).

Remark 1. It is essential to note that the results from Theorem 1 do not depend on the accuracy of the measurement vector $d^{\eta}$. Expression (3.21) can be used with $d^{\eta}$ replaced by $d$ (noise-free data). Analogously, the inverse of the covariance matrix may be replaced with any positive definite matrix. However, for practical applications, measurements will always be subject to errors and the error covariance may be available. For this reason we have weighted the measurement misfit (see (3.11)) with the inverse of the covariance matrix, as is typically done in standard Data Assimilation [11], where $J^{n}$ may be derived from a 
Bayesian formulation for the linearized problem.

Remark 2. The assumption of linear independence of the representers can be interpreted as a condition that prevents us from having redundant measurements.

\subsubsection{Convergence Properties}

The IRBS proposed in the previous section is equivalent to the Levenberg-Marquardt method derived by Hanke in [32]. However in our approach, we consider an implicit system (see equation (3.2)) instead of the parameter-to-observation map of [32]. The reason for this selection comes from the fact that the reservoir model is commonly described by a set of PDEs for which energy inequalities may be available. Second, we always assume that the observation space is finite dimensional so that the representer method can be applied. In contrast to [32], with the aforemention property we do not need to interpolate the data which always comes as a finite dimensional set. Third, in our formulation we incorporate prior knowledge which is relevant to the application. Despite some subtle differences between our methodology and the one presented in [32], in [35] we showed that our approach is a particular case of Hanke's work in the case of a fixed Levenberg-Marquardt parameter $\alpha=1$ and an appropriate selection of the parameter norm. However, we point out that in [32] the linearized inverse problem is solved with an standard implementation of the Levenberg-Marquardt whereas in the IRBS, we apply the representer method (see Theorem $1)$.

We now state some necessary conditions for convergence of Algorithm 1. First we need the following assumptions

Assumption 2 (A2). Let us denote by $G_{r}$ the residual of $G$ defined by the relation

$$
\begin{aligned}
G(k, s)=G(\tilde{k}, \tilde{s})+D_{k} G(\tilde{k}, \tilde{s})[k-\tilde{k}] & +D_{s} G(\tilde{k}, \tilde{s})[s-\tilde{s}] \\
& +G_{r}(k-\tilde{k}, s-\tilde{s}) .
\end{aligned}
$$

Given $\hat{k}$ fixed, there exists $C_{2}>0$ and $\delta>0$ such that if $k, \tilde{k} \in B(\delta, \hat{k})$ then

$$
\left\|\mathcal{L}\left(s_{r}\right)\right\| \leq C_{2}\|k-\tilde{k}\|_{\mathcal{K}}\|\mathcal{L}(s)-\mathcal{L}(\tilde{s})\|
$$

where $s_{r}$ is the solution to

$$
D_{s} G(\tilde{k}, \tilde{s}) s_{r}=G_{r}(k-\tilde{k}, s-\tilde{s})
$$

$s$ and $\tilde{s}$ are the (unique) solutions to $G(\tilde{k}, \tilde{s})=0=G(k, s)$. 
As we indicated earlier, in [35] we have shown that under some conditions, the IRBS is equivalent to the algorithm proposed in [32]. By showing that equivalence, sufficient conditions for convergence of the IRBS can be easily obtained. In this dissertation (see appendix) however, we conduct the complete proofs of convergence for the IRBS pointing out the technical differences with the work of Hanke [32].

The first result we need is

Lemma 2. Let $d^{\eta} \in \mathbb{R}^{M}$ ( $\eta$ fixed) and $C \in \mathbb{R}^{M \times M}$ be a positive definite matrix. Suppose assumptions (A1)-(A2) and (F1)-(F3) hold. For any $k_{*} \in \mathcal{K}$ and $r>0$, let $C_{1}$ be the constant in (F3) for the closed ball $B\left(r, k_{*}\right)$. Define

$$
\rho \equiv \frac{1}{C_{1}^{2}\|\mathcal{L}\|^{2}\|C\|^{-1}+1}
$$

Suppose that for some $n$, the following inequality is satisfied

$$
\left\|d^{\eta}-\mathcal{L}\left(s_{r}\right)\right\|_{C^{-1}} \leq \frac{\rho}{a}\left\|d^{\eta}-\mathcal{L}\left(s_{F}^{n}\right)\right\|_{C^{-1}}
$$

for some $a>1$, where $s_{r}$ is the solution to

$$
D_{k} G\left(k^{n-1}, s_{F}^{n}\right)\left[k_{*}-k^{n-1}\right]+D_{s} G\left(k^{n-1}, s_{F}^{n}\right)\left[s_{r}-s_{F}^{n}\right]=0
$$

If $k^{n-1} \in B\left(r, k_{*}\right)$, then $k^{n}$ defined by (3.21) for $d^{\eta}=d^{*}$, satisfies

$$
\left\|k_{*}-k^{n-1}\right\|_{\mathcal{K}}^{2}-\left\|k_{*}-k^{n}\right\|_{\mathcal{K}}^{2}>2 \rho^{2}\left[\frac{a-1}{a}\right]\left\|d^{*}-\mathcal{L}\left(s_{F}^{n}\right)\right\|_{C^{-1}}^{2}
$$

Proof. See appendix.

The previous lemma is needed to show that, when some conditions are satisfied, the sequence $k^{n}$ generated with the representer method (Theorem 1) at the $n$th iteration, is a better approximation than the corresponding estimate at the $(n-1)$ th iteration. Moreover, it can be shown that $k^{n}$ is Cauchy which by completeness of $\mathcal{K}$ implies that $k^{n}$ has a limit. Then by showing that this limit is a solution the NF-PIP, we can prove

Theorem 2. Let $d \in \mathbb{R}^{M}$ be the vector of exact measurements and $C \in \mathbb{R}^{M \times M}$ an arbitrary positive definite matrix with eigenvalues $\left\{\lambda_{i}\right\}_{i=1}^{M}$ and $\alpha \equiv \max \lambda_{i} / \min \lambda_{i}$. Let us assume (A1)-(A2) and (F1)-(F3) and let $k_{*}$ be a solution to the NF-PIP. For $\hat{k}=k_{*}$, take $C_{2}$ and $\delta>0$ as in (A2) . Consider $B\left(\delta / 2, k_{*}\right)$ and the corresponding constant $C_{1}$ as in (F3). Let $\rho$ be defined by (3.32). If $k^{0}=\bar{k}$ (the initial guess) satisfies

$$
\left\|k_{*}-\bar{k}\right\|_{\mathcal{K}}<\min \left\{\frac{\delta}{2}, a_{2}\right\}
$$


for some $a_{2}<\frac{\rho}{2 C_{2} \alpha^{1 / 2}}$, then $k^{n}$, defined in (3.21) for $d^{\eta}=d^{*}$, converges to a solution to the NF-PIP.

Proof. See appendix.

Theorem 2 is essential because it allows to prove convergence for exact measurements. In the case of noisy measurements, the first step is to show that the stopping rule terminates the iteration after a finite number of iterations. Then, we show that the sequence generated with Theorem 1, as the noise level converges to zero, the corresponding sequence converges to a solution to the NF-PIP granted by Theorem 2. In summary, we need to prove

Theorem 3 (Convergence of the IRBS). Let $d^{\eta}$ be a vector of noisy measurements for $\eta \in\left(0, \eta_{*}\right]$ where $\eta_{*}>0$ is the largest noise level given a priori. Let $C \in \mathbb{R}^{M \times M}$ be the (positive definite) covariance matrix corresponding to $d^{\eta_{*}}$. Let $\left\{\lambda_{i}\right\}_{i=1}^{M}$ be the eigenvalues of $C$ and we define $\alpha \equiv \max \lambda_{i} / \min \lambda_{i}$. Suppose that $\left\|d^{\eta}-d\right\|_{C^{-1}} \leq \eta$ where $d$ is the exact data. Let $k_{\eta}^{n}$ be defined by (3.21) and let $s_{F, \eta}^{n}$ be the solution to $G\left(k_{\eta}^{n}, s_{F, \eta}^{n}\right)=0$. Let us assume (A1)-(A2) and (F1)-(F3) and let $k_{*}$ be a solution to the NF-PIP. For $\hat{k}=k_{*}$, take $C_{2}$ and $\delta>0$ as in (A2). Consider $B\left(\delta / 2, k_{*}\right)$ and the corresponding constant $C_{1}$ as in (F3). Let $\tau>1 / \rho$ with $\rho$ defined by (3.32). If $k^{0}=\bar{k}$ satisfies

$$
\left\|k_{*}-k^{0}\right\|_{\mathcal{K}}<\min \left\{\delta / 2, \frac{\rho \tau-1}{\alpha^{1 / 2} C_{2}(1+\tau)}, \frac{\rho}{2 \alpha^{1 / 2} C_{2}}\right\}
$$

then (Discrepancy Principle) there exists a finite integer $n=n(\eta)$ such that $\left\|d^{\eta}-\mathcal{L}\left(s_{F}^{n(\eta)}\right)\right\|_{C^{-1}} \leq$ $\tau \eta$ and $\left\|d^{\eta}-\mathcal{L}\left(s_{F}^{m}\right)\right\|_{C^{-1}}>\tau \eta$ for $m<n(\eta)$. Moreover, $k_{\eta}^{n(\eta)}$ converges to a solution of the $N F-P I P$ as $\eta \rightarrow 0$.

Proof. See appendix.

With the convergence results presented in this chapter, we have established conditions on the forward model, measurement functional and prior information so that the IRBS will converge to an approximate solution in the sense of Definition 1. 


\section{Chapter 4}

\section{Application of the IRBS on a Linear Model}

In the previous section we presented a variational formulation for parameter identification in a system that is described by (3.2) where the operator $G$ satisfies hypothesis (F1)-(F3) and (A2). In this section we focus in a parabolic system described by the following operator $G: \mathcal{Y} \times H^{2,1}\left(\Omega_{T}\right) \rightarrow L^{2}\left(0, T ; L^{2}(\Omega)\right) \times H^{1 / 2,1 / 4}\left(\Gamma_{T}\right) \times H^{1}(\Omega):$

$$
G(Y, p)=\left[\begin{array}{c}
\frac{\partial p}{\partial t}-\nabla \cdot e^{Y} \nabla p-f \\
-e^{Y} \nabla p \cdot \mathbf{n}-B_{N} \\
p(x, 0)-I(x)
\end{array}\right]
$$

where $\mathcal{Y}$ will be defined below. As we indicated earlier this operator (see equation (3.1)) has important applications in subsurface flow modeling. Another interesting example can be obtained if $e^{Y}$ is the hydraulic conductivity of an aquifer. Then, $G(Y, p)=0$ may take the form of the PDE system that describes the groundwater flow. Therefore, the inverse estimation of $Y$ by means of data assimilation is of great relevance for the subsurface community. For this reason we have chosen these problems in order to validate the IRBS. First, we need to verify that the operator $G$ satisfies the hypothesis required to apply IRBS. In the next sections we discuss some numerical results relevant to the applications mentioned above. 


\subsection{An application of the abstract framework}

Assume $\Omega \subset \mathbb{R}^{N}$ has the strong Lipschitz property. Let $C_{Y}$ be a covariance operator such that

$$
\|Y\|_{\mathcal{Y}}^{2} \equiv \int_{\Omega} \int_{\Omega} Y C_{Y}^{-1} Y
$$

is equivalent to the Sobolev norm $H^{3}(\Omega)$ for $N=2,3$. Then, from the Sobolev imbedding theorem, $H^{3}(\Omega) \hookrightarrow C^{1}(\bar{\Omega})$. Let us define the space $\mathcal{Y}$ to be the completion of $C^{1}(\bar{\Omega})$ with the norm $\|\cdot\|_{\mathcal{Y}}$. Then, (A1) is satisfied. Given $H_{m} \in L^{2}\left(0, T ; L^{2}(\Omega)\right)$, for $m=1, \cdot, M$, we define the measurement functional by

$$
\mathcal{L}_{m}(p)=\int_{0}^{T} \int_{\Omega} H_{m}(x, t) p(x, t) d x d t
$$

From standard PDEs theory, we have the following theorem

Theorem 4. For each $Y \in \mathcal{Y} \hookrightarrow C^{1}(\bar{\Omega})$, and $\left(f, B_{N}, I\right) \in L^{2}\left(0, T ; L^{2}(\Omega)\right) \times H^{1 / 2,1 / 4}\left(\Gamma_{T}\right) \times$ $H^{1}(\Omega)$, there exists a unique $p \in H^{2,1}\left(\Omega_{T}\right)$ such that $G(Y, p)=0$ and

$$
\|p\|_{H^{2,1}\left(\Omega_{T}\right)} \leq C\left[\|f\|_{L^{2}\left((0, T) ; L^{2}(\Omega)\right)}+\left\|B_{N}\right\|_{H^{1 / 2,1 / 4}\left(\Gamma_{T}\right)}+\|I\|_{H^{1}(\Omega)}\right]
$$

where $C$ is constant that only depends on an upper bound for $\|Y\|_{\mathcal{Y}}$, a lower bound for $e^{Y}$, $\Omega$ and $T$.

\section{Proof: See [46].}

From this result we deduce the following corollary:

Corollary 1. Let $\left(f, B_{N}, I\right) \in L^{2}\left(0, T ; L^{2}(\Omega)\right) \times H^{1 / 2,1 / 4}\left(\Gamma_{T}\right) \times H^{1}(\Omega)$ be fixed. Take $\hat{Y} \in Y$ arbitrary and $r>0$. Consider $B(r, \hat{Y})$ in $\mathcal{Y}$. Then, there exists a constant $C^{*}>0$ such that for every $Y \in B(r, \hat{Y})$, the solution to the following problem

$$
\begin{array}{r}
\frac{\partial h}{\partial t}-\nabla \cdot e^{Y} \nabla h=f \\
\nabla h \cdot \mathbf{n}=B_{N} \\
h(x, 0)=I
\end{array}
$$

satisfies

$$
\|h\|_{H^{2,1}\left(\Omega_{T}\right)} \leq C^{*}\left[\|f\|_{L^{2}\left((0, T) ; L^{2}(\Omega)\right)}+\left\|B_{N}\right\|_{H^{1 / 2,1 / 4}\left(\Gamma_{T}\right)}+\|I\|_{H^{1}(\Omega)}\right]
$$


$C^{*}$ depends only on $T, \Omega, \hat{Y}$ and $r$. In addition, for any $Y \in B(r, \hat{Y})$ we have that

$$
\left\|e^{Y}\right\|_{C^{1}(\bar{\Omega})} \leq \omega(\hat{Y}, r)
$$

where $\omega$ is a constant that depends only on $\hat{Y}$ and $r$.

Proof: For all $Y \in B(r, \hat{Y})$, we have that $\|Y-\hat{Y}\|_{\mathcal{Y}} \leq r$. By the Sobolev imbedding we know that

$$
\|Y-\hat{Y}\|_{C^{1}(\bar{\Omega})} \leq r
$$

Trivially, $|Y(x)-\hat{Y}(x)| \leq r$ for all $x \in \Omega$. Then

$$
Y_{*} \equiv-r+\min _{\bar{\Omega}} \hat{Y} \leq-r+\hat{Y}(x) \leq Y(x) \leq r+\hat{Y}(x) \leq r+\max _{\bar{\Omega}} \hat{Y} \equiv Y^{*} .
$$

A similar argument shows

$$
D Y_{*} \equiv-r+\min _{\bar{\Omega}} \hat{Y}^{\prime} \leq-r+\hat{Y}^{\prime}(x) \leq Y^{\prime}(x) \leq r+\hat{Y}^{\prime}(x) \leq r+\max _{\bar{\Omega}} \hat{Y}^{\prime} \equiv D Y^{*}
$$

Note that the minimum and maximum above exist since $\hat{Y}$ and $\hat{Y}^{\prime}$ are continuous and defined on $\bar{\Omega}$ which is compact. Then $Y_{*} \leq Y(x) \leq Y^{*}$ for all $x \in \bar{\Omega}$ and so

$$
0<e^{Y_{*}} \leq e^{Y(x)} \leq e^{Y^{*}}
$$

for all $x \in \bar{\Omega}$ and for all $Y \in B$. In addition, from (4.10) we have that $\|Y\|_{C^{1}(\bar{\Omega})} \leq$ $r+\|\hat{Y}\|_{C^{1}(\bar{\Omega})}$. Therefore for every $Y \in B(r, \hat{Y})$ we use $r+\|\hat{Y}\|_{C^{1}(\bar{\Omega})}$ as an upper bound and $e^{Y_{*}}$ as a lower bound in Theorem 4 to find

$$
\|p\|_{H^{2,1}\left(\Omega_{T}\right)} \leq C^{*}\left[\|f\|_{L^{2}\left((0, T) ; L^{2}(\Omega)\right)}+\left\|B_{N}\right\|_{H^{1 / 2,1 / 4}\left(\Gamma_{T}\right)}+\|I\|_{H^{1}(\Omega)}\right] .
$$

Finally from (4.13) and (4.12), $D Y_{*} e^{Y_{*}} \leq Y^{\prime}(x) e^{Y(x)} \leq D Y^{*} e^{Y^{*}}$. Thus, $\left|Y^{\prime}(x) e^{Y(x)}\right| \leq$ $\min \left\{-D Y_{*} e^{Y_{*}}, D Y^{*} e^{Y^{*}}\right\}$ from which it follows

$$
\left\|e^{Y}\right\|_{C^{1}(\bar{\Omega})}=\max _{\bar{\Omega}}\left[\left|e^{Y(x)}\right|+\left|Y^{\prime}(x) e^{Y(x)}\right|\right] \leq e^{Y^{*}}+\min \left\{-D Y_{*} e^{Y_{*}}, D Y^{*} e^{Y^{*}}\right\} \equiv \omega(r, \hat{Y})
$$

Proposition 1. The operator $G$ defined by (4.1) satisfies conditions (F1)-(F3). 


\section{Proof:}

(F1) $G$ is continuously Frechet-differentiable. This results can be shown easily and so the proof is omitted. It can actually be shown that

$$
D G(\tilde{Y}, \tilde{p})[\delta Y, \delta p]=D_{Y} G(\tilde{Y}, \tilde{p}) \delta Y+D_{p} G(\tilde{Y}, \tilde{p}) \delta p
$$

where

$$
D_{Y} G(\tilde{Y}, \tilde{p}) \delta Y=\left[\begin{array}{c}
-\nabla \cdot e^{\tilde{Y}} \delta Y \nabla \tilde{p} \\
-e^{\tilde{Y}} \delta Y \nabla \tilde{p} \cdot \mathbf{n} \\
0
\end{array}\right], \quad D_{p} G(\tilde{Y}, \tilde{p}) \delta p=\left[\begin{array}{c}
\frac{\partial \delta p}{\partial t}-\nabla \cdot e^{\tilde{Y}} \nabla \delta p \\
-e^{\tilde{Y}} \nabla \delta p \cdot \mathbf{n} \\
\delta p(x, 0)
\end{array}\right]
$$

(F2) This is exactly Theorem 4.

(F3) From (4.17) and Theorem 4 it follows that, for any $\left(f, B_{C}, I\right) \in L^{2}\left(0, T ; L^{2}(\Omega)\right) \times$ $H^{1 / 2,1 / 4}\left(\Gamma_{T}\right) \times H^{1}(\Omega)$, problem

$$
\left[\begin{array}{c}
\frac{\partial \delta p}{\partial t}-\nabla \cdot e^{\tilde{Y}} \nabla \delta p \\
-e^{\tilde{Y}} \nabla \delta p \cdot \mathbf{n} \\
\delta p(x, 0)
\end{array}\right]=\left[\begin{array}{c}
f \\
B_{N} \\
I
\end{array}\right]
$$

has a unique solution $\delta p$. Then, $D_{p} G(\tilde{Y}, \tilde{p}) \delta p=\left(f, B_{C}, I\right)$ which proves surjection. Uniqueness implies injectivity and so the isomorphism. Let us now consider any $B(r, \hat{Y})$. We realize that (3.3) is equivalent to $D_{Y} G(Y, p) \delta Y+D_{p} G(Y, p) \delta p=0$ which implies

$$
\begin{array}{rcc}
\frac{\partial \delta p}{\partial t}-\nabla \cdot e^{Y} \nabla \delta p= & \nabla \cdot e^{Y} \delta Y \nabla p \\
e^{Y} \nabla \delta p \cdot \mathbf{n}= & -e^{Y} \delta Y \nabla p \cdot \mathbf{n} \\
\delta p(x, 0)= & 0 .
\end{array}
$$

Then, for $Y \in B(r, \hat{Y})$, we apply (4.8) to find that

$$
\begin{aligned}
&\|\delta p\|_{H^{2,1}\left(\Omega_{T}\right)} \leq C^{*}(\| \nabla\left.\cdot e^{Y} \delta Y \nabla p\left\|_{L^{2}\left((0, T) ; L^{2}(\Omega)\right)}+\right\| e^{Y} \delta Y \nabla p \cdot \mathbf{n} \|_{H^{1 / 2,1 / 4}\left(\Gamma_{T}\right)}\right) \\
& \leq C^{*}\|\delta Y\|_{\mathcal{Y}}\left[\left\|e^{Y}\right\| \mathcal{Y}\|p\|_{H^{2,1}\left(\Omega_{T}\right)}+\left\|B_{N}\right\|_{H^{1 / 2,1 / 4}\left(\Gamma_{T}\right)}\right]
\end{aligned}
$$


From (4.8) and (4.9)

$$
\begin{array}{r}
\|\delta p\|_{H^{2,1}\left(\Omega_{T}\right)} \leq C^{*}\|\delta Y\| \mathcal{Y}\left[\omega ( r , \hat { Y } ) C ^ { * } \left[\|f\|_{L^{2}\left((0, T) ; L^{2}(\Omega)\right)}\right.\right. \\
\left.\left.+\left\|B_{N}\right\|_{H^{1 / 2,1 / 4}\left(\Gamma_{T}\right)}+\|I\|_{H^{1}(\Omega)}\right]+\left\|B_{N}\right\|_{H^{1 / 2,1 / 4}\left(\Gamma_{T}\right)}\right]
\end{array}
$$

from which it follows that $\|\delta p\|_{H^{2,1}\left(\Omega_{T}\right)} \leq C_{1}\|\delta Y\| \mathcal{Y}$ for

$$
\begin{array}{r}
C_{1}=C^{*}\left[\omega(r, \hat{Y}) C^{*}\left[\|f\|_{L^{2}\left((0, T) ; L^{2}(\Omega)\right)}+\left\|B_{N}\right\|_{H^{1 / 2,1 / 4}\left(\Gamma_{T}\right)}+\|I\|_{H^{1}(\Omega)}\right]\right. \\
\left.+\left\|B_{N}\right\|_{H^{1 / 2,1 / 4}\left(\Gamma_{T}\right)}\right]
\end{array}
$$

and so (F3) is proved.

The convergence results of the IRBS applied to problem (4.1) and (4.3) can be summarized in the following Corollary.

Corollary 2. Assume that the hypothesis of Proposition 1 as well as (A2) hold. Given $\tau>1$, if

$$
\theta \equiv C_{1}^{2}\|\mathcal{L}\|^{2}\left\|C^{-1}\right\|<\tau-1
$$

and the initial guess (prior knowledge) $\bar{k}$ is close enough to a solution to the NF-PIP, then the IRBS converges to an approximate solution in the sense of Definition 1.

Proof. Since (A1)-(A2), (F1)-(F3) are satisfied, we can apply the results of Chapter 3.

Remark 3. From the definition of $C_{1}$ (4.24) we point out that (4.25) balances relative magnitudes of the assimilation information and the forward model. More precisely, for a fixed ball $B(r, \hat{Y}), \theta$ is a function of $\hat{Y}, f, I, B_{C}, e^{\hat{Y}}, \Omega, T, \mathcal{L}, C$. In practice, (4.25) may be difficult or impossible to verify. Nevertheless, in Section 6 the numerical experiments show that, for example, for different values $\tau>1$, the result of Corollary 2 holds.

Remark 4. Note that we have assumed that (A2) holds in Corollary 2. Proving Assumption (A2) for (4.1) and (4.3) is still an open problem. However, our numerical results show that convergence is achieved for a broad class of experiments.

It is worth mentioning that Hanke in [32] proved Assumption (A2) for $G$ given by (4.1) but for $K=e^{Y}$. He also considered the identity mapping as the measurement operator. In that case, the operator $G(K, p)$ is bilinear and the proofs can be obtained by standard PDE techniques. 


\subsection{Implementation}

For the implementation of the IRBS we need the explicit form of $R^{n}$ as well as $\gamma^{n}$. To obtain closed form relations, we first recall that $k^{n}$ (3.21) was derived from the first order optimality condition on $J^{n}$. For the application under consideration, this can be obtained by considering the Lagrangian:

$$
\begin{array}{r}
\mathcal{Q}^{n}[Y, p, \lambda]=\frac{1}{2}[d-\mathcal{L}(p)]^{T} C^{-1}[d-\mathcal{L}(p)] \\
+\int_{\Omega} \int_{\Omega}\left(Y(x)-Y^{n-1}(x)\right) C_{Y}^{-1}\left(x, x^{\prime}\right)\left(Y\left(x^{\prime}\right)-Y^{n-1}\left(x^{\prime}\right)\right)+\int_{\Omega} \int_{0}^{T} \lambda L G(p, Y)
\end{array}
$$

where

$$
L G(p, Y) \equiv\left[\begin{array}{c}
\frac{\partial p}{\partial t}-\nabla \cdot\left(e^{Y^{n-1}} \nabla p\right)-\nabla \cdot\left(Y-Y^{n-1}\right) e^{Y^{n-1}} \nabla p_{F}^{n}-f \\
\left.\left(e^{Y^{n-1}} \nabla p \cdot \mathbf{n}+\left(Y-Y^{n-1}\right) e^{Y^{n-1}} \nabla p_{F}^{n} \cdot \mathbf{n}\right)\right|_{\Gamma_{N}}-B_{N} \\
\left.p\right|_{t=0}-I
\end{array}\right]=0
$$

is the linearized constraint. Then, the first order optimality condition $D \mathcal{Q}^{n}[Y, p, \lambda]=0$ gives rise to the following Euler-Lagrange (E-L) system.

State:

$$
\begin{array}{rlrl}
\frac{\partial p^{n}}{\partial t}-\nabla \cdot\left(e^{Y^{n-1}} \nabla p^{n}\right)-\nabla \cdot\left(Y^{n}-Y^{n-1}\right) e^{Y^{n-1}} \nabla p_{F}^{n} & = & f & \text { on } \Omega \times(0, T] \\
e^{Y^{n-1}} \nabla p^{n} \cdot \mathbf{n}+\left(Y^{n}-Y^{n-1}\right) e^{Y^{n-1}} \nabla p_{F}^{n} \cdot \mathbf{n}= & B_{N} & \text { on } \Gamma_{N} \times(0, T], \\
p^{n}= & I, & & \text { in } \Omega \times\{0\}
\end{array}
$$

\section{Adjoint:}

$$
\begin{aligned}
& -\frac{\partial \lambda^{n}}{\partial t}-\nabla \cdot\left(e^{Y^{n-1}} \nabla \lambda^{n}\right)=\left(d-\mathcal{L}\left(p^{n}\right)\right) C^{-1} H \quad \text { in } \Omega \times(0, T], \\
& e^{Y^{n-1}} \nabla \lambda^{n} \cdot \mathbf{n}=\quad 0 \quad \text { on } \Gamma_{N} \times(0, T], \\
& \lambda^{n}=\quad 0 \quad \text { in } \Omega \times\{T\},
\end{aligned}
$$

\section{Parameter:}

$$
Y^{n}(x)=Y^{n-1}(x)-\int_{0}^{T} \int_{\Omega} C_{Y}\left(x, x^{\prime}\right) \nabla \lambda^{n}\left(x^{\prime}, t\right) \cdot e^{Y^{n-1}\left(x^{\prime}\right)} \nabla p_{F}^{n}\left(x^{\prime}, t\right) d t d x^{\prime} .
$$


Here, $H=\left[H_{1}, \ldots, H_{M}\right]$ and $p_{F}^{n}$ is the solution to $G\left(Y^{n-1}, p_{F}^{n}\right)=0$ (with $G$ from (4.1)). The representers can be obtained by postulating that

$$
p^{n}=p_{F}^{n}+\sum_{m=1}^{M} \beta_{m}^{n} r_{m}^{n}, \quad \lambda^{n}=\sum_{m=1}^{M} \beta_{m}^{n} \alpha_{m}^{n}, \quad Y^{n}=Y^{n-1}+\sum_{m=1}^{M} \beta_{m}^{n} \gamma_{m}^{n}
$$

for some functions $r_{m}^{n}(x, t), \alpha_{m}^{n}(x, t)$ and $\gamma_{m}^{n}(x)$ and scalars $\beta_{m}^{n}$ to be determined. When the previous expressions are substituted in the E-L system, we obtain that (4.35) solves the E-L system if and only if $r_{m}^{n}$ is the solution to

$$
\begin{array}{rlrl}
\frac{\partial r_{m}^{n}}{\partial t}-\nabla \cdot\left(e^{Y^{n-1}} \nabla r_{m}^{n}\right)-\nabla \cdot\left(\gamma_{m}^{n} e^{Y^{n-1}} \nabla p_{F}^{n}\right) & =0, & & \text { in } \Omega \times(0, T], \\
e^{Y^{n-1}} \nabla r_{m}^{n} \cdot \mathbf{n}+\gamma_{m}^{n} e^{Y^{n-1}} \nabla p_{F}^{n} \cdot \mathbf{n} & =0 & & \text { on } \Gamma \times(0, T], \\
r_{m}^{n} & =0, & \text { in } \Omega \times\{0\},
\end{array}
$$

$\alpha_{m}^{n}$ is the solution to

$$
\begin{aligned}
-\frac{\partial \alpha_{m}^{n}}{\partial t}-\nabla \cdot\left(e^{Y^{n-1}} \nabla \alpha_{m}^{n}\right) & =H_{m} & & \text { in } \Omega \times(0, T], \\
e^{Y^{n-1}} \nabla \alpha_{m}^{n} \cdot \mathbf{n} & =0 & & \text { on } \Gamma_{N} \times(0, T], \\
\alpha_{m}^{n} & =0 & & \text { in } \Omega \times\{T\},
\end{aligned}
$$

and $\gamma_{m}^{n}$ satisfies

$$
\gamma_{m}^{n}(x)=-\int_{0}^{T} \int_{\Omega} C_{Y}\left(x, x^{\prime}\right) \nabla \alpha_{m}^{n}\left(x^{\prime}, t\right) \cdot e^{Y^{n-1}\left(x^{\prime}\right)} \nabla p_{F}^{n}\left(x^{\prime}, t\right) d t d x^{\prime} .
$$

and the representer coefficients are given by (3.28) as

$$
\left(R^{n}+C\right) \beta^{n}=d-\mathcal{L}\left(p_{F}^{n}\right)
$$

with the matrix $R^{n}$ defined by $R_{i j}^{n}=\mathcal{L}_{i}\left(r_{j}^{n}\right)$. For this problem, we have directly derived the representer method in [36].

We now prove the following

Proposition 2. The representers are given by expression (4.42).

Proof: Let us take $\delta Y \in \mathcal{Y}$ arbitrary and consider $\delta p$ the solution to $L G(\delta p, \delta Y)=0$. We want to prove (see Section (3.3.2) ) that for every $m \in\{1, \ldots, M\},\left\langle\gamma_{m}, \delta Y\right\rangle_{\mathcal{Y}}=\mathcal{L}_{m}(\delta p)$. Multiplying (4.39) by $\delta p$, integrating by parts and using (4.41)-(4.40) as well as the definition 
of $\mathcal{L}_{m}$ yields

$$
\begin{aligned}
\int_{\Omega}\left(\alpha_{m}^{n}(t=0) \delta p(t=0)\right) & +\int_{\Omega} \int_{0}^{T}\left[\frac{\partial \delta p}{\partial t}-\nabla \cdot\left(e^{Y^{n-1}} \nabla \delta p\right)\right] \alpha_{m}^{n} \\
& +\int_{\Gamma} \int_{0}^{T} e^{Y^{n-1}} \nabla \delta p \cdot \mathbf{n} \alpha_{m}^{n}=\mathcal{L}_{m}(\delta p),
\end{aligned}
$$

and from (4.27) we find

$$
\int_{\Omega} \int_{0}^{T} \nabla \cdot\left(\delta Y e^{Y^{n-1}} \nabla p_{F}^{n}\right) \alpha_{m}^{n}-\int_{\Gamma} \int_{0}^{T} \delta Y e^{Y^{n-1}} \nabla p_{F}^{n} \cdot \mathbf{n} \alpha_{m}^{n}=\mathcal{L}_{m}(\delta p) .
$$

Integrating by parts again yields

$$
-\int_{\Omega} \int_{0}^{T} \delta Y e^{Y^{n-1}} \nabla p_{F}^{n} \cdot \nabla \alpha_{m}^{n}=\mathcal{L}_{m}(\delta p) .
$$

On the other hand, we multiply (4.42) by $C_{Y}^{-1} \delta Y$, integrate and use (3.6) to find

$$
\int_{\Omega} \int_{\Omega} \gamma_{m}^{n} C_{Y}^{-1} \delta Y=-\int_{\Omega} \int_{0}^{T} \nabla \alpha_{m}^{n} \cdot e^{Y^{n-1}} \nabla p_{F}^{n} \delta Y
$$

Thus, combining (4.46) and (4.47) we obtain

$$
\left\langle\gamma_{m}, \delta Y\right\rangle_{\mathcal{Y}} \equiv \int_{\Omega} \int_{\Omega} \gamma_{m}^{n} C_{Y}^{-1} \delta Y=\mathcal{L}_{m}(\delta p)
$$

for all $\delta Y$ and $\delta p$ that satisfy (4.27). Therefore, since the representer obtained by the Riez theorem is unique, we have proved the result

In this section we present some numerical examples to show the capabilities of the IRBS. First, some aspects related to the implementation are discussed.

\subsubsection{Discretization}

Note from the previous section that systems (4.31)-(4.40) need to be solved at each iteration. For the spatial discretization, a cell-centered finite differences (CCFD) scheme is utilized [3]. Time discretization is implemented with a Backward-Euler scheme. The coefficient of the second order elliptic operator is assumed to be constant at each cell. Then, with the CCFD discretization, the coefficient in each cell edge is approximated by the harmonic average. For the present analysis we use covariance functions for which (4.42) becomes a convolution which can be computed efficiently by the FFT. 


\subsubsection{Measurement Functional}

For each measurement location and time, we assume that the measurement functional is the average of the state variable, over a small cube around the measurement and time location. In other words, $\mathcal{L}_{m}$ is given by (4.3) with

$$
\begin{gathered}
H_{m}(x, y, t)=\left[\begin{array}{cc}
1 & \text { if }(x, y) \in \mathcal{R} t-t_{m} \leq s_{t} \\
0 & \text { else }
\end{array}\right] \\
\mathcal{R}=\left\{(x, y):\left|x-x_{m}\right| \leq s_{x},\left|y-y_{m}\right| \leq s_{y}\right\}
\end{gathered}
$$

where $s_{x}$ and $s_{y}$ are defined so that $R$ coincides with the cell containing $\left(x_{m}, y_{m}\right)$ and $s_{t}$ is defined so that $\left[t_{m}, t_{m}+s_{t}\right)$ corresponds to the time interval containing $t_{m}$. This definition serves as an approximation to pointwise measurements.

\subsubsection{Prior Error Information and Norms}

As indicated before, Assumption (A1) is an essential assumption for the previous analysis. More precisely, we need an equivalence between the norm induced by the inverse covariance operator and a Sobolev norm. Some equivalences of this type can be found in $[53,70,65]$ for different types of covariance functions. However, the assumptions on the regularity of the coefficient of the second order elliptic operator may not be preserved by the discretization scheme. In addition, direct computation of (4.2) may become computationally prohibited and so it is avoided in these experiments. Moreover, as we pointed out before, the permeability is assumed to be piecewise constant. For this reason we relax the assumptions on the regularity for the parameter and the $L^{2}(\Omega)$-norm ( $l^{2}$ norm for the discretized problem) is considered for convergence analysis in the parameter space. Additionally, we need to specify the prior error covariance $C_{Y}$, and the prior mean $\bar{Y}$ which is used as the first guess $\left(Y^{0}=\bar{Y}\right)$ in the IRBS. For the following examples, the experimental omnidirectional semivariogram [17] of the true parameter is computed and a variogram model is fitted. Then, $C_{Y}$ is defined as the covariance of the associated variogram model of the true permeability.

\subsubsection{Generation of Synthetic Data}

For the following set of experiments, a "true permeability" ("true hydraulic conductivity") is prescribed. Then, we generate synthetic data $d^{\eta}$ by running the model simulator with the "true parameter" and applying the measurement functional defined by (4.3) with (4.49). In order to avoid an "inverse crime", this data is obtained from a finer grid than the one 
utilized for the inverse computations. Additionally, Gaussian noise with zero mean and covariance $C=\sigma_{\eta}^{2} I_{M \times M}$ is added to the measurement. In our choice of $C, I_{M \times M}$ is the $M \times M$ identity matrix where $M$ is the number of measurements for the corresponding experiment. The true parameter is used only to compare to our final estimated parameter.

\subsubsection{Stopping rule}

The stopping criteria for the algorithm presented in Section IRBS requires an estimate of the noise level. However, only the measurements $\left(d^{\eta}\right)$ as well as the prior error statistics $\left(\sigma_{\eta}\right)$ may be available. To obtain an estimate of the error level in the case $C=\sigma_{\eta}^{2} I$, we assume that $d^{\eta}=d+\sigma_{\eta} e$ where $e$ is a Gaussian random variable with zero mean and variance equal one. Therefore, since $d^{\eta}-d=\sigma_{\eta} e$, if we assume that $\left|e_{j}\right| \leq 1$, it follows $\sum_{j}^{M}\left|d_{j}^{\eta}-d_{j}\right|^{2}=\sigma_{\eta}^{2} \sum_{j}^{M}\left|e_{j}\right|^{2} \leq \sigma_{\eta}^{2} M$. Thus, $\left\|d^{\eta}-d\right\| \leq \sigma_{\eta} \sqrt{M}$. With this argument we propose to use $\eta=\sigma_{\eta} \sqrt{M}$ as the noise level for the algorithm. For the stopping criteria, we recall that the IRBS is terminated when $\left\|d^{\eta}-\mathcal{L}\left(s_{F}^{n}\right)\right\| \leq \tau \sigma_{\eta} \sqrt{M}$ for the first time. Having discussed some general considerations, we now introduce some examples. The objective of these experiments is to analyze the efficiency of the IRBS to obtain improved parameters while fitting the synthetic data.

\subsubsection{The Groundwater Flow}

Groundwater flow can be modeled by the following equation [8]

$$
S \frac{\partial h}{\partial t}-\nabla \cdot e^{Y} \nabla h=f
$$

where $S$ is specific storage, $f$ accounts for sources, $e^{Y}$ is the hydraulic conductivity and $h$ is the piezometric head. When proper boundary conditions are imposed, (4.51) takes the form (up to known constants) of the operator (4.1). The parameter to be estimated is $Y$ and all the other parameters are assumed to be perfectly known.

\section{Experiment I.}

In order to validate the IRBS presented in the previous sections, we consider the same problem used in [32]. The forward model is the steady state of (4.51) over a square domain 
of dimension $6 \mathrm{~km} \times 6 \mathrm{~km}$. The boundary conditions are given by,

$$
\begin{aligned}
u(x, 0)=100 \mathrm{~m}, & u_{x}(6 \mathrm{~km}, y)=0, \\
-e^{Y} u_{x}(0, y)=500 \mathrm{~m}^{3} \mathrm{~km}^{-1} \mathrm{day}^{-1}, & u_{y}(x, 6 \mathrm{~km})=0
\end{aligned}
$$

and a constant (in time) recharge is represented by

$$
f(x, y)=\left\{\begin{array}{cc}
0 & 0<y<4 \mathrm{~km} \\
0.137 \times 10^{-3} \mathrm{~m} / \text { day } & 4 \mathrm{~km}<y<5 \mathrm{~km} \\
0.274 \times 10^{-3} \mathrm{~m} / \text { day } & 5 \mathrm{~km}<y<6 \mathrm{~km}
\end{array}\right.
$$

The grid used for the synthetic data generation and for the IRBS are $180 \times 180$ and $60 \times 60$ respectively. The measurement locations are shown in Figure 4.1(a). For the prior mean of $Y=\ln K$ we select the natural logarithm of the initial guess used in [32]. Note from Figure 4.1 (b), that the true conductivity is geometrically anisotropic and therefore, the omnidirectional semivariogram is poor prior knowledge of the truth. It is the purpose of this example to illustrate the efficiency of our method when limited information is provided. The covariance associated with the variogram model fitted to the experimental semivariogram as well as other pertinent information are displayed in Table 4.1. In Experiment IA and IB we follow [32] and test the scheme for two different signals-to-noise ratios (SNR). The estimated results are displayed in Figure 4.1 (c)(d) where a good agreement to the true log hydraulic conductivity is obtained. Figure 4.1(e) shows the true and estimated hydraulic conductivity fields projected along the phantom shown in Figure 4.1(a) (dotted red line). Convergence history for IA and IB are displayed in Table 4.2. Note that for smaller (SNR), the IRBS provides smaller error in the computed parameter. In contrast to the work in [32] where the hydraulic conductivity $K$ is identified, in our approach the estimation is performed for $Y=\ln K$. Although the latter approach imposes a stronger nonlinearity in the abstract formulation, the implementation does not require modification when negative values of the parameter occur. This may become an advantage in problems (see Experiment III) where the true parameter changes by several orders of magnitude, and further modifications may be detrimental to the quality of the identification.

We remark that the measurement data is assimilated only from the measurement locations. Unlike the standard parameter identification approaches, there is no need to interpolate the entire field of the state variable. Moreover, [32] reported that, when the parameter identification problem is posed with a finite-dimensional measurement space, their method becomes considerably less efficient. On the other hand, the IRBS convergence is 
TABLE 4.1: Information for data assimilation. Experiment I.

\begin{tabular}{lll}
\hline Experiment & IA & IB \\
$\#$ of Measurement Locations & 18 & 18 \\
$\tau$ & 1.5 & 1.5 \\
$\eta \tau[\mathrm{m}]$ & 11.4551 & 1.1455 \\
$\sigma_{\eta}[\mathrm{m}]$ & 1.8 & 0.18 \\
$\bar{Y}[\ln \mathrm{m} /$ day $]$ & $\ln 20$ & $\ln 20$ \\
$C_{Y}\left(\xi, \xi^{\prime}\right)[\ln (\mathrm{m} / \text { day })]^{2}$ & $2.2 \exp \left[-\frac{\left\|\xi-\xi^{\prime}\right\|}{1 \mathrm{~km}}\right]$ & $2.2 \exp \left[-\frac{\left\|\xi-\xi^{\prime}\right\|}{1 \mathrm{~km}}\right]$ \\
signal-to-noise ratio (SNR) & 102.27 & 1034 \\
\hline
\end{tabular}

achieved in a few iterations with satisfactory results. Such behavior should not be surprising since the present approach takes into account prior information.

TABLE 4.2: Numerical Results. Experiment I.

\begin{tabular}{lllll}
\hline & Experiment IA & & Experiment IB & \\
$n$ & $\left\|Y^{n-1}-Y_{\text {true }}\right\|_{l^{2}}$ & $\left\|d^{\eta}-\mathcal{L}\left(p_{F}^{n}\right)\right\|$ & $\left\|Y^{n-1}-Y_{\text {true }}\right\|_{l^{2}}$ & $\left\|d^{\eta}-\mathcal{L}\left(p_{F}^{n}\right)\right\|$ \\
1 & 178.9116 & 409.9119 & 178.9116 & 410.4575 \\
2 & 114.0224 & 125.5916 & 116.4194 & 124.7171 \\
3 & 81.7970 & 27.4934 & 83.2218 & 24.8984 \\
4 & 70.4071 & 3.7471 & 69.2700 & 2.4368 \\
5 & - & - & 67.8318 & 0.0685 \\
\hline
\end{tabular}

\subsubsection{Single-phase Darcy flow through porous media}

We now study the reservoir model described by (3.1).

\section{Experiment II.}

In Experiment II we are interested in determining the efficiency for the estimation of the log permeability $Y=\ln K$ from pressure data collected at different configurations. The true log permeability (Figure 4.2 (a)) is generated stochastically with the routine SGSIM [17]. Table 4.3 shows the reservoir properties and Table 4.4 presents prior error information. Table 4.5 provides the corresponding information for each run whose corresponding estimations are presented in 4.2 (b)-(f). For experiments IIB, IIC and IID, the observation wells are equally spaced over the domain but they do not coincide with either the injection or the 


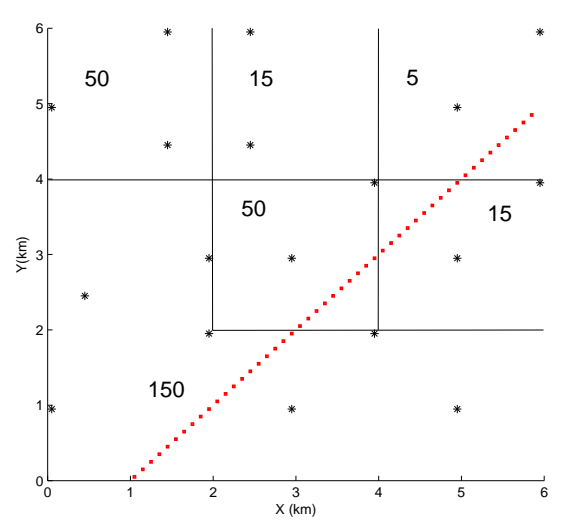

(A) Configuration

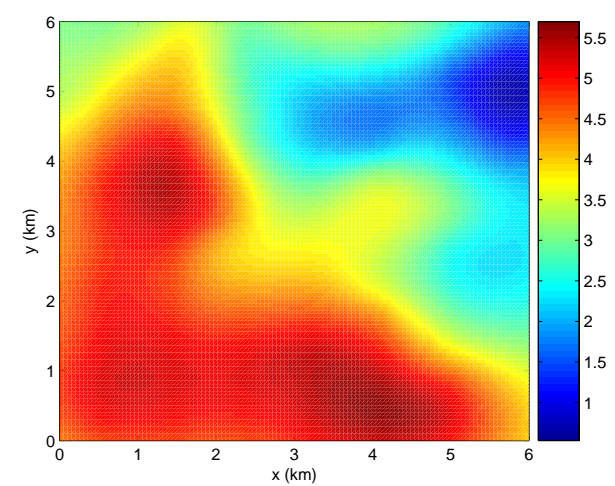

(c) Experiment $I A$

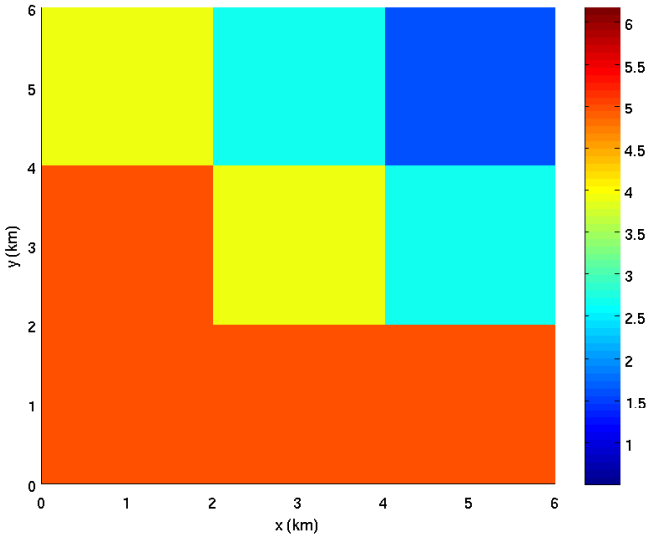

(B) True

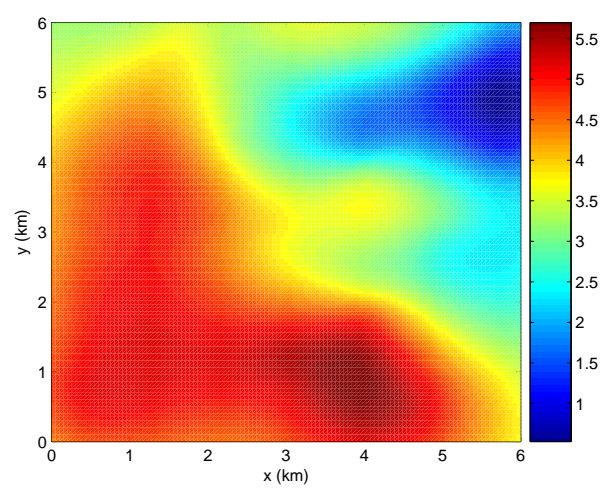

(D) Experiment IB

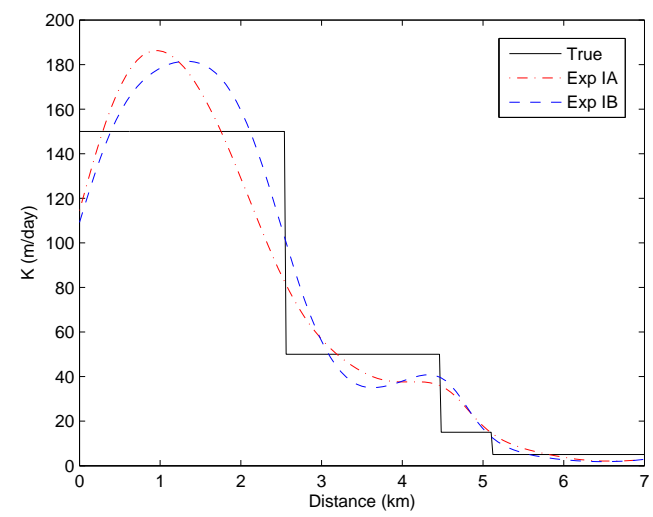

(E) Phantom

Figure 4.1: Experiment I. Top Left: Aquifer Configuration (observation wells are indicated by *). Top Right and Middle: log hydraulic conductivity fields $[\mathrm{ln}(\mathrm{m} /$ days $)]$. Bottom: Hydraulic conductivity [m/days] fields projected over the phantom 
production wells. For experiments IIA and IIE measurements are collected at the injection and production wells. It comes as no surprise that the quality of the reconstruction depends on the number of measurements available. However, the measurement locations also affects the final outcome. It is also worth mentioning that the best estimate of log permeability was obtained with Experiment IIE which corresponds to the configuration of maximum number of observation wells. Additionally, convergence is achieved within the first five iterations for all the different configurations. Clearly, non uniqueness is always evident since for a small number of observations, there are many possible parameters that will fit the data

\begin{tabular}{lcc} 
TABLE 4.3: Reservoir description. & Experiment II and Experiment III. \\
\hline Experiment & II & III \\
Dimension $\mathrm{ft}^{3}$ & $2000 \times 2000 \times 2$ & $2000 \times 4000 \times 2$ \\
Grid Blocks (for synthetic data) & $180 \times 180 \times 1$ & $60 \times 220 \times 1$ \\
Grid Blocks (for IRBS) & $60 \times 60 \times 1$ & $60 \times 110 \times 1$ \\
Time interval $[0, T]$ (days) & {$[0,1]$} & {$[0,1]$} \\
Time discretization $d t$ (days) & 0.01 & 0.01 \\
Porosity & 0.2 & 0.2 \\
Water compressibility (psi ${ }^{-1}$ ) & $3.1 \mathrm{e}-6$ & $3.1 \mathrm{e}-6$ \\
Rock compressibility (psi ${ }^{-1}$ ) & $1.0 \mathrm{e}-6$ & $1.0 \mathrm{e}-6$ \\
Water viscosity (cp) & 0.3 & 0.3 \\
Initial pressure (psi) & 5000 & 10000 \\
Number Injection Wells & 2 & 4 \\
Number Production Wells & 6 & 5 \\
Shut-in Time $t_{s}$ (day) & 0.75 & 0.75 \\
a Injection rate (stb/day) & 2500 & 1000 \\
${ }^{a}$ Production rate (stb/day) & 833.33 & 1000 \\
\hline
\end{tabular}

a Rate per well in the interval $\left[0, t_{s}\right]$.

TABLE 4.4: Prior error information for Experiment II.

\begin{tabular}{ll}
$\tau$ & 2.0 \\
$\sigma_{\eta}[\mathrm{psi}]$ & 5.0 \\
$\bar{Y}[\ln \mathrm{md}]$ & 196.42 \\
$C_{Y}\left(\xi, \xi^{\prime}\right)[\ln (\mathrm{md})]^{2}$ & $0.95 \exp \left[-\frac{\left\|\xi-\xi^{\prime}\right\|}{400 \mathrm{ft}}\right]$ \\
$\sigma_{\eta}[\mathrm{psi}]$ & 5.0 \\
\hline
\end{tabular}




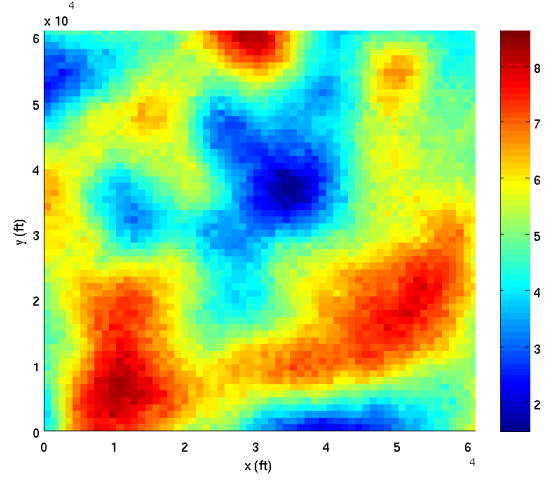

(A) True

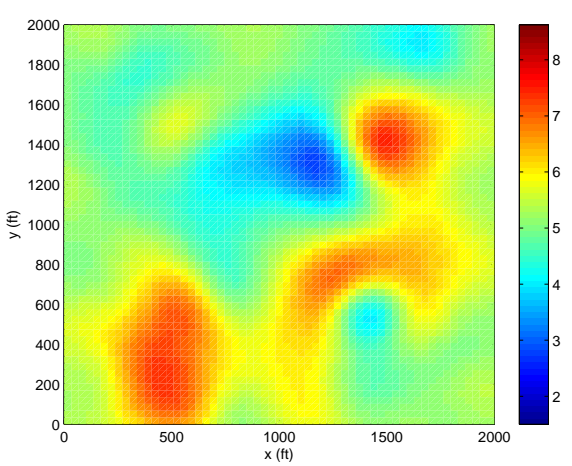

(C) Experiment IIB

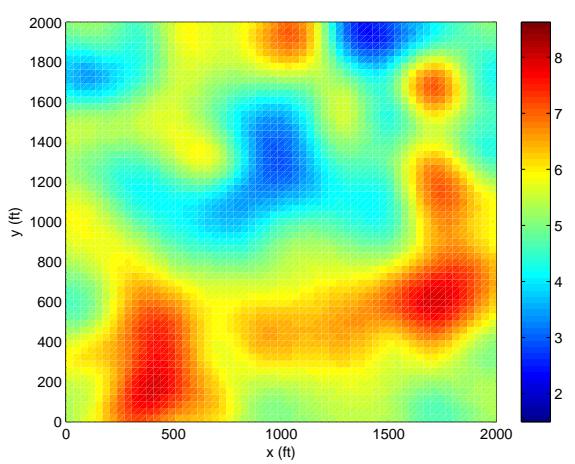

(E) Experiment IID

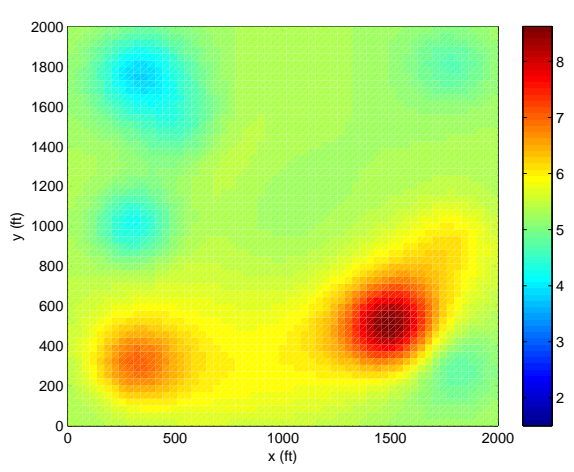

(B) Experiment IIA

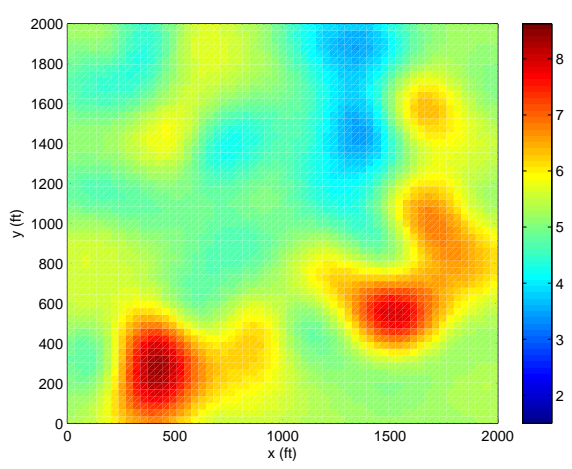

(D) Experiment IIC

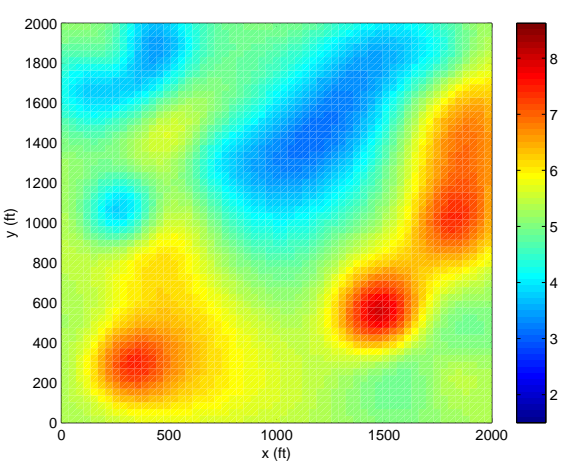

(F) Experiment IIE

FIgURE 4.2: Experiment II. Log permeability fields [n md] 
TABLE 4.5: Numerical Results. Experiment II.

\begin{tabular}{|c|c|c|c|c|c|}
\hline Configuration & IIA & IIB & IIC & IID & IIE \\
\hline \# Measu. Loc. & 8 & 16 & 25 & 36 & 8 \\
\hline \# of Meas. Times & 1 & 1 & 1 & 1 & 2 \\
\hline$\tau \eta[\mathrm{psi}]$ & 28.28 & 40.00 & 50.00 & 60.00 & 40.00 \\
\hline $\mathrm{a}, \mathrm{b}\left\|Y_{\text {true }}-Y^{*}\right\|_{l^{2}}[\ln (\mathrm{md})]$ & 68.74 & 63.75 & 58.08 & 47.27 & 59.84 \\
\hline Iterations & 5 & 3 & 4 & 4 & 6 \\
\hline
\end{tabular}

\section{Experiment III. Spe Model.}

In Experiment III we are interested in the estimation of a more realistic permeability field. The true permeability (Figure 6.13 (a)) is taken from the third layer of the absolute permeability data for the 10th SPE comparative project [33]. One of the interesting aspects of this permeability is that it has a variability of several orders of magnitude $\left(10^{-3} \mathrm{md}-10^{4} \mathrm{md}\right)$. A heterogeneous field of this type is challenging even for the direct simulation of reservoir models. Any inverse approach that attempts its reconstruction should be robust enough to manage, not only the number of degrees of freedom, but also its lack of regularity. We test the methodology with the forward model given by (3.1), for the estimation of the permeability from synthetic data (pressure). Measurements are collected at the injection and production wells, as wells as two additional observation wells. Table 4.3 show the reservoir description and Table 4.6 presents the information for the assimilation experiments. Figure 4.4 (a) and 4.4 (b) show the convergence history for both experiments. From Figure 6.13 (a) it is clear that the true log permeability is anisotropic. Although the isotropic covariance is only poor prior knowledge of the spatial variability, the estimated log permeability Figure 6.13 (b) capture some of the main characteristics of the true log permeability field. In Experiment IIIB we select, by inspection, a different error covariance function that reflects the higher spatial correlation in the $x$ direction. According to definition of noise level (Section 4.2.5), both experiments have the same stopping threshold. In fact, from Figure 4.4 (b) it can be observed that data has been fitted with about the same accuracy. However, in Experiment IIIB, an improved estimation is achieved (Figure 4.4 (a)) because we considered "better" prior knowledge (correlation lengths) than in Experiment IIIA. 
TABLE 4.6: Data Assimilation Information. Experiment IIIA and IIIB.

\begin{tabular}{ccc}
\hline Experiment & IIIA & IIIB \\
\# of Measurement Locations & 12 & 12 \\
\# of Measurement Times & 2 & 2 \\
$\tau$ & 2.0 & 2.0 \\
$\tau \eta[\mathrm{psi}]$ & 73.485 & 73.485 \\
$\left.\sigma_{\eta}[\mathrm{psi})\right]$ & 7.5 & 7.5 \\
$\bar{Y}[\ln \mathrm{md}]$ & 24.85 & 24.85 \\
${ }^{\mathrm{a}} C_{Y}\left(\xi, \xi^{\prime}\right)[\mathrm{ln} \mathrm{md}]^{2}$ & $3.2 \exp \left[-\frac{\| \xi-\xi^{\prime}||}{500 \mathrm{ft}}\right]$ & $3.2 \exp \left[-\sqrt{\frac{\left|x-x^{\prime}\right|^{2}}{(286 \mathrm{ft})^{2}}+\frac{\left|y-y^{\prime}\right|}{(160 \mathrm{ft})^{2}}}\right]^{\mathrm{a}}$ \\
\hline & $\mathrm{a} \xi=(x, y)$ &
\end{tabular}

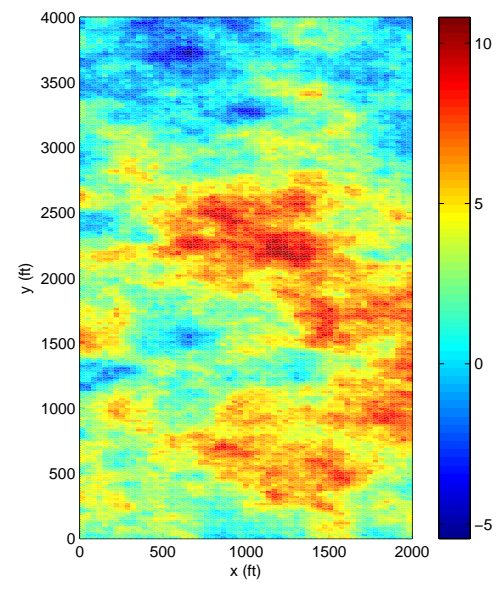

(A) True

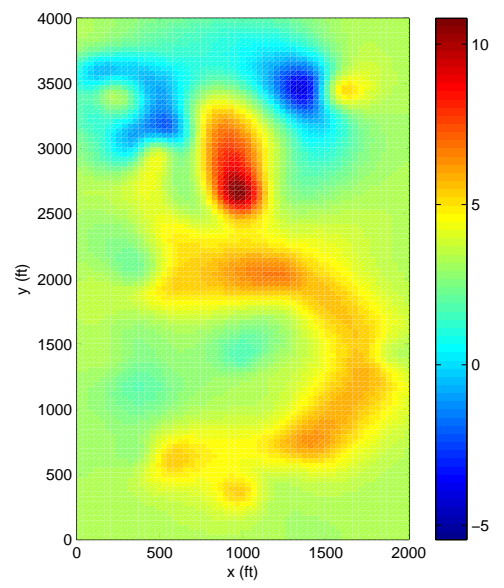

(B) Experiment IIIA

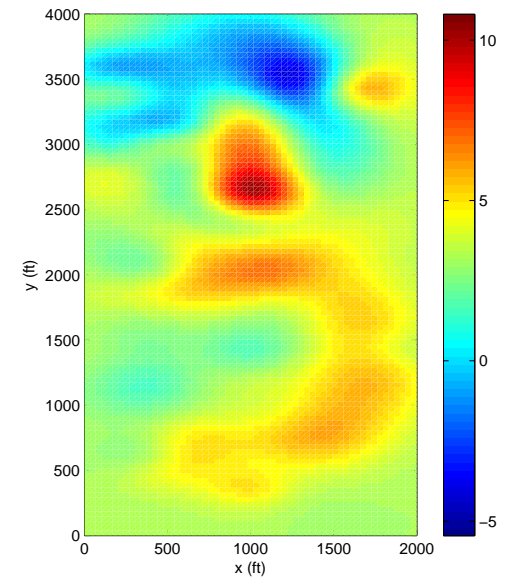

(C) Experiment IIIB

Figure 4.3: Experiment III. Log permeabilities [n md]. 


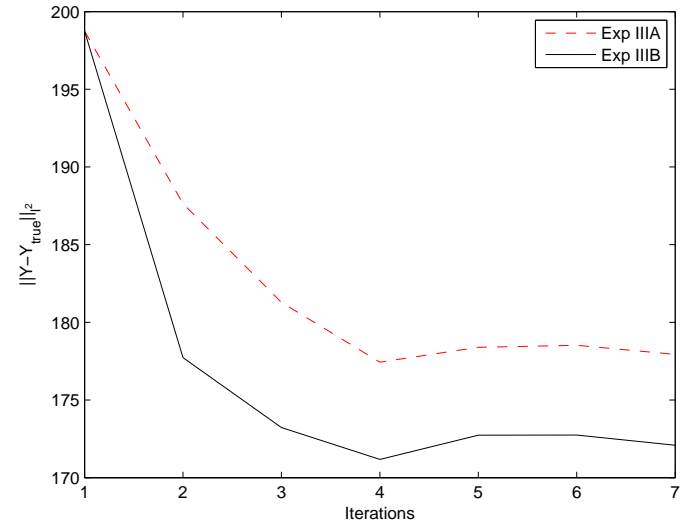

(A) $l^{2}$-Error in $Y=\ln K$

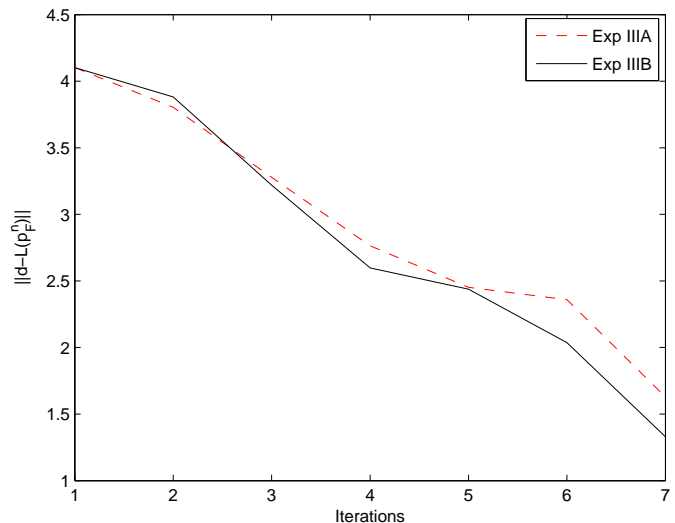

(B) log-measurement error $\log _{10}\left\|d-\mathcal{L}\left(p_{F}^{n}\right)\right\|$

FiguRE 4.4: Experiment III. Error performance. 


\section{Chapter 5}

\section{The IRBS with geological constraints}

In Chapter 4 we evaluated the efficiency of the IRBS for the estimation of absolute permeability in single-phase Darcy flow through porous media. The mean and the error covariance was the a priori information that we used in the implementation of the IRBS. Even when poor prior information was considered, the experiments of Chapter 4 show that the IRBS provides an acceptable reconstruction of permeability field. However, as we pointed in Chapter 1, any inversion technique must allow incorporation of any additional geological constraints. In this chapter we test the IRBS under geological constraints described by two-point statistics. Since the IRBS is a variational technique, we use Karhunen-Loeve (KL) expansions to parameterize the petrophisical properties of the reservoir. Finally, since the KL expansion has already been applied [60] for gradient-based history-matching techniques, we conduct a comparison of the IRBS-KL with a gradient-based approach whose optimization is performed with the Broyden-Fletcher-Goldfarb-Shanno (BFGS) method.

\subsection{Parametrization by K-L expansions}

In this section we discuss some of the key ideas in K-L expansions. For additional technical details we refer the reader to $[62,34]$. Let us consider a covariance operator $C$ defined on a Hilbert space $H$. Formally, when the covariance is a compact self-adjoint operator, the spectral theorem ensures the existence of an orthonormal basis $\left\{v_{\alpha}\right\}_{\alpha \in \mathcal{I}}$ of $H$, such that each $v_{\alpha}$ is an eigenvector of $C$. Additionally, for every $z \in H$ the following relation holds

$$
C(z)=\sum_{\alpha \in \mathcal{I}} \lambda_{\alpha}<v_{\alpha}, z>v_{\alpha}
$$


where $\lambda_{\alpha}$ is the eigenvalue of the corresponding eigenvector $v_{\alpha}$. In the context of Chapter 4 , the covariance is an integral operator on a functional space for which the previous expression becomes,

$$
C(x, y)=\sum_{i=1}^{\infty} \lambda_{i} v_{i}(x) v_{i}(y)
$$

and each $v_{i}$ is the solution of the following integral equation of the second kind

$$
\int_{\Omega} C(y, x) v_{i}(x) d x=\lambda_{i} v_{i}(y)
$$

With the previous considerations, it can be shown that a random field $Y$ with mean $\bar{Y}$ and covariance $C$ can be expressed as

$$
Y(x)=\bar{Y}(x)+\sum_{i}^{\infty} \sqrt{\lambda_{i}} \xi_{i} v_{i}(x),
$$

where $\left\{\xi_{i}\right\}_{i=1}^{\infty}$ is a family of uncorrelated random variables. Expression (5.4), usually referred to as the K-L expansion of $Y$, provides a parametrization of the random field $Y$ in terms of $\left\{\xi_{i}\right\}_{i=1}^{\infty}$, and deterministic variables $v_{i}$ and $\lambda_{i}$. From (5.2)-(5.4) is easy to see that the problem of finding the K-L expansions of a random field can be posed as a problem of finding the eigenvalues of the covariance operator.

In reservoir modeling applications, (5.4) can be used to characterized uncertain petrophysical properties with given two-point statistics. This may become more evident if we consider the discrete case for which (5.4) becomes

$$
w=\bar{w}+V D^{1 / 2} \xi
$$

where $V$ and $D$ are the matrices of eigenvectors and eigenvalues of the covariance matrix $C$. Therefore, once the eigenvalue decomposition is performed, a direct multiplication by an uncorrelated random vector will produce a realization $w$ with mean $\bar{w}$ and covariance $C$. Unfortunately, solving the eigenvalue problem may become computationally prohibitive since $C$ has dimensions $N \times N$ where $N$ is the number of grid blocks used to describe the reservoir. Additionally, in reservoir modeling the petrophysical information may be available from training images based on geological information such as the depositional environment of the reservoir. Therefore, the functional relation of the covariance may not be available. On the other hand, either from a covariance or a training image we can always obtain equally probable stochastic realizations of the petrophysical properties of the 
reservoir. Therefore, it has been suggested [59] to work in the general framework of using the covariance of a sample of stochastic realizations generated with the provided geological information. In the following lines we describe the standard procedure to find the K-L expansions when the covariance is computed from a sample.

Assume that we are given $N_{r}$ (zero mean) centered realizations $\left\{w_{j}\right\}_{j=1}^{N_{r}}$ of the petrophysical property $w$. Suppose that $N_{r}<N$ and define the covariance of the sample by

$$
C=\frac{1}{N_{r}} \sum_{j=1}^{N_{r}} w_{j} w_{j}^{T} .
$$

By construction $C$ cannot have more than $N_{r}-1$ nonzero eigenvalues. Let $N_{d}$ be the number of nonzero eigenvalues. Let us define $\tilde{D}$ the matrix of nonzero eigenvalues of $C$ sorted in ascending order and $\tilde{V}$ be the corresponding matrix of eigenvectors. From (5.4) is easy to see that zero eigenvalues are irrelevant for the parametrization of $w$ in terms of $\xi$. In fact, after a possible rearrangement of (5.4) the corresponding parametrization of $w$ is given by

$$
w=\bar{w}+\tilde{V} \tilde{D}^{1 / 2} \xi
$$

for $\xi \in \mathbb{R}^{N_{d}}$. Furthermore, any eigenvector of $C$ with nonzero eigenvalue must lie in the subspace generated by $\left\{w_{j}\right\}_{j=1}^{N_{r}}$. From this observation, it can be shown [62] that problem (5.3), for the discretized case and for nonzero eigenvalues, is equivalent to solve

$$
P u=N_{r} \lambda u
$$

where $P$ is the $N_{r} \times N_{r}$ kernel matrix defined by

$$
P_{i, j}=w_{i}^{T} w_{j}
$$

Since typically $N_{r}<<N$, there is an exceptional computational savings in solving (5.8) instead of the eigenvalue problem for $C$. Then, after solving (5.8) it is straightforward to find $\tilde{V}$ and $\tilde{D}$ respectively. Moreover, it is also possible to conduct additional reductions by keeping only $N_{K L}<N_{d}$ eigenvectors in $\tilde{V}$ corresponding to the highest eigenvalues. These eigenvectors, also called principal components, may retain enough information of the spatial variability of the petrophysical property consistent with the covariance $C$. This reduction, 
in the abstract setting can be written as a truncation of (5.4), i.e,

$$
Y(x)=\bar{Y}(x)+\sum_{i}^{N_{K L}} \sqrt{\lambda_{i}} \xi_{i} v_{i}(x) .
$$

Convergence results and other mathematical considerations with (5.10) can be found in [34].

\subsection{The IRBS with K-L parametrization}

Given prior geological information of the absolute permeability of a reservoir, in this section we apply the IRBS for the permeability estimation given production data from a single-phase simulator. The methodology follows the same steps as in the previous chapter. However, we incorporate the KL expansions so that the identification is performed for $\xi$ in (5.10). The IRBS with KL parametrization can be implemented with

Algorithm 2 (IRBS-KL). Assume we are given the prior mean of the petrophysical property $Y^{0}=\bar{Y}$ as well as the prior error covariance $C_{Y}$. Suppose $Y$ has a parametrization (5.4) in terms of $K-L$ expansions.

(1) Compute the spectral decomposition of $C_{Y}$.

(2) Determine $N_{K L}$ such that the first $N_{K L}$ eigenvalues retains enough information provided by $C_{Y}$ (i.e., that $Y$ can be described with (5.10)).

(3) Set $\xi^{0}=0$ (equivalently $Y^{0}=\bar{Y}$ ). For $n=1, \ldots$

[3.1] We compute $p_{F}^{n}$, the solution to the forward model

$$
G\left(\xi^{n-1}, p_{F}^{n}\right) \equiv\left[\begin{array}{c}
\frac{\partial p_{F}^{n}}{\partial t}-\nabla \cdot e^{Y\left(\xi^{n-1}\right)} \nabla p_{F}^{n}-f \\
-e^{Y\left(\xi^{n-1}\right)} \nabla p_{F}^{n} \cdot \mathbf{n}-B_{N} \\
p_{F}^{n}(x, 0)-I(x) \\
Y\left(\xi^{n-1}\right)-\bar{Y}-\sum_{i}^{N_{K L}} \sqrt{\lambda_{i}} \xi_{i}^{n-1} v_{i}(x)
\end{array}\right]=0
$$

[3.2] If $\left\|d^{\eta}-\mathcal{L}\left(p_{F}^{n}\right)\right\| \leq \tau \eta$, stop. Output: $Y\left(\xi^{n-1}\right)$.

[3.3] Solve

$$
\begin{array}{r}
\mathcal{Q}^{n}[\xi, p, \lambda]=[d-\mathcal{L}(p)]^{T} C_{\eta}^{-1}[d-\mathcal{L}(p)] \\
+\int_{\Omega} \int_{\Omega}\left[Y(\xi(x))-Y\left(\xi^{n-1}(x)\right)\right] C_{Y}(x, z)\left[Y(\xi(z))-Y\left(\xi^{n-1}(z)\right)\right] \\
+\int_{\Omega} \int_{0}^{T} \lambda L G(p, Y(\xi)) \rightarrow \text { min }
\end{array}
$$


where

$L G(p, \xi) \equiv\left[\begin{array}{c}c \frac{\partial p}{\partial t}-\nabla \cdot\left(e^{Y\left(\xi^{n-1}\right)} \nabla p\right)-\nabla \cdot\left[D_{\xi} Y\left(\xi^{n-1}\right)\left[\xi-\xi^{n-1}\right] e^{Y\left(\xi^{n-1}\right)} \nabla p_{F}^{n}\right]-f \\ \left.\left(e^{Y\left(\xi^{n-1}\right)} \nabla p \cdot \mathbf{n}+D_{\xi} Y\left(\xi^{n-1}\right)\left[\xi-\xi^{n-1}\right] e^{Y\left(\xi^{n-1}\right)} \nabla p_{F}^{n} \cdot \mathbf{n}\right)\right|_{\Gamma_{N}}-B_{N} \\ \left.p\right|_{t=0}-I\end{array}\right]=0$

and

$$
D_{\xi} Y\left(\xi^{n-1}\right)\left[\xi-\xi^{n-1}\right]=\sum_{i}^{N_{K L}} \sqrt{\lambda_{i}} v_{i}(x)\left[\xi-\xi^{n-1}\right] .
$$

\subsubsection{Some Remarks on the IRBS-KL}

We now discuss some interesting and useful aspects of Algorithm 2. First, we note that constraint (5.14) can be incorporated directly in (5.12) without the need of an additional adjoint. Second, the representer method can be used to solve the E-L equations resulting from (5.12). It follows trivially that (4.42) must be replaced with

$$
\gamma_{m}^{n}(x)=-\sum_{i=1}^{N_{k l}} \int_{0}^{T} \int_{\Omega} \lambda_{i} v_{i}(x) v_{i}(y) \nabla \alpha_{m}^{n}\left(x^{\prime}, t\right) \cdot e^{Y\left(\xi^{n-1}\left(x^{\prime}\right)\right)} \nabla p_{F}^{n}\left(x^{\prime}, t\right) d t d x^{\prime} .
$$

where now $\gamma_{m}^{n}$ corresponds to the representer expansion of $\xi$, i.e.

$$
\xi^{n}=\xi^{n-1}+\sum_{m=1}^{M} \beta_{m}^{n} \gamma_{m}^{n}
$$

Furthermore, we observe that the minimization of (5.12) is conducted for $\xi$ instead of $Y$. Then, in order to understand the regularizing effect of the IRBS, we study the second term in the right hand side of (5.12). From (5.11) note that

$$
\begin{array}{r}
\int_{\Omega} \int_{\Omega}\left[Y(\xi(x))-Y\left(\xi^{n-1}(x)\right)\right] C_{Y}(x, z)\left[Y(\xi(z))-Y\left(\xi^{n-1}(z)\right)\right] \\
=\sum_{i, j}^{N_{K L}} \int_{\Omega} \int_{\Omega} \sqrt{\lambda_{j}} \sqrt{\lambda_{i}}\left[\xi_{i}-\xi_{i}^{n-1}\right]\left[\xi_{i}(z)-\xi_{i}^{n-1}(z)\right] v_{i}(x) v_{j}(z) \\
=\sum_{i, j}^{N_{K L}} \int_{\Omega} \int_{\Omega} \lambda_{i}\left[\xi_{i}-\xi_{i}^{n-1}\right]^{2}=\left\|\xi-\xi^{n-1}\right\|_{L^{2}(\Omega)}
\end{array}
$$


where we have used the fact that $\left\{v_{i}\right\}_{i=1}^{N_{K L}}$ is orthonormal. Then, (5.17) states that the identification on $\xi$ penalizes only the $L^{2}$-norm of $\xi$ and therefore no regularity is insured. Then, any regularity on $Y$ is now obtained by the KL parametrization. This statement should have some implications in the identification of parameters with IRBS-KL as we will point out in the following section. Finally, we recall from the previous chapter that the IRBS is data driven in the sense that convergence is controlled by the misfit between the observations and model predictions computed at each iteration $\left(\| d^{\eta}-\mathcal{L}\left(p_{F}^{n}\right)\right)$. More precisely (see Theorem (3)), convergence is achieved when $\| d^{\eta}-\mathcal{L}\left(p_{F}^{n}\right) \leq \tau \eta$. Therefore, changing the parametrization of the identified variables does not affect the implementation of the stopping rule. However, from Theorem 3 and (2) it is clear that the parametrization will affect the radius and speed of convergence of the IRBS.

\subsubsection{A Numerical Example}

Let us consider the anisotropic "true permeability" of Figure 5.3 (A). This stochastic field was generated with SGSIM, assuming an exponential variogram with a maximum range of continuity in the north-east direction. The corresponding covariance can be obtained by the following relation

$$
C_{Y}(h)=C \exp \frac{-h^{\prime}}{a}
$$

where $h=(x, y), a$ is the range in the north-east direction and $h^{\prime}=A h$ with

$$
A=\left[\begin{array}{cc}
\cos (\pi / 4) & \sin (\pi / 4) \\
r \sin (\pi / 4) & r \cos (\pi / 4)
\end{array}\right] .
$$

In (5.17), $r=a / a_{\text {min }}$ is the anisotropic ratio which for this experiment we chose $r=10$. In Experiment IV-A, we apply the IRBS as in the previous chapter with the covariance given by (5.18) and without any parametrization. The reservoir description is displayed in Table 5.1 and convergence is shown in Table 5.2. The corresponding reconstruction is presented in Figure 5.3 (b). Note that the IRBS has produced a remarkable estimation since the major heterogeneities have been recovered. However, the estimated field is smoother than the "true permeability" that we attempted to reproduce. This is not surprising when we observe that (5.18) is used in the right hand side of (4.34). Formally, $Y(x)$ will inherit the regularity of $C_{Y}(x, z)$, which from (5.18) is $C^{1}$ in $\left\{x \in \mathbb{R}^{2}-\{z=x\}\right\}$. Therefore, we expect $Y$ to be at least continuous as we indeed confirm by looking at Figure 5.3(b).

We now apply the IRBS with the K-L parametrization presented in the previous 
TABLE 5.1: Reservoir description. Experiments $I V(A)-I V(C)$

\begin{tabular}{lc|lc}
\hline Dimension $\mathrm{ft}^{3}$ & $800 \times 800 \times 10$ & Water viscosity $(\mathrm{cp})$ & 0.3 \\
Grid Blocks (for IRBS) & $80 \times 80 \times 1$ & Initial pressure (psi) & 5000 \\
Time interval $[0, T]$ (days) & {$[0,1]$} & Number Injection Wells & 6 \\
Time discretization $d t$ (days) & 0.01 & Number Production Wells & 2 \\
Porosity & 0.2 & Shut-in Time $t_{s}($ day) & 0.75 \\
Water compressibility $\left(\mathrm{psi}^{-1}\right)$ & $3.1 \mathrm{e}-6$ & ${ }^{a}$ Injection rate $(\mathrm{stb} /$ day) & 833.33 \\
Rock compressibility $\left(\mathrm{psi}^{-1}\right)$ & $1.0 \mathrm{e}-6$ & ${ }^{a}$ Production rate (stb/day) & 1200 \\
\hline
\end{tabular}

${ }^{\text {a }}$ Rate per well in the interval $\left[0, t_{s}\right]$.

section. With SGSIM we generate 180 unconditional stochastic realizations consistent with (5.18). Some of those realizations are presented in Figure (5.1). From a geological point of view, those fields are equally probable permeabilities consistent with the geology, which in this case, is given by covariance (5.18) and a prior mean $\bar{Y}=\log (450 \mathrm{md})$. It is now the goal of the IRBS-KL to find among all those possible fields, one that produces the best fit to the production data in the sense of Definition ??.

We start Algorithm 2 by computing the kernel eigenvalue decomposition (5.8) of the sample covariance defined in (5.6), also denoted by $C_{Y}$. In Figure 5.2(a) we present a stochastic field generated with parametrization (5.7). Note that this field has the same structure as those of Figure 5.1 and so it is indeed a good parametrization of the underlying heterogeneities. Figures 5.2(b)-(d) show the same field but generated with only some of the highest eigenvectors of $C_{Y}$. It can be observed that $50 \%\left(N_{K L}=90\right)$ of the highest eigenvalues is enough to retain the spatial structure. Therefore, in Experiment IV(B) we use parametrization (5.7) for the first 90 eigenvalues of $C_{Y}$ and we repeat Experiment IV(A) but now using IRBS as we indicated in Algorithm 2. In Figure 5.3 (c) we present the estimated permeability field. Note that this estimation satisfies the geological constraints imposed by the prior error covariance. In Table 5.2 the convergence history for Experiment $\mathrm{IV}(\mathrm{B})$ is displayed. Since the number of measurements is the same, the stopping threshold is also the same for both experiments $\operatorname{IV}(\mathrm{A})$ and $\operatorname{IV}(\mathrm{B})$ (see Section 4.2.5). However, as we pointed out earlier, convergence is slower in the IRBS-KL because the parametrization affects the rate of convergence. However, as we expected the IRBS-KL produces a bigger reduction of the error in the permeability field. 


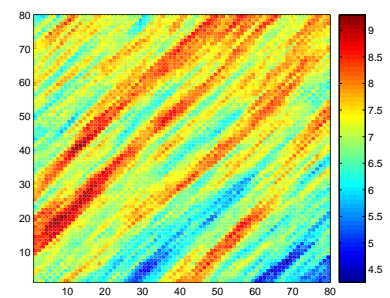

(A) Realization 1

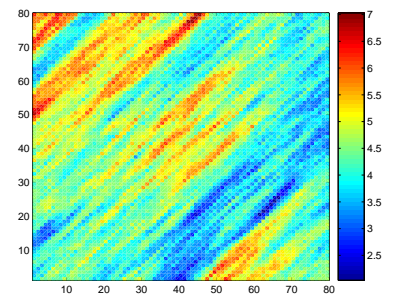

(D) Realization 4

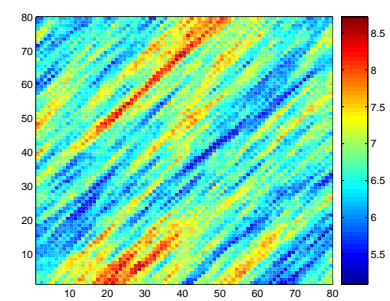

(B) Realization 2

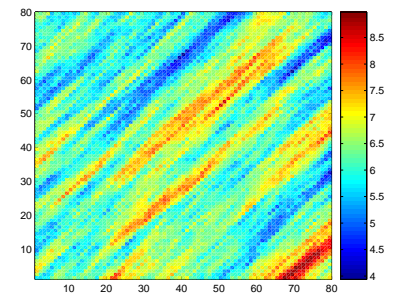

(E) Realization 5

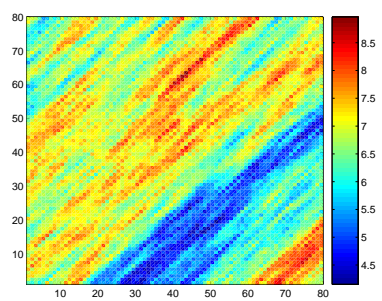

(C) Realization 3

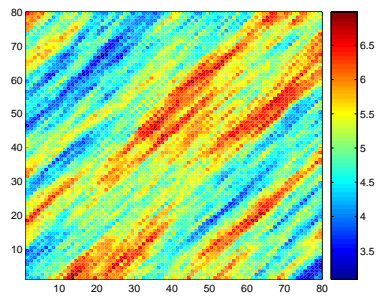

(F) Realization 6

FIgURE 5.1: Experiment IV. Some stochastic realizations generated for the application of the IRBS-KL

TABLE 5.2: Numerical Results. Experiment IV.

\begin{tabular}{lllll}
\hline & Experiment IVa & & Experiment IVb \\
$n$ & $\left\|Y^{n-1}-Y_{\text {true }}\right\|_{l^{2}}$ & $\left\|d^{\eta}-\mathcal{L}\left(p_{F}^{n}\right)\right\|$ & $\left\|Y^{n-1}-Y_{\text {true }}\right\|_{l^{2}}$ & $\left\|d^{\eta}-\mathcal{L}\left(p_{F}^{n}\right)\right\|$ \\
1 & 67.7438 & $0.3280 \mathrm{e} 3$ & 67.7438 & $1.2176 \mathrm{e} 3$ \\
2 & 67.8908 & $1.8866 \mathrm{e} 3$ & 91.5568 & $8.7531 \mathrm{e} 3$ \\
3 & 85.0330 & $0.5446 \mathrm{e} 3$ & 59.6032 & $2.9214 \mathrm{e} 3$ \\
4 & 53.5765 & $0.1449 \mathrm{e} 3$ & 43.2175 & $0.8300 \mathrm{e} 3$ \\
5 & 48.8545 & $0.0246 \mathrm{e} 3$ & 38.4377 & $0.1539 \mathrm{e} 3$ \\
6 & - & - & 37.9704 & $0.0138 \mathrm{e} 3$ \\
\hline
\end{tabular}




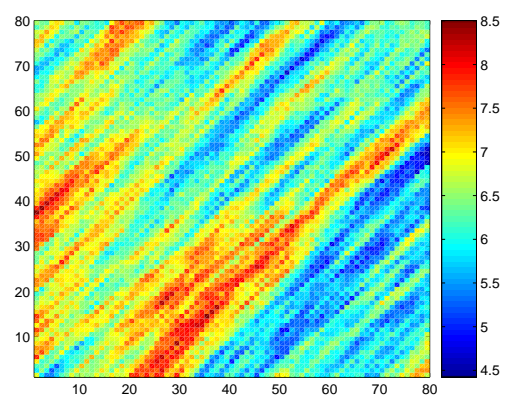

(A) $100 \%$

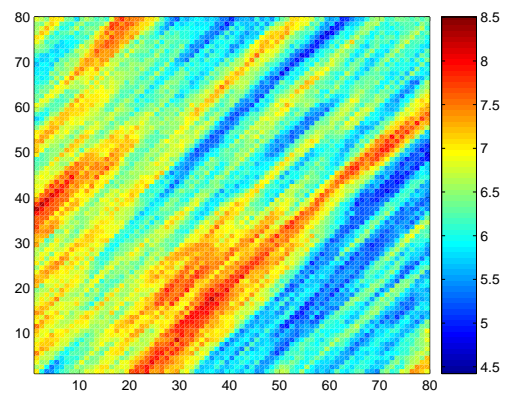

(C) $50 \%$

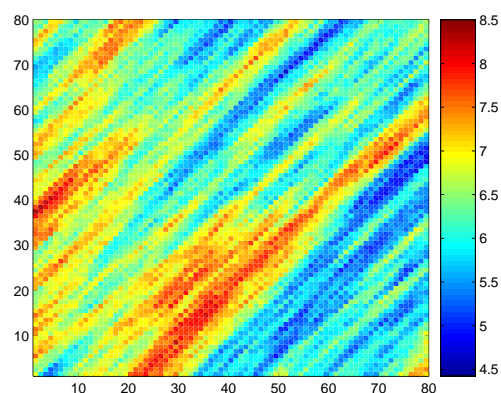

(в) $70 \%$

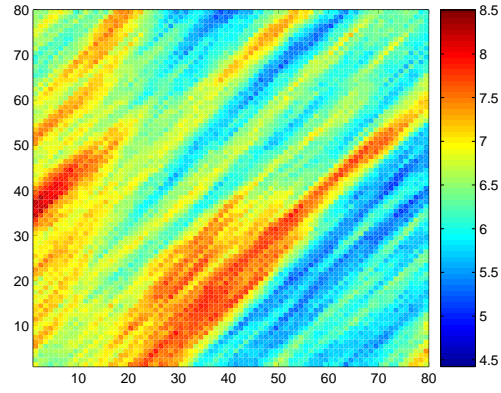

(D) $30 \%$

FiGURE 5.2: Example of a field generated with $K L$ expansions, retaining some percentage of the highest eigenvalues values 


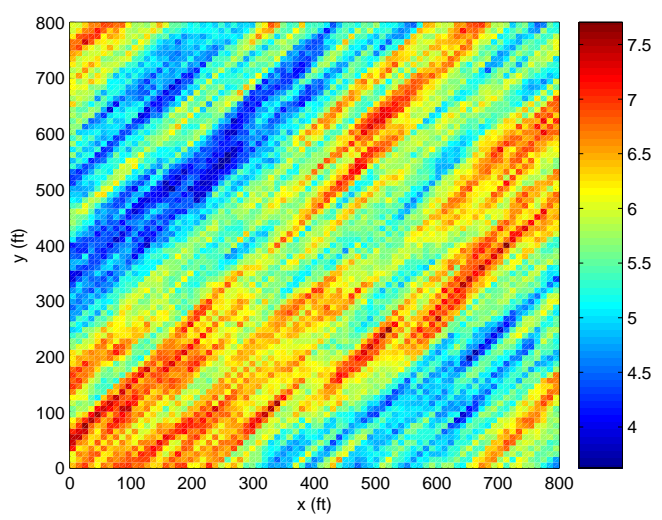

(A) True

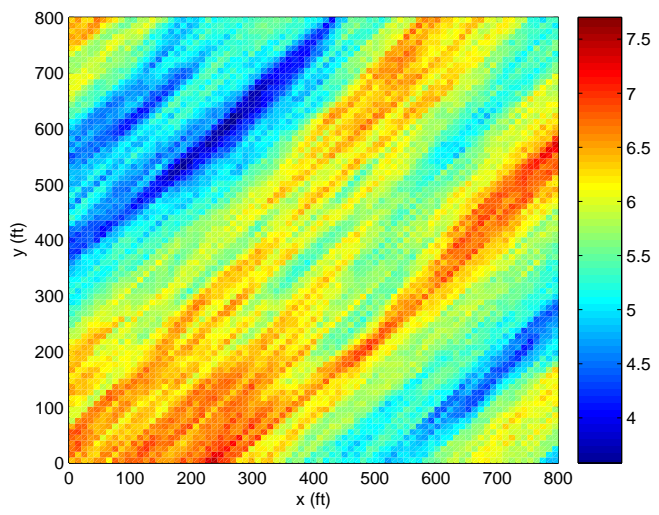

(C) IRBS with $K L$

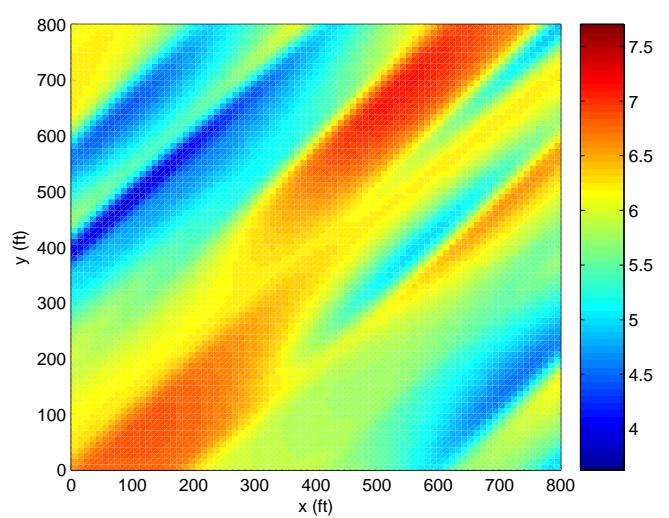

(B) $\operatorname{IRBS}$

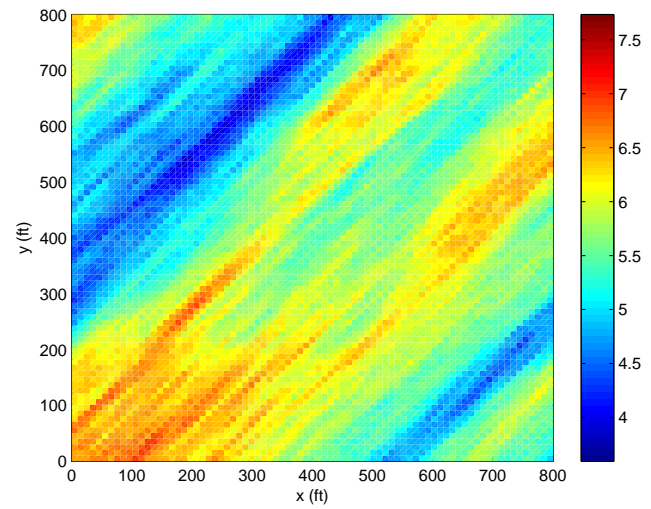

(D) Gradient Based (BFGS)

Figure 5.3: Experiment IV. Permeability Fields. 


\subsection{The IRBS vs. a standard Gradient-Based Technique}

As we study in the previous section, the parametrization by KL expansions (5.4) not only provides computational savings but also ensure geological consistency (two-point statistics) of the estimated field. Additionally, we showed that the KL expansions can be incorporated to the IRBS because (5.4) is a differentiable operator (see equation (5.14)). Therefore, the KL parametrization can be applied to any gradient-based history matching approach. In this section we study the implementation of the standard variational approach for history matching with geological constraints. We use the single phase simulator of Chapter 4 and the same reservoir of Experiment $\operatorname{IV}(\mathrm{A})$.

\subsubsection{The standard Variational Approach}

We now address the "parameter estimation" problem with the standard variational approach [28]. For the model of Chapter 4, this formulation is posed by the minimization of

$$
J=[d-\mathcal{L}(p)]^{T} C^{-1}[d-\mathcal{L}(p)]+\int_{\Omega} \int_{\Omega}[Y-\bar{Y}] C_{Y}[Y-\bar{Y}] \rightarrow \min
$$

subject to

$$
G(p, Y) \equiv\left[\begin{array}{c}
c \frac{\partial p}{\partial t}-\nabla \cdot\left(e^{Y} \nabla p\right)-f \\
-e^{Y} \nabla p \cdot \mathbf{n}+B_{N} \\
\left.p\right|_{t=0}-I
\end{array}\right]=0
$$

where $d$ is the vector of pressure measurements with covariance matrix $C, \mathcal{L}$ is the corresponding linear functional applied to the $p$ computed from (5.21). When $Y$ and $d$ follows a Gaussian distribution with prior error covariance $C_{Y}$ and $C$ respectively, then (5.20) can be derived from a Bayesian formulation [28]. In that case, (5.20) is equivalent to maximizing the conditional joint probability density function $f(p, Y \mid d)$. If the forward model (5.21) were linear (in $(p, Y)$ ), then "the" solution to (5.20) would be the (most probable) maximum a posteriori estimate of $Y$. However, since the constraint $G$ is nonlinear, the solution to (5.20) may not be unique and therefore the concept of "most probable" estimate does not apply. Nevertheless, the estimation problem, with the standard variational approach, for the nonlinear case, is to find a local minimum of $J$. 


\subsubsection{A gradient-based algorithm}

Among the several techniques that can be used to minimize $J$, the BFGS method has received special attention since it does not involves the computation of the Hessian of $J$. Although we are considering a toy problem for which any other technique may be suitable, we use the BFGS to find a solution of (5.20). The BFGS method involves the computation of the derivative of $J$ with respect $Y$ which required the adjoint of $G$. It is not difficult to see that

$$
\frac{\partial J}{\partial \xi}(p, \lambda, \xi)[\delta \xi]=\int_{\Omega} \int_{\Omega}[Y(x)-\bar{Y}(x)] C_{Y}(x, z) D_{\xi} Y \delta \xi(z)+\int_{0}^{T} \int_{\Omega} \nabla \lambda \cdot e^{Y} \nabla p D_{\xi} Y \delta \xi
$$

where $\lambda$ is the solution to

$$
\begin{aligned}
& -\frac{\partial \lambda}{\partial t}-\nabla \cdot\left(e^{Y} \nabla \lambda\right)=(d-\mathcal{L}(p)) C^{-1} H \quad \text { in } \Omega \times(0, T], \\
& e^{Y} \nabla \lambda \cdot \mathbf{n}=\quad 0 \quad \text { on } \Gamma_{N} \times(0, T], \\
& \lambda=\quad 0 \quad \text { in } \Omega \times\{T\} .
\end{aligned}
$$

Here $H=\left[H_{1} \ldots, H_{m}\right]$ and each $H_{m}$ is defined by (4.3). In (5.22) we allow for some differentiable parametrization $Y=Y(\xi)$ for $\xi \in L^{2}(\Omega)$. The standard variational approach with the BFGS is summarized in the following

Algorithm 3 (Standard Variational Approach with BFGS). Given a priori mean $\bar{\xi}=0, \bar{Y})$, prior error covariances $C_{Y}$ and $C$. For $n=1, \ldots$

(1) Start with $H_{n-1}=H_{0}$ an approximation to the Hessian of $J$

(2) Determine a direction of descent $\delta \xi$ by solving

$$
\left\langle H^{n-1} \delta \xi, \tilde{\delta \xi}\right\rangle_{L^{2}(\Omega)}=-\frac{\partial J}{\partial \xi}\left(p^{n-1}, \lambda^{n-1}, \xi^{n-1}\right)[\tilde{\delta \xi}]
$$

for all $\tilde{\delta \xi} \in L^{2}(\Omega)$.

(3) Perform a line search to find $\xi^{n}=\xi^{n-1}+\alpha \delta \xi$ where $\alpha$ is chosen so that the strong Wolfe conditions are satisfied.

(4) Compute $p^{n}, \lambda^{n}$ and $\frac{\partial J}{\partial \xi}\left(p^{n}, \lambda^{n}, \xi^{n}\right)$ from (5.21), (5.23) and (5.22) respectively. If $\left\|\frac{\partial J}{\partial \xi}\right\|_{L^{2}(\Omega)} \leq \theta$, stop. Output: $\xi^{n}$. 
(5) Define the new Hessian approximation by

$$
\begin{aligned}
H_{n} v & =H_{n-1} v+A \frac{u_{n}}{\left\|u_{n}\right\|_{L^{2}(\Omega)}}\left\langle\frac{u_{n}}{\left\|u_{n}\right\|_{L^{2}(\Omega)}}, v\right\rangle_{L^{2}(\Omega)} \\
& +B \frac{H_{n-1} s_{n}}{\left\|H_{n-1} s_{n}\right\|_{L^{2}(\Omega)}}\left\langle\frac{H_{n-1} s_{n}}{\left\|H_{n-1} s_{n}\right\|_{L^{2}(\Omega)}}, v\right\rangle_{L^{2}(\Omega)}
\end{aligned}
$$

where

$$
u_{n}=\frac{\partial J}{\partial \xi}\left(p^{n-1}, \lambda^{n-1}, \xi^{n-1}\right)-\frac{\partial J}{\partial \xi}\left(p^{n}, \lambda^{n}, \xi^{n}\right) \quad s_{n}=Y^{n}-Y^{n-1}
$$

and

$$
A=\frac{\left\|u_{n}\right\|_{L^{2}(\Omega)}^{2}}{\left\langle u^{n}, s^{n}\right\rangle_{L^{2}(\Omega)}} \quad B=\frac{\left\|H_{n-1} s^{n}\right\|_{L^{2}(\Omega)}^{2}}{\left\langle u^{n}, H_{n-1} s^{n}\right\rangle_{L^{2}(\Omega)}}
$$

\subsubsection{Remarks on Algorithm 3}

We now remark some points with respect to Algorithm 3

(1) Algorithm (3) is an iterative scheme that finds a local minimum of $J$ constrained to the nonlinear equation (5.21). In contrast, on each iteration of the IRBS we solve a cost functional $J^{n}$ constrained to the linearization of (5.21).

(2) After convergence the BFGS finds $[p, \lambda, \xi]$ that solves the nonlinear E-L equations associated with (5.20). In other words, BFGS finds a solution to

$$
\frac{\partial J}{\partial \xi}=0
$$

On the other hand, the IRBS finds a solution to the PIP in the sense of Definition (??) which does not explicitly involve the minimization of a functional.

(3) The BFGS is stopped when $\frac{\partial J}{\partial \xi}$ is sufficiently small. The IRBS is terminated when the data misfit is small.

\subsubsection{Numerical Comparison}

To further observe the difference of the IRBS and the standard approach with BFGS we conduct Experiment IV(c) with the same reservoir whose (true) permeability is shown in Figure 5.3(a). The estimation has been conducted with Algorithm 3. At the discretized level, instead of using the approximation to the Hessian operator we use the following 


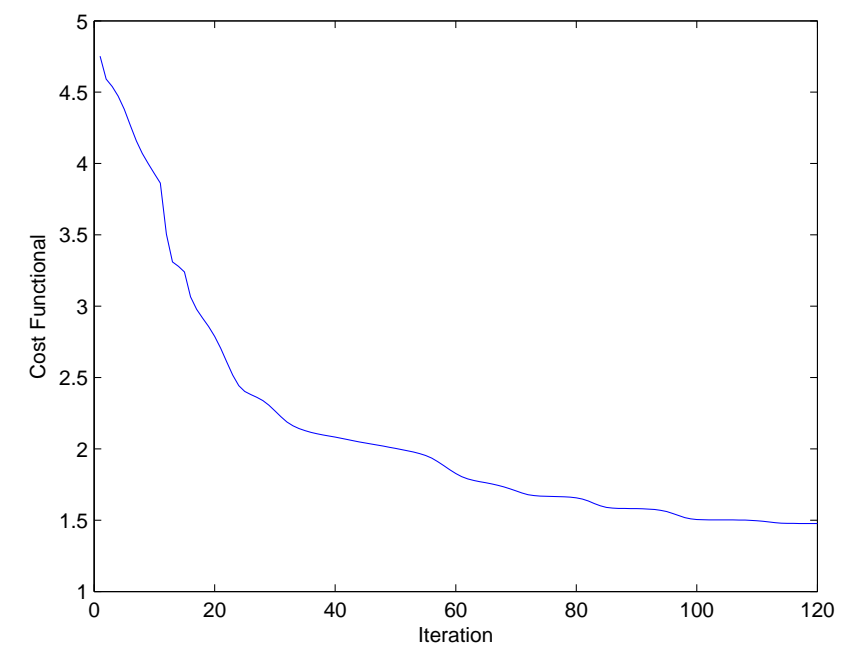

(A) True

Figure 5.4: Experiment IV(c). Performance of cost functional

expression

$$
H_{n}^{-1}=\left[I-\frac{u_{n} s_{n}^{T}}{u_{n}^{T} s_{n}}\right] H_{n-1}^{-1}\left[I-\frac{u_{n} s_{n}^{T}}{u_{n}^{T} s_{n}}\right]+\frac{s_{n} s_{n}^{T}}{u_{n}^{T} s_{n}}
$$

where $u_{n}$ and $s_{n}$ are the discretized versions of (5.28). For the line search of step (3), we implemented a back tracking algorithm that uses quadratic interpolation for the fulfillment of the strong Wolfe conditions. We selected an stopping threshold $\theta=1 e-3$ and an initial approximation to the inverse of the Hessian $H_{o}^{-1}=1 e-3 \mathbf{I}$. We will come back to this point in the next paragraph. The BFGS takes 120 iterations to converge (see Figure 5.4) and it requires 1200 forward model evaluations. In Figure 5.3(d) we present the estimated permeability field. After convergence the BFGS produces a field with an error of $\left\|Y_{\text {true }}-Y_{B F G S}\right\|_{l^{2}}=39.02$. A direct comparison shows that the IRBS generates a field (Experiment IV(b)) with slightly better accuracy $\left(\left\|Y_{\text {true }}-Y_{B F G S}\right\|_{l^{2}}=37.83\right.$. However, the IRBS required 6 iterations which accounts for 300 forward model evaluations. While the accuracy is equivalent, the IRBS performance is four times faster than the standard approach with BFGS. 


\section{Chapter 6}

\section{The IRBS for data inversion in two-phase flow through porous media}

In this chapter we describe the application of the IRBS for the identification of petrophysical properties in an oil-water reservoir. In Section 6.1 we introduce the forward model of interest and in Section 6.2 we define the measurement process for the inverse problem. The application of the IRBS is presented in Section 6.3. Some technical aspects of the implementation are provided in Section 6.4. In Section 6.5 we present two experiments for the estimation of petrophysical properties in this application. Finally, in Section 6.6 we propose an ad-hoc sequential implementation suitable for a closed-loop approach. Numerical results for a benchmark are obtained, and a comparison with the EnKF is provided.

\subsection{The Forward Model}

We consider the immiscible displacement of two-phase slightly compressible flow through a porous medium. Let us denote by $\alpha=w(\alpha=o)$ the corresponding wetting phase (nonwetting phase). We suppose that there are $N_{I}$ injection wells and $N_{P}$ production wells whose locations within the reservoir are $\left\{x_{I}^{l}\right\}_{l=1}^{N_{I}}$ and $\left\{x_{P}^{l}\right\}_{l=1}^{N_{P}}$ respectively. Moreover, it is assumed that only the wetting phase is injected at a specified rate denoted by $Q_{w}$. In addition, the production rate for each phase is denoted by $q_{\alpha}$ and we impose the condition that the corresponding production wells are operated under specified bottom hole pressure. The porosity and the absolute permeability of the reservoir are denoted by $\phi$ and $K$ where the latter is considered isotropic (with respect to the flow direction), and the former is 
assumed to satisfy the following relation

$$
\phi=\phi_{r}\left[1+c_{r}\left(p-p_{r}\right)\right]
$$

where $\phi_{r}$ is the porosity at pressure $p_{r}$ and $c_{r}$ is the (constant) rock compressibility. Let $s_{\alpha}$ and $\rho_{\alpha}$ be the saturation and the density of the $\alpha$-phase respectively. In this analysis we assume that $\rho_{\alpha}$ is given by

$$
\rho_{\alpha}=\rho_{\alpha}^{r}\left[1+c_{\alpha}\left(p-p_{r}\right)\right]
$$

where $\rho_{\alpha}^{r}$ is the density at pressure $p_{r}$ and $c_{\alpha}$ is the (constant) compressibility of the $\alpha$-phase. With these assumptions, the model equations can be written as

$$
\frac{\partial}{\partial t}\left(\phi \rho_{\alpha} s_{\alpha}\right)+\nabla \cdot\left(\rho_{\alpha} \mathbf{u}_{\alpha}\right)=\rho_{\alpha} \sum_{l=1}^{N_{P}} q_{\alpha}^{l} \delta\left(x-x_{P}^{l}\right)+\rho_{\alpha} \sum_{l=1}^{N_{I}} Q_{\alpha}^{l} \delta\left(x-x_{I}^{l}\right)
$$

where the flux for each phase is given by Darcy's law

$$
\mathbf{u}_{\alpha}=-\frac{k_{r \alpha}}{\mu_{\alpha}} K \nabla p
$$

In (6.4), $k_{r \alpha}$ denotes the relative permeability, $\mu_{\alpha}$ the viscosity and $p$ is the pressure. In equation (6.4), capillarity is neglected and so $p$ is the field pressure for both phases. The mobility for each phase and the total mobility are defined by

$$
\lambda_{\alpha} \equiv \frac{k_{r \alpha}}{\mu_{\alpha}}
$$

and

$$
\lambda \equiv \lambda_{w}+\lambda_{o}
$$

respectively. For the injection/production rates we use Peaceman's well model [54]

$$
\begin{aligned}
q_{\alpha}^{l}(t) & \equiv \omega_{P}^{l} \lambda_{\alpha} K\left[P_{b h}^{l}-p\right], \\
Q_{w}^{l}(t) & \equiv \omega_{I}^{l} \lambda K\left[p_{b h}^{l}-p\right],
\end{aligned}
$$

where the well index is defined by

$$
w_{\xi}^{l}=\frac{2 \pi \Delta L^{\xi, l}}{\ln \left(r_{e}^{(\xi, l)} / r_{w}^{(\xi, l)}\right)}
$$


for $\xi=\{I, P\}$. In expression (6.1), $\Delta L^{\xi, l}$ denotes the length of a gridblock containing the $l$ th injection $(\xi=I) /$ production $(\xi=P)$ well. $r_{e}^{\xi, l}$ and $r_{w}^{\xi, l}$ denote the equivalent and the well radius respectively. In definitions (6.7) and (6.8), $K, \lambda, \lambda_{\alpha}$ and $p$ are evaluated at the gridblock containing the corresponding well. However, for the sake of clarity in the subsequent analysis the notation of this evaluation will be omitted.

We now assume both phases fill the porous medium and so

$$
s_{w}+s_{o}=1 \text {. }
$$

Expanding the first two terms in (6.3), using (6.1)-(6.2), dividing by $\rho_{\alpha}$ and considering that $c_{r} \phi_{r} \approx c_{r} \phi$ and $c_{r}^{2} \approx 0$, we obtain

$$
\phi\left(c_{r}+c_{\alpha}\right) s_{\alpha} \frac{\partial p}{\partial t}+\phi \frac{\partial s_{\alpha}}{\partial t}+\nabla \cdot \mathbf{u}_{\alpha}=\sum_{l=1}^{N_{p}} q_{\alpha}^{l} \delta\left(x-x_{P}^{l}\right)+\sum_{l=1}^{N_{I}} Q_{\alpha}^{l} \delta\left(x-x_{I}^{l}\right)
$$

Adding (6.11) for both phases and using (6.10) we find

$$
\phi c\left(s_{w}\right) \frac{\partial p}{\partial t}+\nabla \cdot \mathbf{u}=\sum_{l=1}^{N_{p}} q^{l} \delta\left(x-x_{P}^{l}\right)+\sum_{l=1}^{N_{I}} Q_{w}^{l} \delta\left(x-x_{I}^{l}\right),
$$

where we have defined

$$
\begin{gathered}
c\left(s_{w}\right) \equiv\left(c_{r}+c_{w} s_{w}+c_{o}\left(1-s_{w}\right)\right), \\
q^{l} \equiv q_{w}^{l}+q_{o}^{l}, \\
\mathbf{u} \equiv \mathbf{u}_{w}+\mathbf{u}_{o} .
\end{gathered}
$$

Note from (6.4)-(6.5) and (6.15) that

$$
\begin{aligned}
\mathbf{u}_{w} & =f_{w} \mathbf{u}, \\
\mathbf{u} & =-\lambda K \nabla p,
\end{aligned}
$$

where the fractional flow is given by

$$
f_{w} \equiv \frac{\lambda_{w}}{\lambda} .
$$


Similarly we find that

$$
q^{l}=q_{w}^{l}+q_{o}^{l}=\omega_{P}^{l} \lambda K\left[P_{b h}^{l}-p\right]
$$

and so

$$
q_{w}=\frac{\lambda_{w}}{\lambda} q=f_{w} q
$$

With (6.16) and (6.20) we rewrite equation (6.11), for $\alpha=w$, as

$$
\phi\left(c_{r}+c_{w}\right) s_{w} \frac{\partial p}{\partial t}+\phi \frac{\partial s_{w}}{\partial t}+\nabla \cdot f_{w} \mathbf{u}=\sum_{l=1}^{N_{p}} f_{w} q^{l} \delta\left(x-x_{P}^{l}\right)+\sum_{l=1}^{N_{I}} Q_{w}^{l} \delta\left(x-x_{I}^{l}\right) .
$$

Additionally, for simplicity, Neumann boundary conditions are provided

$$
\mathbf{u} \cdot \mathbf{n}=B
$$

as well as initial conditions for pressure and water saturation

$$
p(0, x)=p_{0}, \quad s_{w}(0, x)=s_{0} .
$$

Finally, for each phase $\alpha$, we assume that the relative permeability $k_{r, \alpha}$ is a function of the phase saturation parameterized by

$$
\begin{gathered}
k_{r w}=a_{w}\left[\frac{s_{w}-s_{i w}}{1-s_{i w}-s_{o r}}\right]^{\theta_{w}}, \\
k_{r o}=a_{o}\left[\frac{1-s_{w}-s_{o r}}{1-s_{i w}-s_{o r}}\right]^{\theta_{o}} .
\end{gathered}
$$

Since only $s_{w}$ will be considered for the model equations, from now on the subscript will be omitted. Then, from (6.12), (6.21), (6.7)-(6.8) and (6.17)-(6.19) we define the nonlinear operator $\mathbf{G} \equiv\left[G, G_{w}, U,\left\{W_{q}^{l}\right\}_{l=1}^{N_{P}},\left\{W_{Q}^{l}\right\}_{l=1}^{N_{I}}, F\right]$ where

$$
\begin{array}{rc}
G & \equiv c(s) \frac{\partial p}{\partial t}+\nabla \cdot \mathbf{u}-\sum_{l=1}^{N_{p}} q^{l} \delta\left(x-x_{P}^{l}\right)-\sum_{l=1}^{N_{I}} Q^{l} \delta\left(x-x_{I}^{l}\right) \\
G_{w} & \equiv \phi\left(c_{r}+c_{w}\right) s \frac{\partial p}{\partial t}+\phi \frac{\partial s}{\partial t}+\nabla \cdot f_{w} \mathbf{u}-\sum_{l=1}^{N_{p}} f_{w} q^{l} \delta\left(x-x_{P}^{l}\right)-\sum_{l=1}^{N_{I}} Q^{l} \delta\left(x-x_{I}^{l}\right),(6.26)
\end{array}
$$




$$
\begin{aligned}
U & \equiv \mathbf{u}+\lambda e^{Y} \nabla p \\
W_{q}^{l} & \equiv q^{l}-\omega_{P}^{l} K \lambda\left[P_{b h}^{l}-p\left(x_{P}^{l}\right)\right], \\
W_{Q}^{l} & \equiv Q^{l}-\omega_{I}^{l} K \lambda\left[p_{b h}^{l}-p\left(x_{I}^{l}\right)\right], \\
F & =f_{w}(s)-\frac{\lambda_{w}}{\lambda} .
\end{aligned}
$$

For the inverse problem we suppose that the following parameters are given:

$$
c_{\alpha}, c_{r}, s_{i w}, s_{o r}, a_{w}, a_{o},\left\{Q^{l}\right\}_{l=1}^{N_{I}},\left\{P_{b h}^{l}\right\}_{l=1}^{N_{P}}, \omega_{I}^{l}, \omega_{P}^{l},
$$

and the uncertain parameters are

$$
\mathcal{P} \equiv\left[Y=\log (K), \phi_{r}, \theta_{w}, \theta_{o}\right]
$$

Therefore, if we define the vector of state variables

$$
\mathcal{S} \equiv\left[s, p, \mathbf{u}, q^{l}, p_{b h}^{l}\right]
$$

the forward model can be written as

$$
\mathbf{G}(\mathcal{P}, \mathcal{S})=0
$$

\subsection{Data and Measurement Functionals}

We now discuss different type of measurements that will be assimilated with the IRBS.

1) Injection Wells. For the $l$-th injection well, $l \in\left\{1, \ldots, N_{I}\right\}$, we assume the bottomhole pressure (BHP) is measured at $M_{I}^{l}$ measurement times denoted by $\left\{t_{I}^{l, m}\right\}_{m=1}^{M_{I}^{l}}$. The vector of measurements is denoted by

$$
\mathbf{d}_{I}^{l} \equiv\left[d_{I}^{l, 1}, \ldots, d_{I}^{l, M_{I}^{l}}\right]^{T}, \quad l \in\left\{1, \ldots, N_{I}\right\}
$$

and the associated measurement functional on $p_{b h}^{l}(6.8)$ is defined by

$$
\mathcal{L}_{I}^{l, m}\left(p_{b h}^{l}\right) \equiv \int_{0}^{T} p_{b h}^{l}(t) \delta\left(t-t_{I}^{l, m}\right)
$$

Then, we define

$$
\mathcal{L}_{I}^{l} \equiv\left[\mathcal{L}_{I}^{l, 1}\left(p_{b h}^{l}\right), \ldots, \mathcal{L}_{I}^{l, M_{I}^{l}}\left(p_{b h}^{l}\right)\right]^{T}, \quad l \in\left\{1, \ldots, N_{I}\right\}
$$


2) Production Wells. For the $l$-th production well, $l \in\left\{1, \ldots, N_{p}\right\}$, we may consider measurements of the total flow rate as well as the fractional flow. Let $M_{P}^{l}$ be the number of measurement times denoted by $\left\{t_{P}^{l, m}\right\}_{m=1}^{M_{P}^{l}}$. In this case, the vector of measurements is defined by

$$
\mathbf{d}_{p}^{l} \equiv\left[d_{p}^{l, 1}, \ldots, d_{p}^{l, M_{P}^{l}}\right]^{T}, \quad l \in\left\{1, \ldots, N_{P}\right\}
$$

Furthermore, we consider a measurement functional defined by

$$
\mathcal{L}_{P}^{l, m}\left(q^{l}, f_{w}\left(x_{P}^{l}, t\right)\right)(t)=\int_{0}^{T}\left[a q^{l}(t)+b f_{w}\left(x_{P}^{l}, t\right)\right] \delta\left(t-t_{P}^{l, m}\right)
$$

for $a, b \in\{0,1\}$. We also define

$$
\mathcal{L}_{p}^{l} \equiv\left[\mathcal{L}_{p}^{l, 1}\left(q^{l}, f_{w}\left(x_{P}^{l}, t\right)\right), \ldots, \mathcal{L}_{p}^{l, M_{p}^{l}}\left(q^{l}, f_{w}\left(x_{P}^{l}, t\right)\right)\right]^{T}, \quad l \in\left\{1, \ldots, N_{p}\right\}
$$

3) Observation Wells. We finally consider observational pressure wells whose locations are denoted by $\left\{x_{o}^{l}\right\}_{l=1}^{N_{o}}$. For the $l$-th pressure-observation well, $l \in\left\{1, \ldots, N_{o}\right\}$, we assume there are $M_{o}^{l}$ measurement times denoted by $t_{o}^{l, m}$. The following variables are defined

$$
\begin{gathered}
\mathbf{d}_{o}^{l}=\left[d_{o}^{l, 1}, \ldots, d_{o}^{l, M_{o}^{l}}\right]^{T}, \quad l \in\left\{1, \ldots, N_{o}\right\}, \\
\mathcal{L}_{o}^{l, m}(p)(t)=\int_{\Omega} \int_{0}^{T} p(x, t) \delta\left(t-t_{o}^{l, m}\right) \delta\left(x-x_{o}^{l, m}\right), \\
\mathcal{L}_{o}^{l}=\left[\mathcal{L}_{o}^{l, 1}(p), \ldots, \mathcal{L}_{o}^{l, M_{o}^{l}}(p)\right]^{T}, \quad l \in\left\{1, \ldots, N_{o}\right\} .
\end{gathered}
$$

In general, for any index $\chi \in\{p, I, o\}$, the vector of observations can be written as

$$
\mathbf{d}_{\chi}^{l}=\left[d_{\chi}^{l, 1}, \ldots, d_{o}^{l, M_{\chi}^{l}}\right]^{T}, \quad l \in\left\{1, \ldots, N_{\chi}\right\}
$$


and a similar relation holds for $\mathcal{L}_{\chi}^{l}$. Then, let us define vectors

$$
\mathbf{d}_{\chi}=\left[\begin{array}{c}
\mathbf{d}_{\chi}^{1} \\
\vdots \\
\mathbf{d}_{\chi}^{N_{\chi}}
\end{array}\right], \quad \mathcal{L}_{\chi}=\left[\begin{array}{c}
\mathcal{L}_{\chi}^{1} \\
\vdots \\
\mathcal{L}_{\chi}^{N_{\chi}}
\end{array}\right]
$$

of dimension $\sum_{l=1}^{N_{\chi}} M_{\chi}^{l}$. In addition, for each type of measurement $\chi \in\{p, I, o\}$, at well $l \in\left\{1, \ldots, N_{\chi}\right\}$, we assume that the set of measurements $\mathbf{d}_{\chi}^{l}=\left\{d_{\chi}^{l, m}\right\}_{m=1}^{M_{\chi}^{l}}$ has an error covariance matrix denoted by $\mathbf{C}_{\chi, l}$. Finally, we define

$$
\mathbf{d}=\left[\begin{array}{c}
\mathbf{d}_{p} \\
\mathbf{d}_{I} \\
\mathbf{d}_{o}
\end{array}\right], \quad \mathcal{L}=\left[\begin{array}{c}
\mathcal{L}_{p} \\
\mathcal{L}_{I} \\
\mathcal{L}_{o}
\end{array}\right]
$$

\subsection{The IRBS for Two-Phase flow}

For the forward model defined in (6.31) and the assimilation information described above, we now present the IRBS for the identification of $\mathcal{P}$.

Algorithm 4 (IRBS for Two-Phase flow). We start with an initial guess $\overline{\mathcal{P}}$, as well as the corresponding prior error covariances $C_{Y}, C_{\phi}, \sigma_{w}$ and $\sigma_{o}$. For each $n=1, \ldots$,

(1) Solution to the forward model. Given the parameters estimated from the previous iteration $\mathcal{P}^{n-1} \equiv\left[Y^{n-1}, \phi_{r}^{n-1}, \theta_{w}^{n-1}, \theta_{o}^{n-1}\right]$, we compute the solution to the forward model $\mathcal{S}_{F}^{n}$, i.e, we solve

$$
G\left(\mathcal{P}^{n-1}, \mathcal{S}_{F}^{n}\right)=0
$$

where $\mathcal{S}_{F}^{n} \equiv\left(s_{F}^{n}, p_{F}^{n}, \mathbf{u}_{F}^{n}, q_{F}^{l, n}, p_{b h, F}^{l, n}\right)$.

(2) Stopping rule (Discrepancy Principle). If $\left\|\mathcal{D}^{\eta}-\mathcal{L}\left(\mathcal{S}_{F}^{n}\right)\right\| \leq \tau \eta$, stop. Output: $\mathcal{P}^{n-1}$.

(3) Parameter update by means of representers. Define $\mathcal{P}^{n}$ as the solution to the 
linearized data assimilation problem

$$
\begin{array}{r}
J=J_{d}+\int_{\Omega} \int_{\Omega}\left(Y-Y^{n-1}\right) C_{Y}^{-1}(x, y)\left(Y-Y^{n-1}\right) \\
+\int_{\Omega} \int_{\Omega}\left(\phi_{r}-\phi_{r}^{n-1}\right) C_{\phi}^{-1}(x, y)\left(\phi_{r}-\phi_{r}^{n-1}\right)+\sum_{\alpha} \frac{1}{\sigma_{\alpha}^{2}}\left(\theta_{\alpha}-\theta_{\alpha}^{n-1}\right)^{2} \\
\rightarrow \text { min },
\end{array}
$$

where

$$
J_{d}=\sum_{\chi} \sum_{l=1}^{N_{\chi}^{l}}\left[\mathbf{d}_{\chi}^{l}-\mathcal{L}_{\chi}^{l}\right] \mathbf{C}_{\chi, l}^{-1}\left[\mathbf{d}_{\chi}^{l}-\mathcal{L}_{\chi}\right]
$$

subject to

$$
L \tilde{G}\left(\mathcal{P}^{n-1}, \mathcal{S}_{F}^{n}\right) \equiv D_{\mathcal{P}} G\left(\mathcal{P}^{n-1}, \mathcal{S}_{F}^{n}\right)\left[\mathcal{P}^{n}-\mathcal{P}^{n-1}\right]+D_{s} G\left(\mathcal{P}^{n-1}, \mathcal{S}_{F}^{n}\right)\left[\mathcal{S}^{n}-\mathcal{S}_{F}^{n}\right]=0(6.47)
$$

In the following sections we discuss the implementation of Step (3) in Algorithm 4.

\subsubsection{The Euler-Lagrange Equations}

In order to compute the solution to problem (6.45) we need to find the linearized operator $L \tilde{G}$. First, for any function $g$ of $\left(s, \theta_{w}, \theta_{o}, \phi_{r}\right)$, we define

$$
D_{\tau}^{k} g_{F}^{n} \equiv\left(D_{\tau}^{k} g\right)\left(s_{F}^{n}, p_{F}^{n}, \theta_{w}^{n-1}, \theta_{o}^{n-1}, \phi_{r}^{n-1}\right)
$$

for $\tau \in\left\{s, \theta_{w}, \theta_{o}\right\}$ and $k \in\{0\} \cup \mathbb{N}$. In Appendix B we provide some key steps to reduce the formulation in terms only of the linearized variables $\mathcal{S} \equiv(p, s)$. Moreover, the linearized problem $L \tilde{G}=0$ is equivalent to

$$
\mathcal{L} \mathcal{G}\left[\mathcal{P}^{n}-\mathcal{P}^{n-1}, \mathcal{S}^{n}-\mathcal{S}_{F}^{n}\right]=0
$$

where

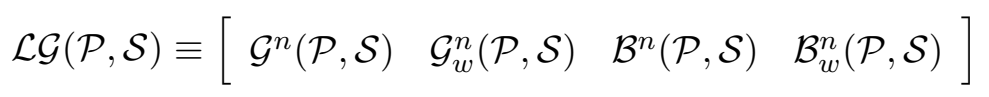


for

$$
\begin{gathered}
\mathcal{G}^{n}(\mathcal{P}, \mathcal{S}) \equiv \phi_{F}^{n} c\left(s_{F}^{n}\right) \frac{\partial p}{\partial t}+\phi_{F}^{n}\left[c_{w}-c_{o}\right] \frac{\partial p_{F}^{n}}{\partial t} s+\phi_{r} c\left(s_{F}^{n}\right) c_{r} \frac{\partial p_{F}^{n}}{\partial t} \\
+\nabla \cdot\left[\mathcal{T}^{n}(\mathcal{P}, \mathcal{S}) \mathbf{u}_{F}^{n}-\lambda_{F}^{n} e^{Y^{n-1}} \nabla p\right]-\sum_{l=1}^{N_{p}}\left[\mathcal{T}^{n}(\mathcal{P}, \mathcal{S}) q_{F}^{n}-\omega_{I}^{p, l} \lambda_{F}^{n} e^{Y^{n-1}} p\right] \delta\left(x-x_{p}^{l}\right) \\
\mathcal{G}_{w}^{n}(\mathcal{P}, \mathcal{S}) \equiv \phi_{F}^{n}\left(c_{r}+c_{w}\right) s_{F}^{n} \frac{\partial p}{\partial t}+c_{r} \phi_{r}^{n-1} p \frac{\partial s_{F}^{n}}{\partial t}+\phi_{F}^{n}\left(c_{r}+c_{w}\right) s \frac{\partial p_{F}^{n}}{\partial t} \\
+\phi_{F}^{n} \frac{\partial s}{\partial t}+\phi_{r}\left[\left(c_{r}+c_{w}\right) s_{F}^{n} \frac{\partial p_{F}^{n}}{\partial t}+\left[1+c_{r}\left[p_{F}^{n}-p_{r}\right]\right] \frac{\partial s_{F}^{n}}{\partial t}\right] \\
+\nabla \cdot\left[\mathcal{T}_{w}^{n}(\mathcal{P}, \mathcal{S}) \mathbf{u}_{F}^{n}-\lambda_{w, F}^{n} e^{Y^{n-1}} \nabla p\right] \\
-\sum_{l=1}^{N_{p}}\left[\mathcal{T}_{w}^{n}(\mathcal{P}, \mathcal{S}) q_{F}^{n}-\omega_{I}^{p, l} \lambda_{w, F}^{n} e^{Y^{n-1}} p\right] \delta\left(x-x_{p}^{l}\right) \\
\mathcal{B}^{n} \equiv\left[\mathcal{T}^{n}(\mathcal{P}, \mathcal{S}) \mathbf{u}_{F}^{n}-\lambda_{F}^{n} e^{Y^{n-1}} \nabla p\right] \cdot \mathbf{n}, \\
\mathcal{B}_{w}^{n} \equiv\left[\mathcal{T}_{w}^{n}(\mathcal{P}, \mathcal{S}) \mathbf{u}_{F}^{n}-\lambda_{w, F}^{n} e^{Y^{n-1}} \nabla p\right] \cdot \mathbf{n}
\end{gathered}
$$

and the auxiliary operators

$$
\begin{aligned}
& \mathcal{T}^{n}(\mathcal{P}, \mathcal{S}) \equiv \kappa_{s}^{n} s+Y+\sum_{\alpha} \kappa_{w}^{n} \theta_{\alpha} \\
& \mathcal{T}_{w}^{n}(\mathcal{P}, \mathcal{S}) \equiv \kappa_{w, s}^{n} s+f_{w} Y+\kappa_{w}^{n} \theta_{w}
\end{aligned}
$$

In (6.51)-(6.56), we have applied the following formulas

$$
\begin{gathered}
\kappa_{s}^{n}=\frac{D_{s} \lambda_{F}^{n}}{\lambda_{F}^{n}}, \quad \kappa_{\alpha}^{n}=\frac{D_{\theta_{\alpha}} \lambda_{F}^{n}}{\lambda_{F}^{n}}, \quad \kappa_{w, s}^{n}=\frac{D_{s} \lambda_{w, F}^{n}}{\lambda_{F}^{n}}, \\
\phi_{F}^{n}=\phi_{r}^{n-1}\left[1+c_{r}\left(p_{F}^{n}-p_{r}\right)\right],
\end{gathered}
$$

defined according to (6.48). Note that all variables with superscript $n$ and/or subscript $F$ are known at the current iteration.

As we discussed in Chapter (4), problem (6.45) can be solved by computing the 
solution to the EL system that arises from the problem

$$
\mathcal{Q} \rightarrow \min
$$

where

$$
\begin{array}{r}
\mathcal{Q}=J+\int_{\Omega} \int_{0}^{T}\left[\Lambda \mathcal{G}^{n}+\Lambda_{w} \mathcal{G}_{w}^{n}\right]-\int_{\Gamma} \int_{0}^{T}\left[\Lambda \mathcal{B}^{n}+\Lambda_{w} \mathcal{B}_{w}^{n}\right] \\
+\int_{\Omega}\left[\Lambda \phi_{F}^{n} c\left(s_{F}^{n}\right)\left(p-p_{F}^{n}\right)+\Lambda_{w}\left[\phi_{F}^{n}\left(c_{r}+c_{w}\right) s_{F}^{n}\left(p-p_{F}^{n}\right)+\phi_{F}^{n}\left(s-s_{F}^{n}\right)\right]\right]_{t=0}
\end{array}
$$

Note that in (6.59), $\Lambda$ and $\Lambda_{w}$ are the Lagrange multipliers associated with the two constraint functions (6.51)-(6.52). Some aspects of (6.58)-(6.59) are now pointed out. For each $\chi \in\{p, I, o\}, l \in\left\{1, \ldots, N_{\chi}\right\}$, and $i \in\{1, \ldots, 5\}$ we define vectors $\mathbf{D}_{i, \chi}^{l} \in \mathbb{R}^{M_{\chi}^{l}}$ by

$$
D_{i, \chi}^{l, m}=X_{i, \chi}^{l, m} \delta\left(x-x_{\chi}^{l}\right) \delta\left(t-t_{\chi}^{l, m}\right), \quad m \in\left\{1, \ldots, M_{\chi}^{l}\right\}
$$

where

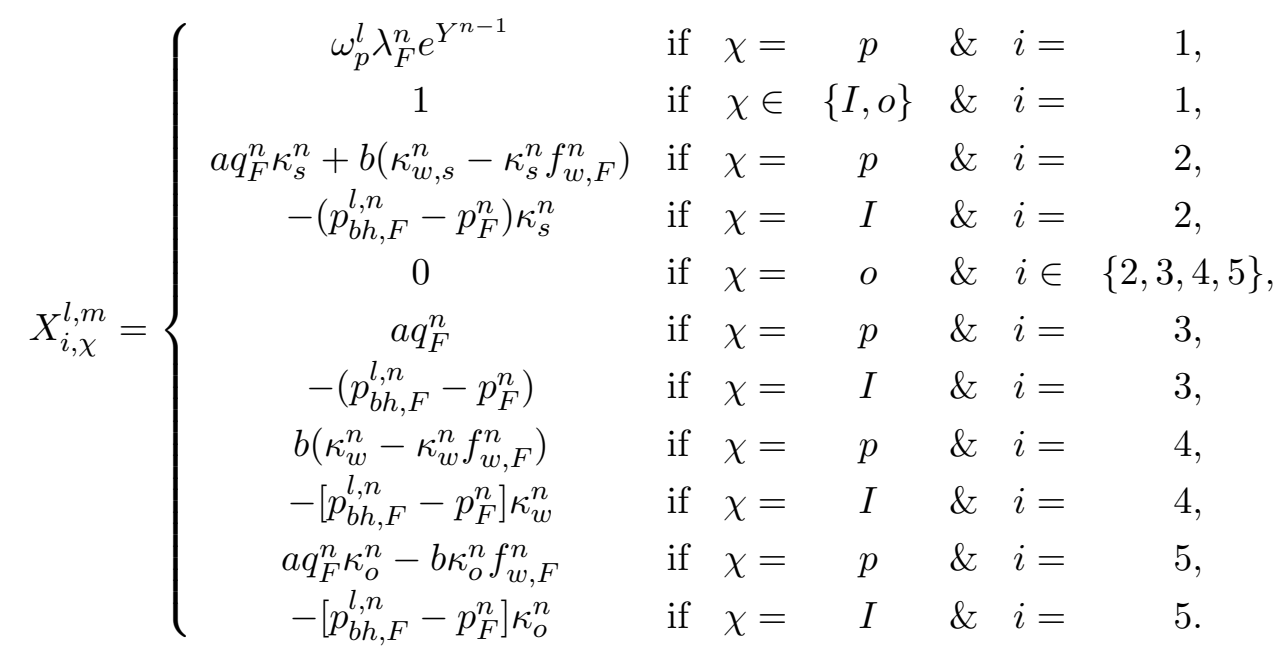

In Appendix B we show that the EL equations for problem (6.59) are (6.49) as well as the following

\section{Adjoint System}

$$
\begin{aligned}
\mathcal{A}^{n}\left[\Lambda, \Lambda_{w}\right] & =\sum_{\chi} \sum_{l=1}^{N_{\chi}}\left[\mathbf{d}_{\chi}^{l}-\mathcal{L}_{\chi}^{l}\right] \mathbf{C}_{I, l}^{-1} \mathbf{D}_{1, \chi}^{l} \\
\mathcal{A}_{w}^{n}\left[\Lambda, \Lambda_{w}\right], & =\sum_{\chi} \sum_{l=1}^{N_{\chi}}\left[\mathbf{d}_{\chi}^{l}-\mathcal{L}_{\chi}^{l}\right] \mathbf{C}_{I, l}^{-1} \mathbf{D}_{2, \chi}^{l}
\end{aligned}
$$


where

$$
\begin{array}{r}
\mathcal{A}^{n}\left[\Lambda, \Lambda_{w}\right]=-\frac{\partial}{\partial t}\left[\Lambda \phi_{F}^{n} c\left(s_{F}^{n}\right)\right]-\frac{\partial}{\partial t}\left[\Lambda_{w} \phi_{F}^{n}\left(c_{r}+c_{w}\right) s_{F}^{n}\right]+\Lambda_{w} c_{r} \phi_{r}^{n-1} \frac{\partial s_{F}^{n}}{\partial t} \\
-\nabla \cdot\left[\lambda_{F}^{n} e^{Y^{n-1}} \nabla \Lambda+\lambda_{w, F}^{n} e^{Y^{n-1}} \nabla \Lambda_{w}\right]+\sum_{l=1}^{N_{p}} \omega_{I}^{p, l}\left[\lambda_{F}^{n} e^{Y^{n-1}} \Lambda+\lambda_{w, F}^{n} e^{Y^{n-1}} \Lambda_{w}\right] \delta\left(x-x_{p}^{l}\right)
\end{array}
$$

and

$$
\begin{array}{r}
\mathcal{A}_{w}^{n}\left[\Lambda, \Lambda_{w}\right] \equiv \Lambda \phi_{F}^{n}\left[c_{w}-c_{o}\right] \frac{\partial p_{F}^{n}}{\partial t}+\Lambda_{w} \phi_{F}^{n}\left(c_{r}+c_{w}\right) \frac{\partial p_{F}^{n}}{\partial t}-\frac{\partial\left[\phi_{F}^{n} \Lambda_{w}\right]}{\partial t} \\
-\nabla \cdot\left[\left(\Lambda \kappa_{s}^{n}+\Lambda_{w} \kappa_{w, s}^{n}\right) \mathbf{u}_{F}^{n}\right]+\Lambda \nabla \cdot\left[\kappa_{s}^{n} \mathbf{u}_{F}^{n}\right]+\Lambda_{w} \nabla \cdot\left[\kappa_{w, s}^{n} \mathbf{u}_{F}^{n}\right] \\
-\sum_{l=1}^{N_{p}} q_{F}^{n}\left[\Lambda q_{F}^{n} \kappa_{s}^{n}+\Lambda_{w} \kappa_{w, s}^{n}\right] \delta\left(x-x_{p}^{l}\right) .
\end{array}
$$

System (6.62)-(6.63) is supplied with homogeneous Neummann boundary conditions and final time conditions for $\Lambda$ and $\Lambda_{w}$.

\section{Control Equations}

The control equations for $\mathcal{P}$ are

$$
\begin{aligned}
Y=Y^{n-1}+\int_{\Omega} \int_{0}^{T} C_{Y}^{-1}(x, y)\left[\left[\nabla \Lambda+f_{w, F}^{n} \nabla \Lambda_{w}\right] \cdot \mathbf{u}_{F}^{n}\right. & +\sum_{l=1}^{N_{p}} q_{F}^{n}\left[\Lambda+f_{w, F}^{n} \Lambda_{w}\right] \delta\left(x-x_{p}^{l}\right) \\
& \left.+\sum_{\chi} \sum_{l=1}^{N_{\chi}}\left[\mathbf{d}_{\chi}^{l}-\mathcal{L}_{\chi}^{l}\right] C_{\chi, l}^{-1} \mathbf{D}_{3, \chi}^{l}\right],(6 \\
\theta_{w}=\theta_{w}^{n-1}+\sigma_{w}^{2} \int_{\Omega} \int_{0}^{T}\left[\kappa_{w}^{n} \nabla\left[\Lambda+\Lambda_{w}\right] \cdot \mathbf{u}_{F}^{n}+\right. & \sum_{l=1}^{N_{p}} q_{F}^{n} \kappa_{w}^{n}\left[\Lambda+\Lambda_{w}\right] \delta\left(x-x_{P}^{l}\right) \\
& \left.+\sum_{\chi} \sum_{l=1}^{N_{\chi}}\left[\mathbf{d}_{\chi}^{l}-\mathcal{L}_{\chi}^{l}\right] C_{\chi, l}^{-1} \mathbf{D}_{4, \chi}^{l}\right]
\end{aligned}
$$




$$
\begin{gathered}
\theta_{o}=\theta_{o}^{n-1}+\sigma_{o}^{2} \int_{\Omega} \int_{0}^{T}\left[\kappa_{o}^{n} \nabla \Lambda \cdot \mathbf{u}_{F}^{n}+\sum_{l=1}^{N_{p}} q_{F}^{n} \kappa_{o}^{n} \Lambda \delta\left(x-x_{p}^{l}\right)+\sum_{\chi} \sum_{l=1}^{N_{\chi}}\left[\mathbf{d}_{\chi}^{l}-\mathcal{L}_{\chi}^{l}\right] C_{\chi, l}^{-1} \mathbf{D}_{5, \chi}^{l}\right] \\
\phi_{r}=\phi_{r}^{n-1}-\int_{0}^{T} \int_{\Omega} C_{\phi}^{-1}(x, y)\left[\Lambda c\left(s_{F}^{n}\right) \frac{\partial p_{F}^{n}}{\partial t}+\frac{\Lambda_{w}}{\phi_{r}^{n-1}}\left[\phi_{r}^{n-1}\left(c_{r}+c_{w}\right) s_{F}^{n} \frac{\partial p_{F}^{n}}{\partial t}+\phi_{F}^{n} \frac{\partial s_{F}^{n}}{\partial t}\right]\right]
\end{gathered}
$$

In the following section, equations (6.49), (6.62)-(6.69) will be solved by representer expansions. From Chapter (3) we know that any state variable and parameter can be written as a linear combination of a representer function that contributes for each measurement utilized for the inversion. For this reason we postulate that for $\xi \in\left\{p, s, Y, \phi_{r}, \theta_{w}, \theta_{o}\right\}$, the following expansion holds

$$
\xi=\xi_{F}^{n}+\sum_{\chi \in\{p, I, o\}} \sum_{l=1}^{N_{\chi}} \sum_{j=1}^{M_{\chi}^{l}} \beta_{\chi}^{l, j} R_{\chi, \xi}^{l, j}
$$

\subsubsection{Definitions and Notation}

We define

$$
\mathcal{B}\left[s, \theta_{w}, \theta_{o}\right]=\left[\kappa_{w, s}^{n}-\kappa_{s}^{n} f_{w, F}^{n}\right] s+\left[\kappa_{w}^{n}-\kappa_{w}^{n} f_{w, F}^{n}\right] \theta_{w}-\kappa_{o}^{n} f_{w, F}^{n} \theta_{o}
$$

Furthermore, for each $\chi \in\{p, I, o\}$, every $l \in\left\{1 \ldots, N_{\chi}\right\}$ and every $j \in\left\{1 \ldots, M_{\chi}^{k}\right\}$, we define

$$
\begin{gathered}
\mathbf{r}_{\chi}^{l, j} \equiv\left(R_{\chi, p}^{l, j}, R_{\chi, s}^{l, j}, R_{\chi, Y}^{l, j}, R_{\chi, w}^{l, j}, R_{\chi, o}^{l, j}\right), \\
\mathbf{v}_{\chi}^{l, j} \equiv\left(R_{\chi, s}^{l, j}, R_{\chi, w}^{l, j}, R_{\chi, o}^{l, j}\right) .
\end{gathered}
$$

For each $\xi \in\{p, I, o\}$, every $k \in\left\{1 \ldots, N_{\xi}\right\}$ and every $m \in\left\{1 \ldots, M_{\xi}^{k}\right\}$ let us define

$$
\left[\tau_{\xi, \chi}^{k, m, l}\right]_{j} \equiv\left\{\begin{array}{cl}
\int_{0}^{T} \delta\left(t-t_{p}^{l, m}\right)\left[a q\left[q_{F}^{n} \mathcal{T}\left[\mathbf{r}_{\chi}^{l, j}\right]-\omega_{p}^{k} \lambda_{F}^{n} e^{Y^{n-1}} R_{\chi, p}^{l, j}\right]+b \mathcal{B}[\mathbf{v} \chi, j]\right. & \text { if } \xi=p \\
-\int_{0}^{T} \delta\left(t-t_{I}^{k, m}\right)\left[\left[p_{b h}^{l, n}-p_{F}^{n}\right] \mathcal{T}\left[\mathbf{r}_{j}^{\chi, l}\right]-R_{\chi, p}^{l, j}\right] & \text { if } \xi=I \\
\int_{0}^{T} \delta\left(t-t_{o}^{k, m}\right) R_{\chi, p}^{l, j} & \text { if } \xi=o
\end{array}\right.
$$


where $\chi \in\{p, I, o\}, l \in\left\{1, \ldots, N_{\chi}\right\}$ and $j \in\left\{1, \ldots, M_{\chi}^{l}\right\}$. We also define vector

$$
\boldsymbol{\tau}_{\xi, \chi}^{k, m, l} \equiv\left(\left[\tau_{\xi, \chi}^{k, m, l}\right]_{1}, \ldots,\left[\tau_{\xi, \chi}^{k, m, l}\right]_{M_{\chi}^{l}}\right)
$$

and matrix

$$
\boldsymbol{v}_{\xi, \chi}^{k, l}=\left[\begin{array}{c}
\boldsymbol{\tau}_{\xi, \chi}^{k, 1, l} \\
\vdots \\
\boldsymbol{\tau}_{\xi, \chi}^{k, M_{\xi}^{k}, l}
\end{array}\right]
$$

Note that $\boldsymbol{\tau}_{\xi, \chi}^{k, m, l} \in \mathbb{R}^{M_{\chi}^{l}}$ and $\boldsymbol{v}_{\xi, \chi}^{k, l} \in \mathbb{R}^{M_{\xi}^{k} \times M_{\chi}^{l}}$. Additionally,

$$
\mathbf{V}_{\xi, \chi}^{k, l} \equiv\left\{\begin{array}{cc}
\mathbf{C}_{\chi, k}+\boldsymbol{v}_{\xi, \chi}^{k, l} & \text { if } k=l \text { and } \chi=\xi \\
\boldsymbol{v}_{\xi, \chi}^{k, l} & \text { otherwise, }
\end{array}\right.
$$

where $\mathbf{C}_{\chi, k}$ is the covariance matrix introduced in Section (6.2). Furthermore, let us define

$$
\mathbf{V}_{\xi, \chi}^{l}=\left[\begin{array}{c}
\mathbf{V}_{\xi, \chi}^{1, l} \\
\vdots \\
\mathbf{V}_{\xi, \chi}^{N_{\xi}, l}
\end{array}\right], \quad \mathbf{b}_{\chi} \equiv\left[\begin{array}{c}
\mathbf{b}_{\chi}^{1} \\
\vdots \\
\mathbf{b}_{\chi}^{N_{\chi}}
\end{array}\right]
$$

where

$$
\mathbf{b}_{\chi}^{l} \equiv\left(\beta_{\chi}^{l, 1}, \ldots, \beta_{\chi}^{l, M_{\chi}^{l}}\right)
$$

Note that $\mathbf{b}_{\chi} \in \mathbb{R}^{\sum_{l=1}^{N_{\chi}^{l}} M_{\chi}^{l}}$ and $\mathbf{V}_{\xi, \chi}^{l} \in \mathbb{R}^{\sum_{k=1}^{N_{\xi}} M_{\xi}^{k} \times M_{\chi}^{l}}$. Finally, we define matrix $\mathbf{V}_{\xi, \chi} \in$ $\mathbb{R}^{\sum_{k=1}^{N_{\xi}} M_{\xi}^{k} \times \sum_{l=1}^{N_{\chi}} M_{\chi}^{l}}$ by the following relation

$$
\mathbf{V}_{\xi, \chi} \equiv\left[\begin{array}{lll}
\mathbf{V}_{\xi, \chi}^{1} & \cdots & \mathbf{V}_{\xi, \chi}^{N_{\chi}}
\end{array}\right]
$$

\subsection{Implementation}

For the adjoint (6.62)-(6.64) we consider the following expression

$$
\begin{aligned}
\Lambda & =\sum_{\chi \in\{p, I, o\}} \sum_{l=1}^{N_{\chi}} \sum_{j=1}^{M_{\chi}^{l}} \beta_{\chi}^{l, j} \Lambda_{\chi}^{l, j}, \\
\Lambda_{w} & =\sum_{\chi \in\{p, I, o\}} \sum_{l=1}^{N_{\chi}} \sum_{j=1}^{M_{\chi}^{l}} \beta_{\chi}^{l, j} \Lambda_{\chi, w}^{l, j} .
\end{aligned}
$$


When (6.70) and (6.83)-(6.84) are substituted in (6.49), (6.62)-(6.69) we obtain the following algorithm which is Step (3) of Algorithm 4.

Algorithm 5 (Step (3) of Algorithm 4). For each $\chi \in\{p, I, o\}, l \in\left\{1, \ldots, N_{\chi}^{l}\right\}$ and $j \in\left\{1, \ldots, M_{\chi}^{l}\right\}$

(1) Find the adjoint representers $\Lambda_{\chi}^{l, j}$ and $\Lambda_{\chi, w}^{l, j}$ by solving

$$
\begin{gathered}
\mathcal{A}\left[\Lambda_{\chi}^{l, j}, \Lambda_{\chi, w}^{l, j}\right]=D_{1, \chi}^{l, j} \\
\mathcal{A}_{w}\left[\Lambda_{\chi}^{l, j}, \Lambda_{\chi, w}^{l, j}\right]=D_{2, \chi}^{l, j} .
\end{gathered}
$$

(2) Compute the permeability, relative permeabilities and porosity representers with

$$
\begin{gathered}
R_{\chi, Y}^{l, j}=\int_{\Omega} \int_{0}^{T} C_{Y}^{-1}(x, y)\left[\nabla \Lambda_{\chi}^{l, j}+f_{w, F}^{n} \nabla \Lambda_{\chi, w}^{l, j} \cdot \mathbf{u}_{F}^{n}+\sum_{l=1}^{N_{p}} q_{F}^{n}\left[\Lambda_{\chi}^{l, j}+f_{w, F}^{n} \Lambda_{\chi, w}^{l, j}\right] \delta\left(x-x_{p}^{l}\right)+D_{3, \chi}^{l, j}\right] \\
R_{\chi, w}^{l, j}=\int_{\Omega} \int_{0}^{T}\left[\kappa_{w}^{n} \nabla\left[\Lambda_{\chi}^{l, j}+\Lambda_{\chi, w}^{l, j}\right] \cdot \mathbf{u}_{F}^{n}+\sum_{l=1}^{N_{p}} q_{F}^{n} \kappa_{w}^{n}\left[\Lambda_{\chi}^{l, j}+\Lambda_{\chi, w}^{l, j}\right] \delta\left(x-x_{p}^{l}\right)+D_{4, \chi}^{l, j}\right] \\
R_{\chi, o}^{l, j}=\int_{\Omega} \int_{0}^{T}\left[\kappa_{o}^{n} \nabla \Lambda_{\chi}^{l, j} \cdot \mathbf{v}_{F}^{n}+\sum_{l=1}^{N_{p}} q_{F}^{n} \tau_{o}^{n} \Lambda_{\chi}^{l, j} \delta\left(x-x_{p}^{l}\right)+D_{5, \chi}^{l, j}\right]
\end{gathered}
$$

and

$$
R_{j, \phi}^{\chi, l}=-\int_{0}^{T} \int_{\Omega} C_{\phi}^{-1}(x, y)\left[A_{j}^{\chi, l} c\left(s_{F}^{n}\right) \frac{\partial p_{F}^{n}}{\partial t}+\frac{A_{j, w}^{\chi, l}}{\phi_{r}^{n-1}}\left[\phi_{r}^{n-1}\left(c_{r}+c_{w}\right) s_{F}^{n} \frac{\partial p_{F}^{n}}{\partial t}+\phi_{F}^{n} \frac{\partial s_{F}^{n}}{\partial t}\right]\right] .
$$

(3) Compute the forward representers $R_{\chi, p}^{l, j}$ and $R_{\chi, s}^{l, j}$ from the following system

$$
\begin{aligned}
\mathcal{G}^{n}\left(R_{\chi, p}^{l, j}, R_{\chi, s}^{l, j}, R_{\chi, Y}^{l, j}, R_{\chi, w}^{l, j}, R_{\chi, o}^{l, j}, R_{\chi, \phi_{r}}^{l, j}\right) & =0 \\
\mathcal{G}_{w}^{n}\left(, R_{\chi, p}^{l, j}, R_{\chi, s}^{l, j}, R_{\chi, Y}^{l, j}, R_{\chi, w}^{l, j}, R_{\chi, \phi_{r}}^{l, j}\right) & =0 .
\end{aligned}
$$


(4) Find the coefficients (6.70) $\beta_{\chi}^{j, l}$ from

$$
\mathrm{Vb}=\mathbf{d}-\mathcal{L}
$$

where $\mathbf{d}$ and $\mathcal{L}$ were defined in (6.43). Matrix $\mathbf{V}$ (respect. b) is a block matrix (vector) whose entry $(\xi, \chi)$ is matrix $\mathbf{V}_{\xi, \chi}\left(\mathbf{b}_{\xi}\right)$ defined by (6.82) (respect. (6.81)).

\subsection{Numerical Experiments. Waterflood}

In this section we present some numerical results of the implementation of Algorithm 4 (with Step (3) given by Algorithm 5) for the estimation of petrophysical properties in the model described by (6.31).

\subsubsection{Estimation of Absolute and Relative Permeabilities}

In Experiment $\mathrm{V}$ we apply the IRBS-KL to the joint estimation of absolute and relative permeabilities. We use Algorithm 4 assuming that porosity is known $\left(C_{\phi}=0\right)$. For the absolute permeability we choose the true permeability of Figure 6.1(b). This permeability is chosen from a set of 200 realizations consistent with the following semivariogram model

$$
\gamma=0.35 \operatorname{Sp}_{135}(1000,150)+0.35 \operatorname{Exp}_{90}(200,200)
$$

The first term of this expression denotes a contribution of $0.35 \mathrm{md}$ of a spherical variogram with maximum continuity at an azimuthal angle of 135 degrees (with respect to the northsouth direction). The maximum and minimum ranges of continuity are $1000 \mathrm{ft}$ and $150 \mathrm{ft}$ respectively. The second term corresponds to a contribution of $0.35 \mathrm{md}$ of an exponential isotropic variogram with range $200 \mathrm{ft}$ at an azimuthal angle of 90 degrees.

We use 180 realization (not including the true permeability) to perform the KL parametrization for log-permeability as described in Chapter 5. We retain 90 eigenvectors corresponding to the highest eigenvalues of the KL decomposition. The identification of absolute permeability is carried out for $\xi$ in expression (5.10). On the other hand, the estimation of the relative permeabilities is based on the exponent of curves (6.24)-(6.26). We assume "true parameters" $\theta_{w}=3.2$ and $\theta_{o}=2.8$. These parameters as well as the permeability of Figure 6.1 are used to generate synthetic data with the forward simulator with relevant data displayed in Tables 6.1. Well configuration as well as the prior error logpermeability are presented in Figure 6.1 (a). Pertinent information about data assimilation is displayed in Table 6.3. The estimated absolute permeability field is displayed in Figure 
6.1 (c). The estimated parameters of the relative permeability curves can be found in the last column of Table (6.2). In Figure 6.3 we show the relative permeability curves corresponding to the true, prior $(n=0)$ and estimated $(n=5)$ parameters. Additionally, history matching of wells are shown in Figure 6.2 and convergence performance is available in Table 6.2. Note that convergence is achieved first for BHP measurements $(n=4)$. In general, each parameter has different sensitivity to each measurement type. For this reason, the stopping rule (see Chapter 3) must be simultaneously satisfied by each type of observation misfit.

The results of this experiment are quite satisfactory. Improved estimates of the relative permeabilities were obtained. Moreover, the estimated absolute permeability not only captures the main heterogeneities but also the geological constraint. However, in the region near well I1 we observe that a poor identification was obtained. In contrast, the corresponding history matching plot (Figure 6.2 (a)) shows almost a perfect match to the measurement. When we look closely at I1, we see a good reconstruction only within a small (two or three gridblocks) neighborhood of I1. This situation can be attributed to expression (6.8) where the permeability is evaluated at the gridblock containing I1. Therefore, once the value of the log-permeability at that gridblock has been identified, no additional information can be learn from the observation at well I1. It is clear that further investigation of well models may improve the inverse computations.

TABLE 6.1: Reservoir description. Experiment $V$ and VI.

\begin{tabular}{lcc}
\hline Experiment & V and VI & VII \\
Dimension $\mathrm{ft}^{3}$ & $1315 \times 1315 \times 3.5$ & $2000 \times 7000 \times 32$ \\
Grid Blocks (for synthetic data) & $80 \times 80 \times 1$ & $60 \times 110 \times 1$ \\
Time interval $[0, T]$ (years) & {$[0,2.7]$} & {$[0,2.7]$} \\
Time discretization $d t$ (days) & 8.0 & 8.0 \\
Water compressibility ( $\left.\mathrm{psi}^{-1}\right)$ & $3.1 \mathrm{e}-6$ & $3.1 \mathrm{e}-6$ \\
Rock compressibility $\left(\mathrm{psi}^{-1}\right)$ & $1.0 \mathrm{e}-6$ & $1.0 \mathrm{e}-6$ \\
Water viscosity (cp) & 3.0 & 3.0 \\
Oil viscosity (cp) & 1.0 & 1.0 \\
Initial pressure (psi) & 6165 & 6236 \\
Number Injection Wells & 9 & 15 \\
Number Production Wells & 4 & 8 \\
${ }^{a}$ Injection rate (bbl/day) & 100 & 700 \\
${ }^{a}$ Production BHP (psi) & 6100 & 6160 \\
\hline
\end{tabular}

${ }^{a}$ We use constant BHP (rate) at every well production (injection) well. 


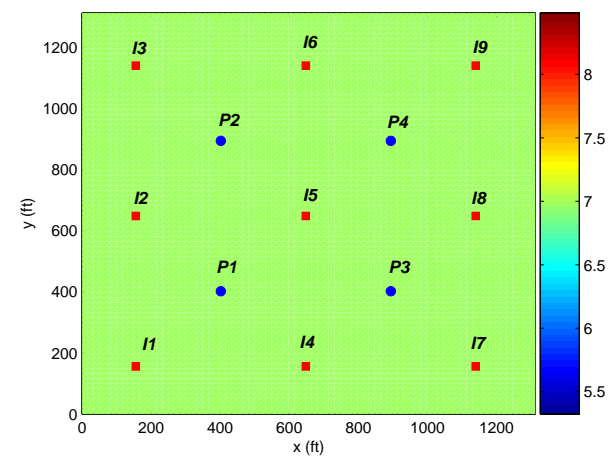

(A) Well Configuration and Prior $\ln (K)$

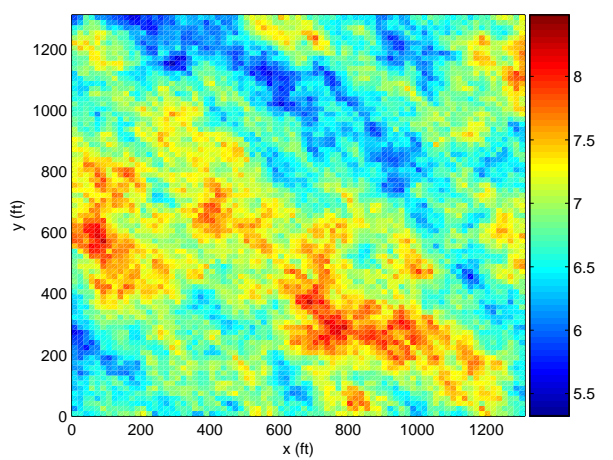

(B) True

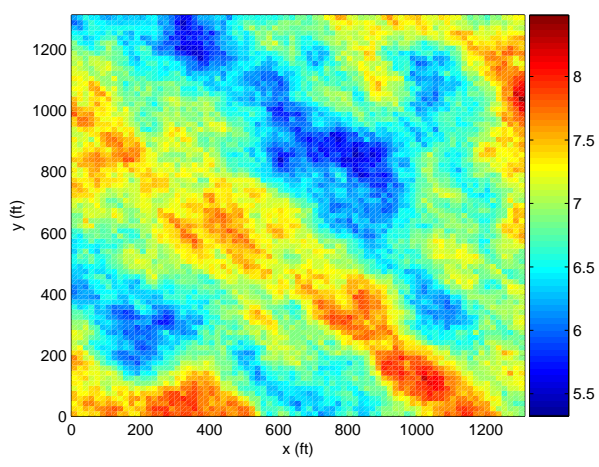

(c) $I R B S-K L$

FiguRE 6.1: Experiment V. Log permeability fields [n $m d]$.

\subsubsection{Estimation of Porosity}

In Experiment VI we consider the application of the IRBS-KL to the identification of porosity. This petrophysical property does not have a large range of variability. Then, from the identification point of view, we may think it is easy to identify. However, as we will see in this experiment there are interesting challenges to face.

We assume that absolute and relative permeabilities are known $\left(C_{Y}=0=\sigma_{w}=\sigma_{o}\right)$. A true porosity is generated from 180 unconditional realizations having an isotropic spherical covariance with range of $300 \mathrm{ft}$ and a contribution of 0.3 . The same process for KL parametrization was applied to parameterize the porosity field. For this experiment we assume all the parameters are perfectly known except the porosity. When we inspect equations (6.8) and conduct some experiments, we realize that bottom hole pressure collected at injection wells is not too sensitive to the changes in porosity. In contrast, a change in 


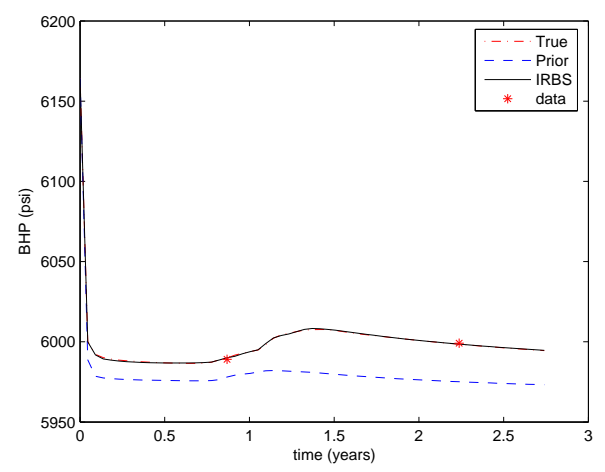

(A) $P H B($ Well I1)

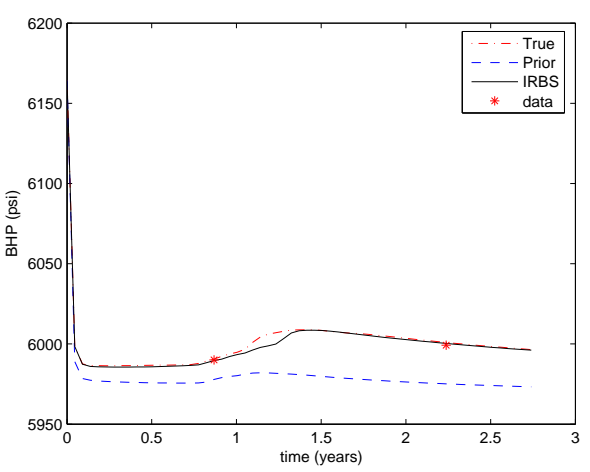

(c) PHB (Well I9)

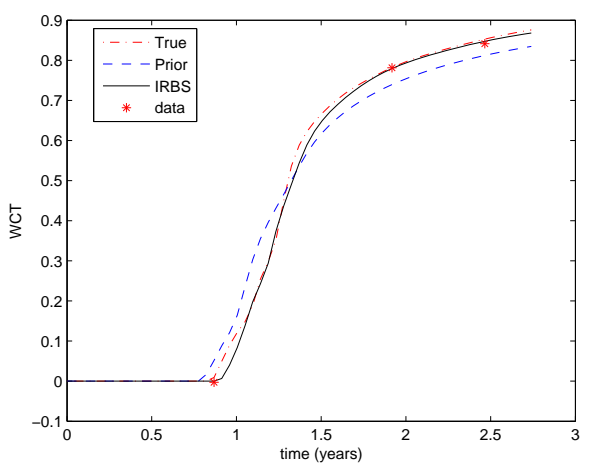

(E) WCT (Well P2)

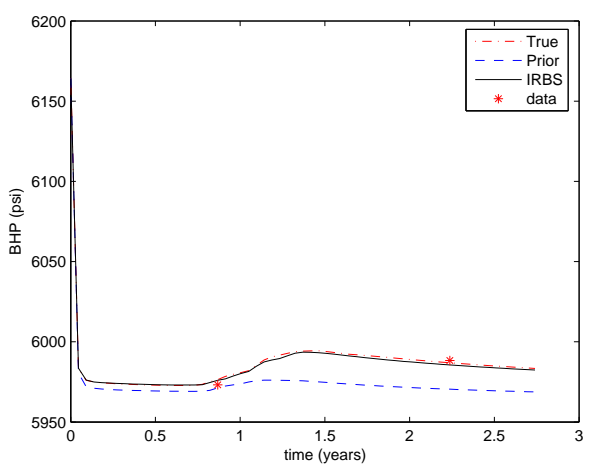

(B) PHB (Well I5)

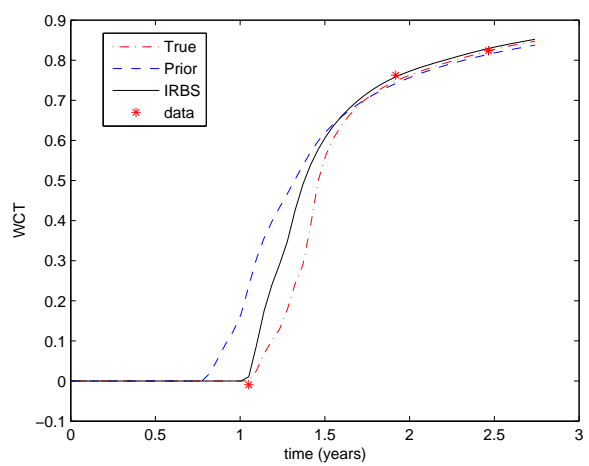

(D) WCT (Well P1)

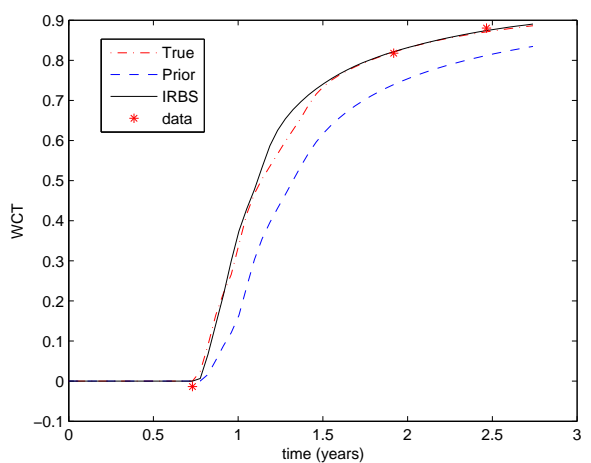

(F) WCT (Well P3)

FIgURE 6.2: Bottom hole pressure (BPP) and water cut (WCT) history-matching. Experiment $V$. 


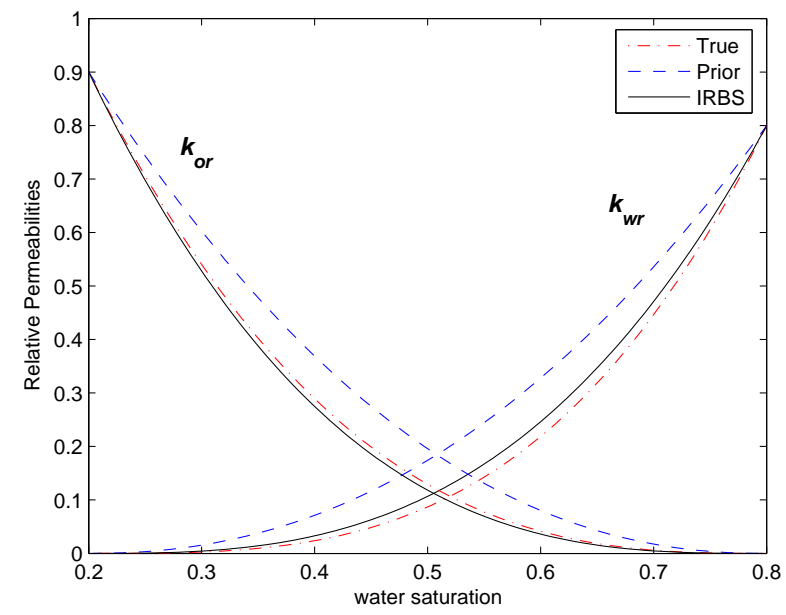

(A)

FiguRE 6.3: Experiment V. Relative permeability curves.

TABle 6.2: Convergence Results. Experiment $V$.

\begin{tabular}{lllll}
\hline (iteration) $n$ & $\left\|d_{p}^{\eta}-\mathcal{L}\left(f_{w, F}^{n}\right)\right\|_{C^{-1}}^{2}$ & $\left\|d_{I}^{\eta}-\mathcal{L}\left(p_{b h}\right)\right\|_{C^{-1}}^{2}$ & $\theta_{w}$ & $\theta_{o}$ \\
\hline 1 & $1.353 \mathrm{e} 3$ & $2.061 \mathrm{e} 3$ & 2.2 & 2.2 \\
2 & $4.982 \mathrm{e} 2$ & $4.376 \mathrm{e} 2$ & 3.1978 & 2.8516 \\
3 & $1.229 \mathrm{e} 2$ & $3.850 \mathrm{e} 1$ & 2.8653 & 2.4465 \\
4 & $2.07 \mathrm{e} 1$ & $1.33 \mathrm{e} 1$ & 2.9087 & 2.8897 \\
5 & $8.5 \mathrm{e} 0$ & $1.30 \mathrm{e} 1$ & 2.9053 & 2.9273 \\
\hline
\end{tabular}

porosity is reflected in the production wells water cut curves. This situation can be formally observed from the simple expression of the Buckley-Leverett equation as the velocity front is inversely proportional to porosity. Therefore, it comes as no surprise that only measurements from production wells after breaktrough contributes to the identification of porosity. The prior mean and well configuration is shown in Figure 6.4(a). In Figure 6.4(b) we present an estimate obtained with IRBS-KL for (signal-to-noise ratio) $S N R=700$ (Experiment VI(a)). Since we obtained a poor identification, it seems that this choice of noise level is small for a substantial identification of porosity. However, it is interesting to observe from the correspoding history matching curves (Figures 6.5,6.6 (A) and (C)) that the aforementioned SNR provides reasonable matched curves. When we repeat the experiment for $S N R=1 e 4$ (Experiment $\mathrm{VI}(\mathrm{b})$ ), the estimated porosity presented in Figure 6.4 (c), shows a considerable improvement. In this case, the history curves (Figures 6.5,6.6 (B) 
TABLE 6.3: Information for data assimilation. Experiment $V$.

\begin{tabular}{lll}
\hline Well type & Production & Injection \\
\hline Measurement type & water cut (WCT) & Bottom hole pressure (BHP) \\
\# Measurement times/well & 3 & 2 \\
$\tau$ & 1.1 & 1.1 \\
$\eta^{2} \tau^{2}$ & $1.45 \mathrm{e} 1$ & $2.18 \mathrm{e} 1$ \\
$\sigma$ & $1 \mathrm{e}-2$ & $1.5[\mathrm{psi}]$ \\
\hline
\end{tabular}

and (D)) matched almost exactly not only the measurements but also the entire interval of waterflood. In Table 6.4 we display the convergence history. As we experienced in Chapter 4 , for a SNR smaller we obtain faster convergence since we require less accuracy in the inverse model prediction.

TABLE 6.4: Convergence Results. Experiment VI.

\begin{tabular}{lll}
\hline & Experiment VI(a) & Experiment VI(b) \\
\hline (iteration) $n$ & $\left\|d_{p}^{\eta}-\mathcal{L}\left(f_{w, F}^{n}\right)\right\|_{C^{-1}}^{2}$ & $\left\|d_{p}^{\eta}-\mathcal{L}\left(f_{w, F}^{n}\right)\right\|_{C^{-1}}^{2}$ \\
\hline 1 & $3.576 \mathrm{e} 2$ & $1.509 \mathrm{e} 5$ \\
2 & $2.958 \mathrm{e} 1$ & $1.116 \mathrm{e} 4$ \\
3 & $7.956 \mathrm{e} 0$ & $8.766 \mathrm{e} 3$ \\
4 & - & $4.345 \mathrm{e} 2$ \\
5 & - & $1.552 \mathrm{e} 0$ \\
\hline
\end{tabular}

\subsection{A Sequential Implementation}

In Section (6.3) we presented a formulation for the identification of petrophysical properties given measurements which were all collected during the time interval of interest. However, in practical applications it is also relevant to consider a sequential approach of the history matching problem. For this reason we now describe an ad-hoc implementation of the IRBS for the sequential estimation of absolute permeability given dynamic data. In the IRBS, the interval of time is given by the forward model. Therefore, we formulate a sequence of identification problems for which the initial guess of the current interval is the estimated parameter from the previous one. The procedure is summarize in the following

Algorithm 6 (Sequential IRBS). Given prior estimates $\left(Y, \phi_{r}, \theta_{\alpha}\right)$, define problem (6.31) in some initial interval of time $\left[t_{0}, t_{1}\right]$ with an initial condition $\left[p, s, p_{b h}^{l}, q^{l}\right]_{t_{0}}$. 


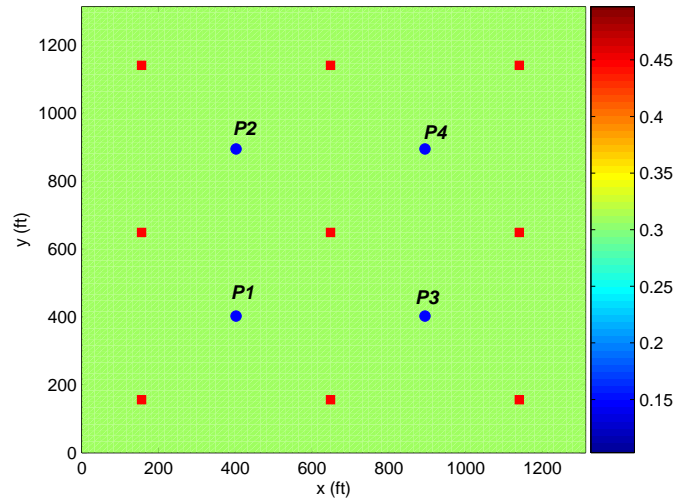

(A) Well Configuration

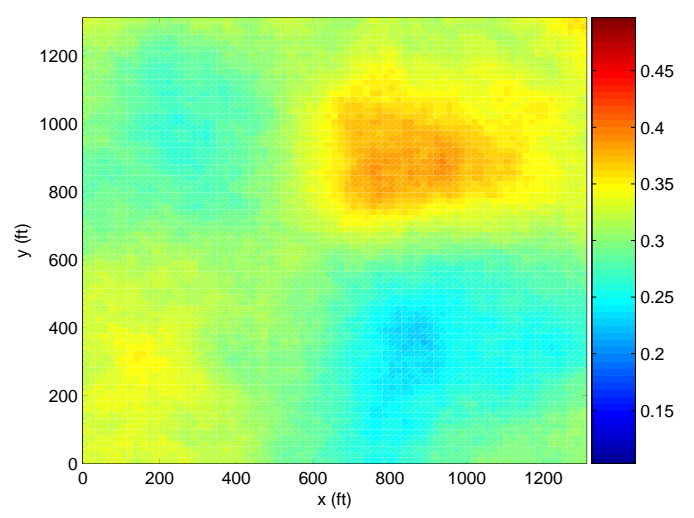

(c) IRBS-KL (Exp VI(a))

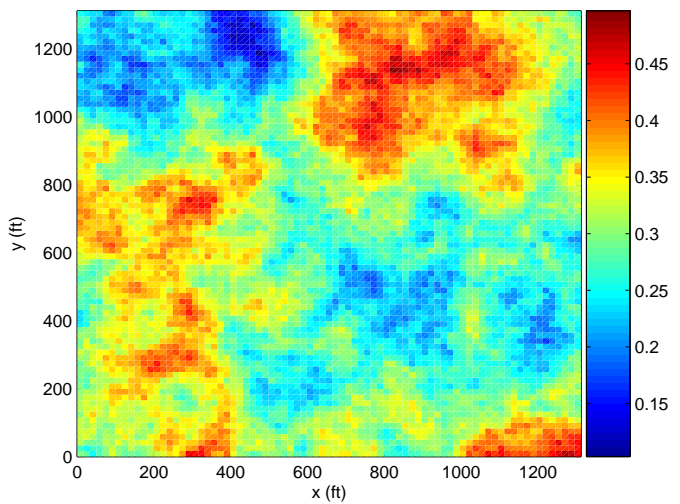

(B) True

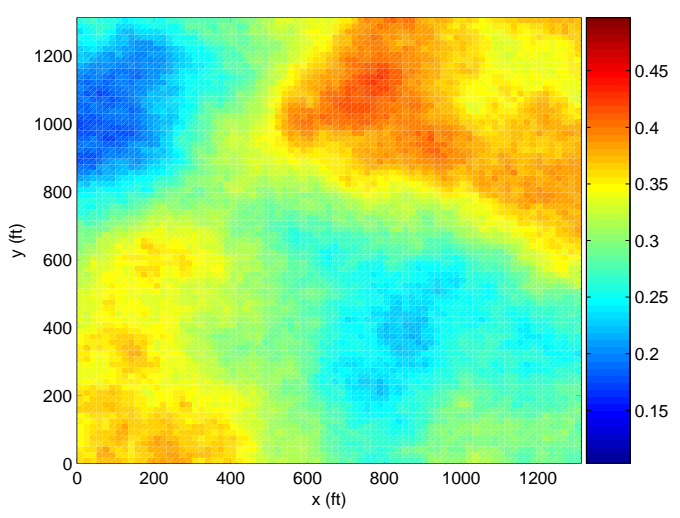

(D) $\operatorname{IRBS-KL}(\operatorname{Exp} V I(b))$

Figure 6.4: Experiment V(b). Porosity fields. 


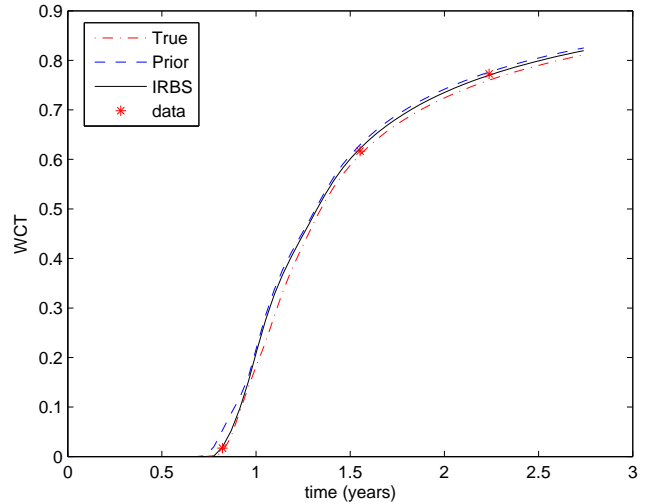

(A) Well P1 Exp VI(a)

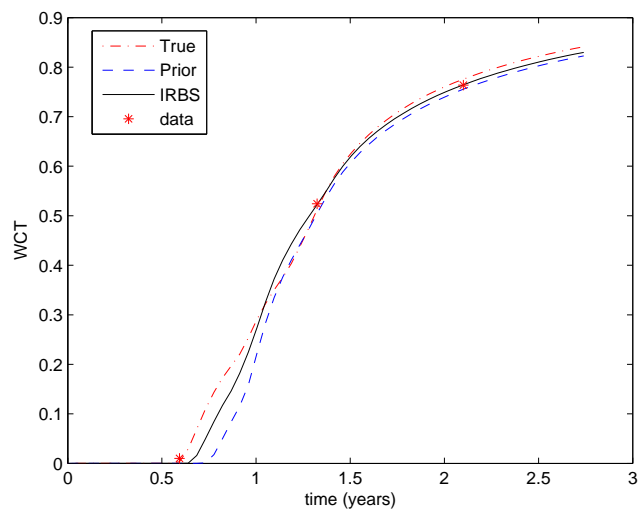

(c) Well P2 Exp VI(a)

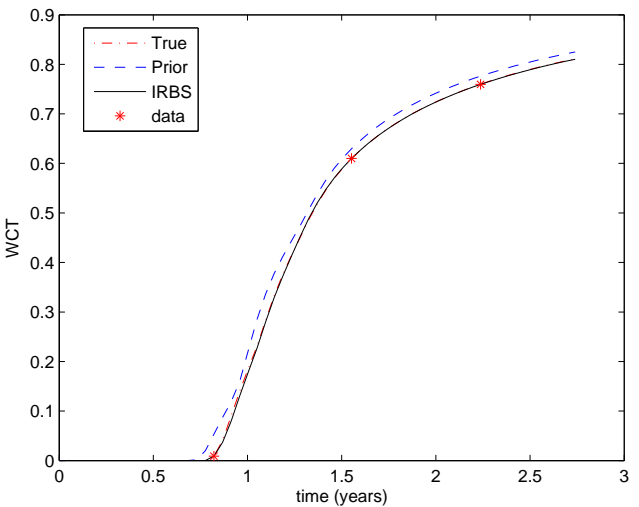

(B) Well P1 Exp VI(b)

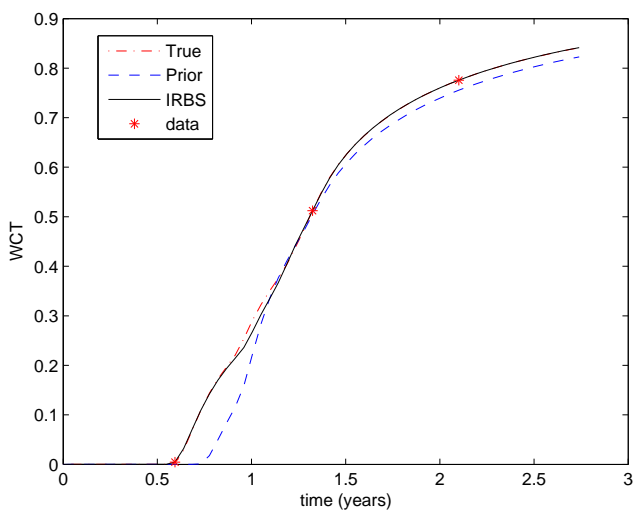

(D) Well P2 Exp VI(b)

FiguRE 6.5: Experiment VI(a)-(b). Water cut (WCT) history matching. Wells P1-P2. 


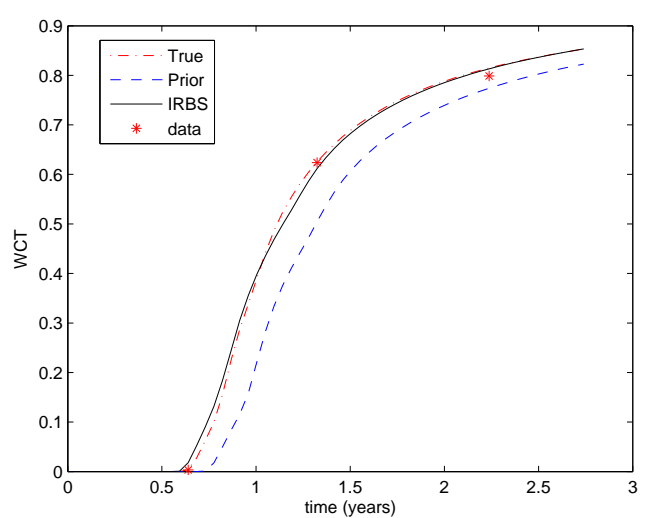

(A) Well P3 Exp VI(a)

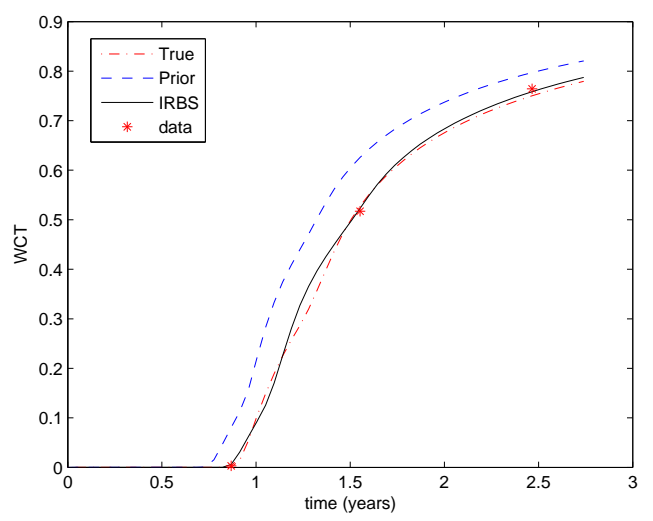

(c) Well P4 Exp VI(a)

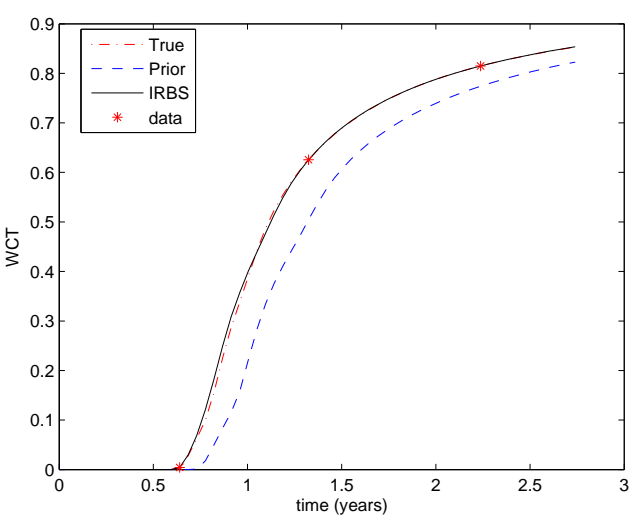

(B) Well P3 Exp VI(b)

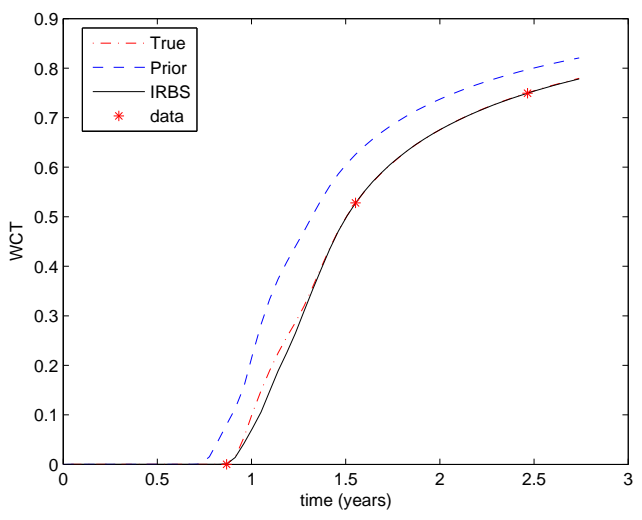

(D) Well P4 Exp VI(b)

FiguRE 6.6: Experiment VI(a)-(b). Water cut (WCT) history matching. Wells P3-P4. 
(1) Apply IRBS (Algorithm \& with Step (3) given by Algorithm 5) for measurements collected at $\left[t_{n-1}, t_{n}\right]$, to obtain, after convergence, estimate $\left(Y, \phi_{r}, \theta_{\alpha}\right)$

(2) With the new parameters $\left(Y, \phi_{r}, \theta_{\alpha}\right)$, compute the forward model solution $\left(p, s, p_{b h}^{l}, q^{l}\right)$ in interval $\left[t_{n-1}, t_{n}\right]$.

(3) Set $\left[p, s, p_{b h}^{l}, q^{l}\right]_{t_{n-1}}=\left[p, s, p_{b h}^{l}, q^{l}\right]_{t_{n}}$.

We now validate this algorithm using a true permeability from the SPE model that we used in Chapter 4. In this case we select the first layer of the reservoir (Figure 6.7)(a). This field has been recently used in [38] for history matching with the EnKF and geological constraints parameterized with the discrete cosine transform. In [38] the original permeability was scaled to avoid issues in the forward model. In this experiment we use the same scaling $\left(K \in\left[10^{2} \mathrm{md}, 10^{5} \mathrm{md}\right]\right)$ and well-configuration of [38]. For simplicity we keep the injection rate (bottom hole pressure) constant at each injection (production) well. In order to apply the IRBS-KL we need a set of realizations consistent with the geology of the true permeability. Since this information is not provided in the SPE model, we compute the experimental semivariogram (see Figure 6.7 (b)) in two directions with azimuthal angles $\psi=0$ and $\psi=\pi / 2$. We observe from Figure 6.7 (b) that this field presents both zonal and geometrical anisotropy. Therefore, we use two structures to model the variogram of this field. In Figure 6.8 we show the experimental and the corresponding fitted model in the two directions, where the fitted model is given

$$
\gamma=0.35 \operatorname{Sph}_{90}(1860,300)+0.55 \operatorname{Sph}(333,333) .
$$

Then, 200 unconditional realization were generated consistent with (6.95). Some of those realizations are displayed Figure 6.9. Note that the structure of the "true permeability" was captured with model (6.95). We now use this set of realization to perform principal component analysis and obtain the KL parametrization to be use in the IRBS. For the sequential IRBS we consider 4 assimilation intervals of equal length $\delta T=0.45 y e a r s$. Each assimilation time is taken at the middle of the time window. Some assimilation information is displayed in Table 6.5. The following results include a total of 44 months of history matching followed by 22 months of prediction. In Figure 6.10 we present the log-permeability estimates obtained for the assimilated intervals and in Table 6.6 we show convergence performance. History matching is presented in Figures 6.11 and 6.12.

Note that after the first assimilation time $\left(0, t_{1}\right)$, the IRBS-KL produces a permeability with a good visual agreement to the true log-permeability. In fact, for the subsequent intervals the estimate of the log-permeability does not exhibits substantial change. This phenomena is reflected in Table 6.6. After the first assimilation time, convergence is achieved 


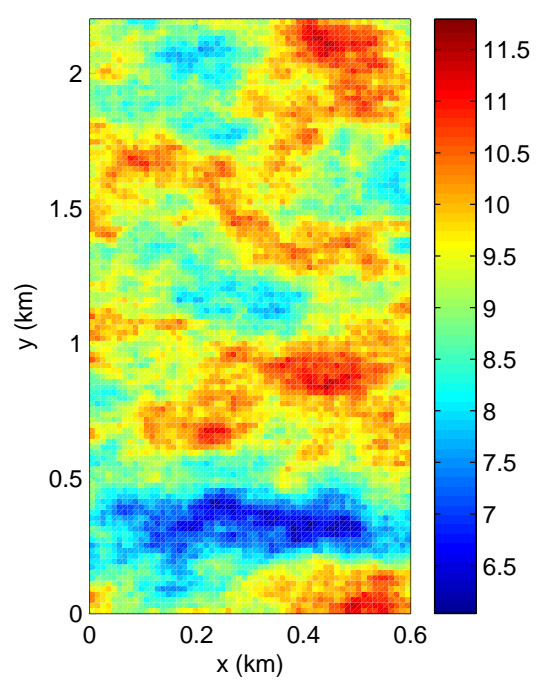

(A) SPE Permeability

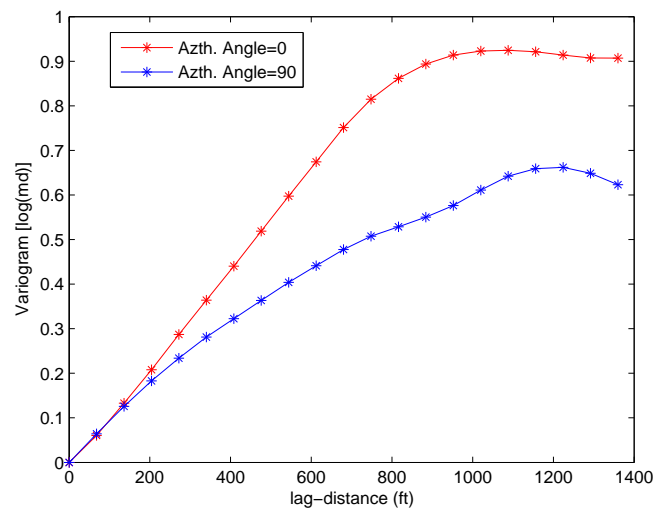

(B) Experimental Semivariograms

FIgURE 6.7: Experiment VI. SPE log-permeability and experimental semivariograms.

TABLE 6.5: Information for data assimilation. Experiment $V$.

\begin{tabular}{lll}
\hline Well type & Production & Injection \\
\hline Measurement type & total flow rate & Bottom hole pressure $(\mathrm{BHP})$ \\
$\tau$ & 1.1 & 1.1 \\
$\eta^{2} \tau^{2}$ & $9.68 \mathrm{e} 0$ & $1.82 \mathrm{e} 1$ \\
$\sigma$ & $10[\mathrm{bbl} / \mathrm{day}]$ & $1.5[\mathrm{psi}]$ \\
\hline
\end{tabular}

in the first iteration. On the other hand, from Figures 6.11 and 6.12 we see that after the first assimilation time, those subtle changes in log-permeability are indeed contributing to the matching of measurements.

\subsubsection{IRBS Vs EnKF}

One of our future goals is to incorporate the IRBS within the close-loop approach for reservoir management. The results of the previous section were encouraging since the IRBS is capable of producing updates of the petrophysical properties consistent to dynamics data and geological constraints. We now compare the results obtained in the previous section with those obtained with the implementation of EnKF.

To our best knowledge the EnKF has not been applied to the estimation of absolute 


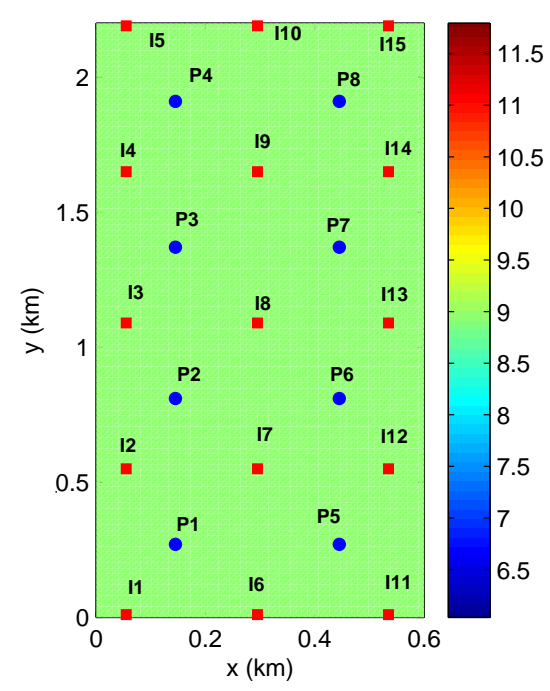

(A) SPE Permeability

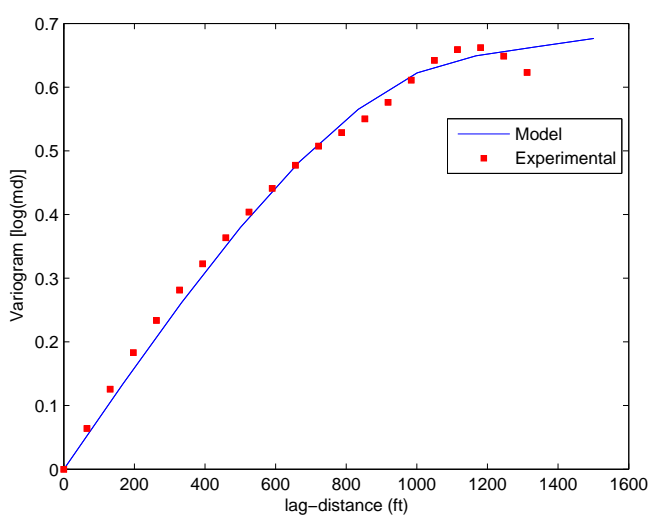

(в) $\psi=0$

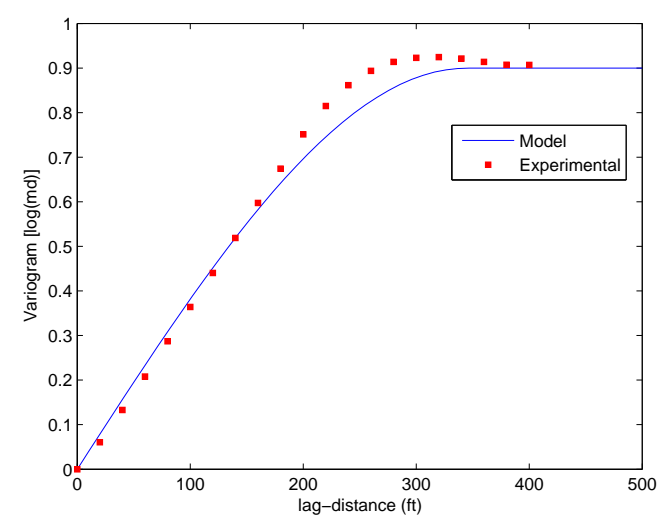

(C) $\psi=\pi / 2$

FiguRE 6.8: Experiment VI. Initial log-permeability and variograms. 


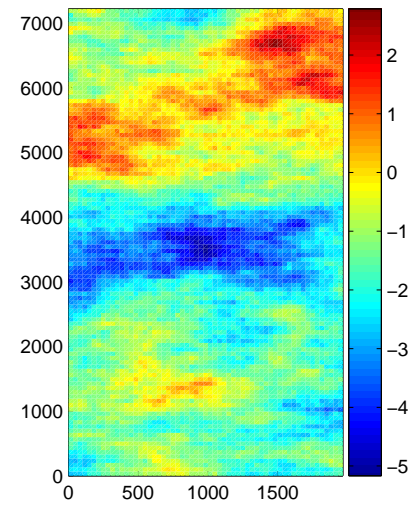

(A) Realization 1

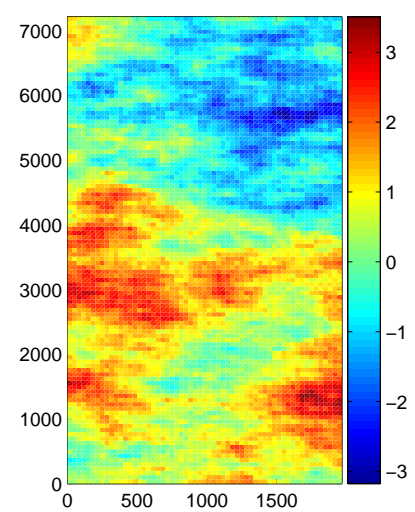

(D) Realization 4

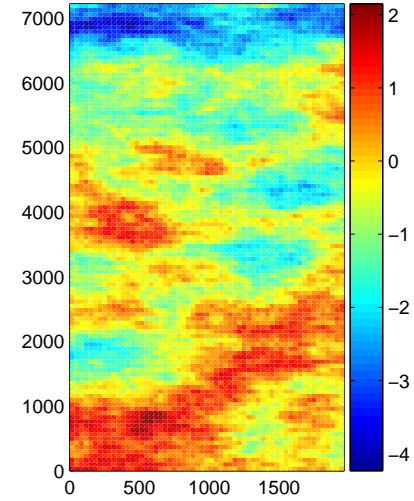

(B) Realization 2

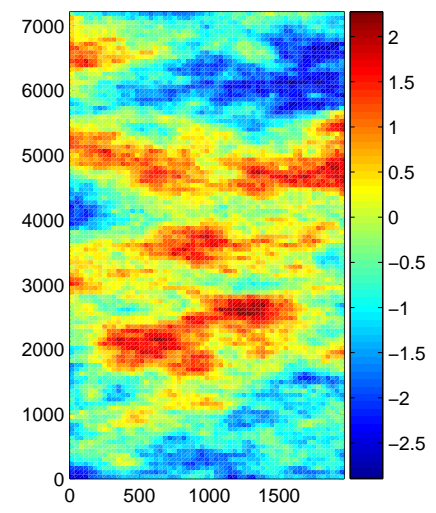

(E) Realization 5

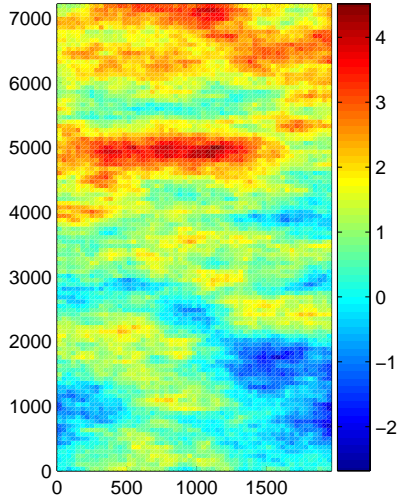

(C) Realization 3

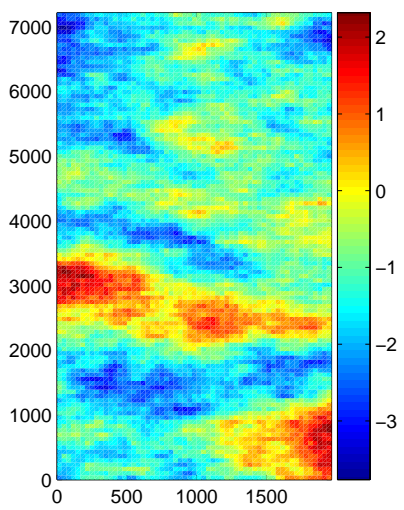

(F) Realization 6

FiguRE 6.9: Experiment VI. Stochastic realizations for Experiment VII. 


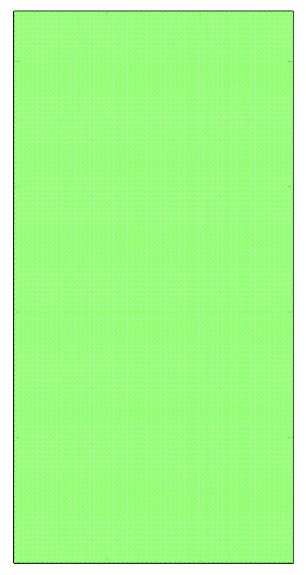

(A) Prior

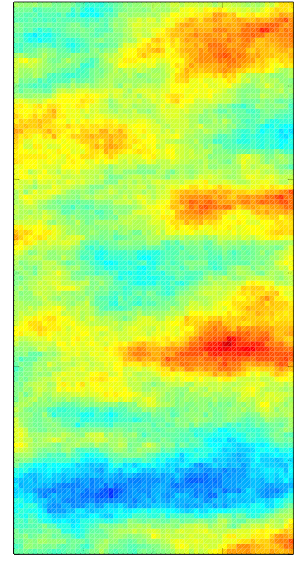

(D) $\left(t_{2}, t_{3}\right)$

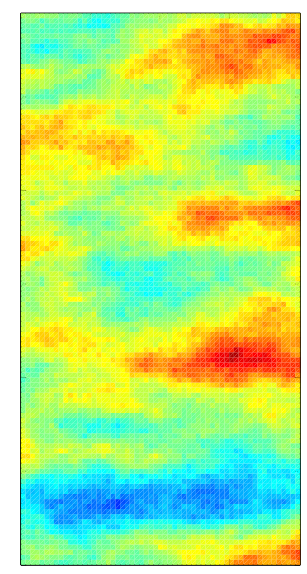

(B) $\left(0, t_{1}\right)$

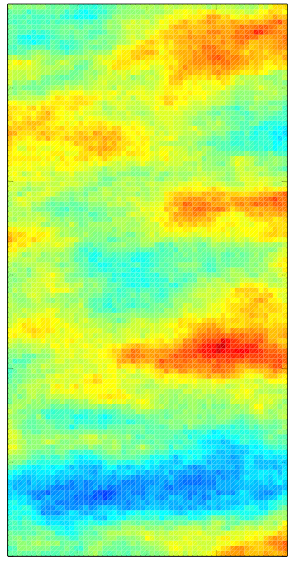

(E) $\left(t_{3}, t_{4}\right)$

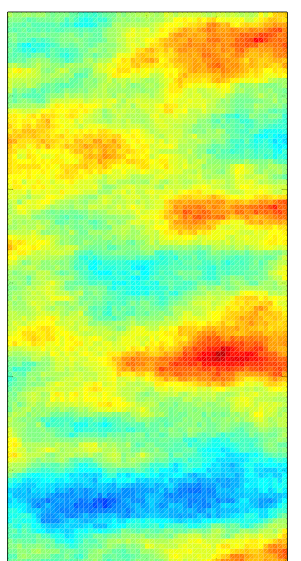

(C) $\left(t_{1}, t_{2}\right)$

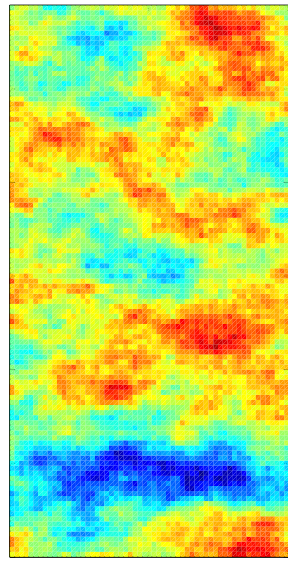

(F) True

Figure 6.10: Experiment VI. Log permeability fields [n md] computed with sequential IRBS-KL. 


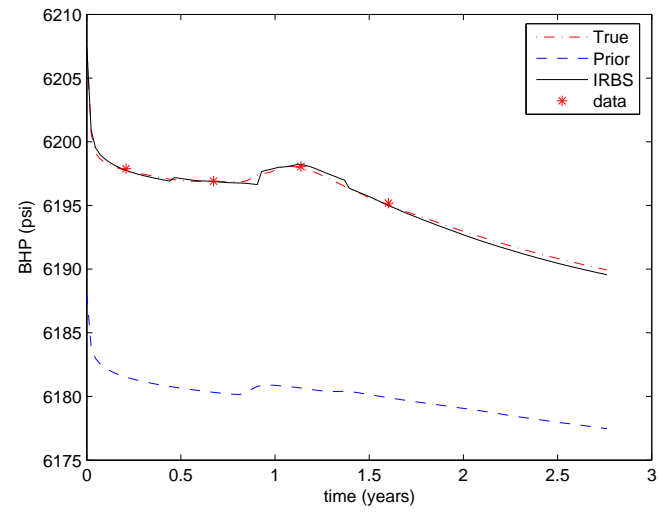

(A) $\mathrm{PHB}($ Well I1)

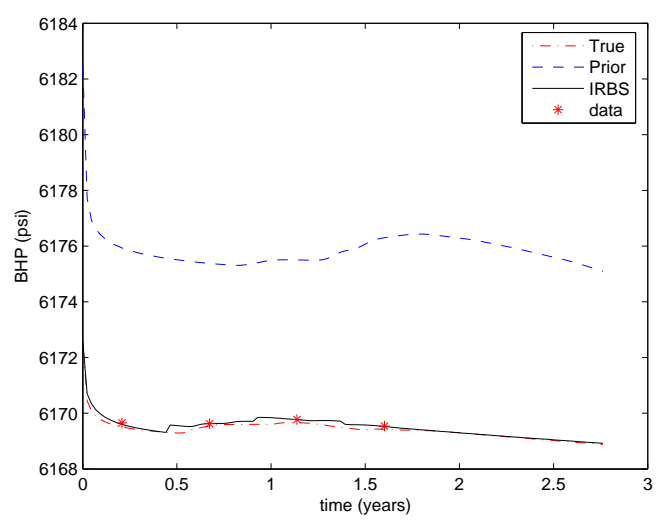

(c) PHB (Well I13)

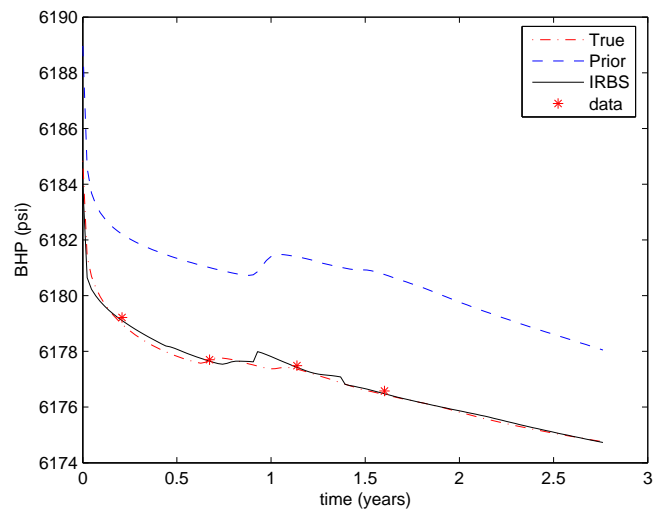

(E) $P H B($ Well I5)

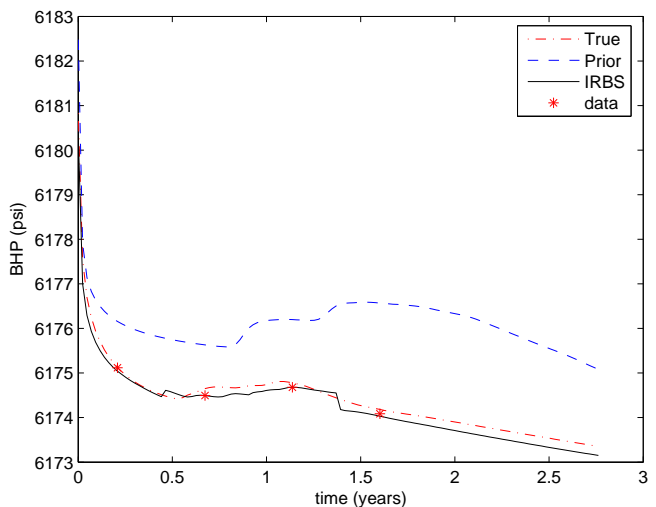

(в) $\mathrm{PHB}($ Well Ir)

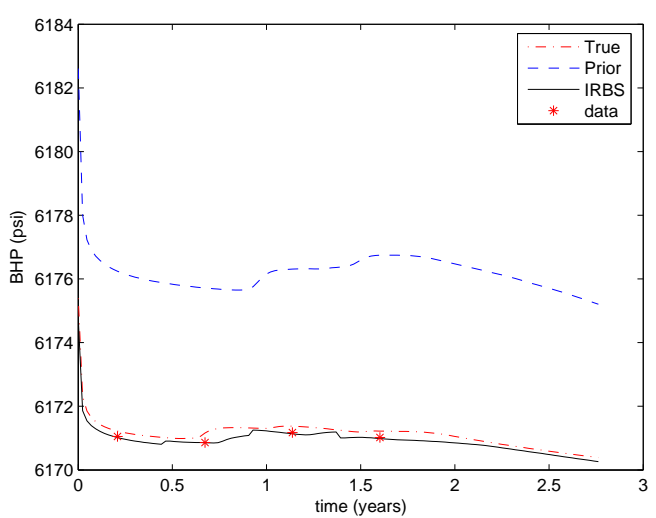

(D) $\mathrm{PHB}($ Well I9)

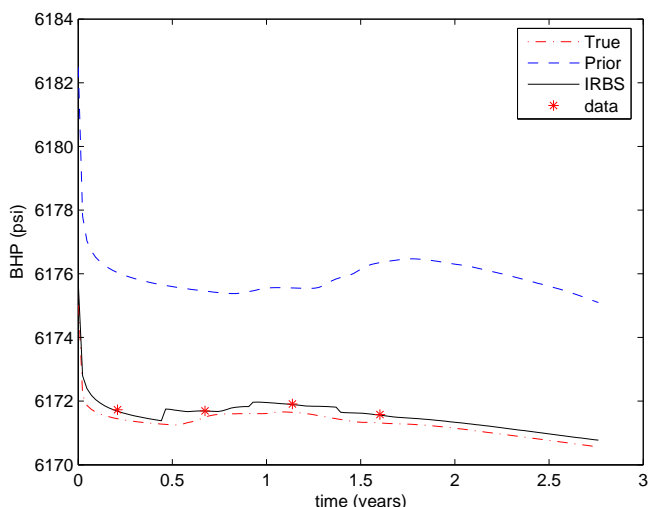

(F) $\mathrm{PHB}($ Well I3)

Figure 6.11: Experiment $\operatorname{VII}(a)$. BHP history matching. 


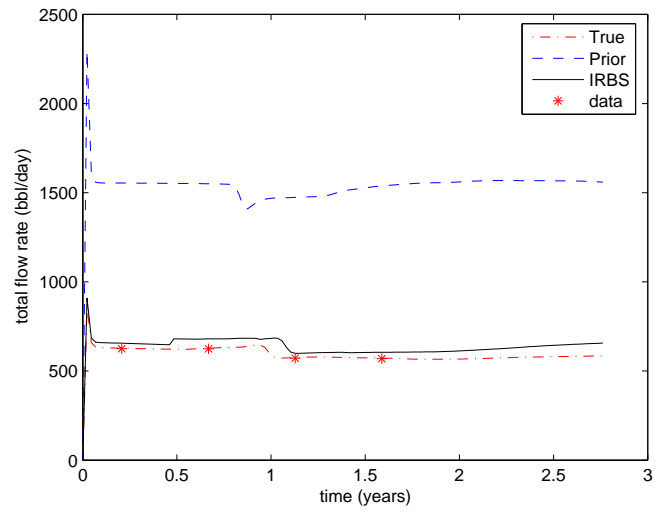

(A) flow rate (Well P1)

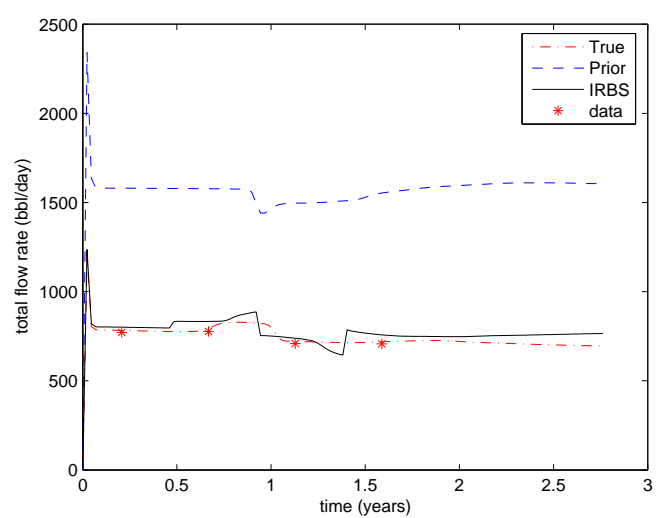

(c) flow rate (Well P4)

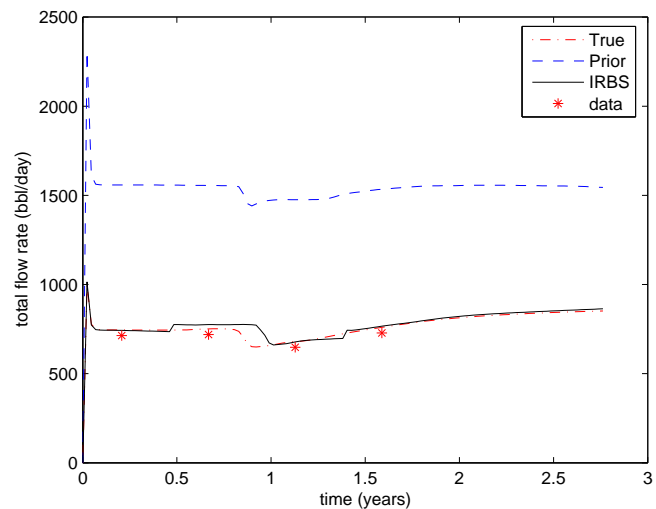

(E) flow rate (Well Pr)

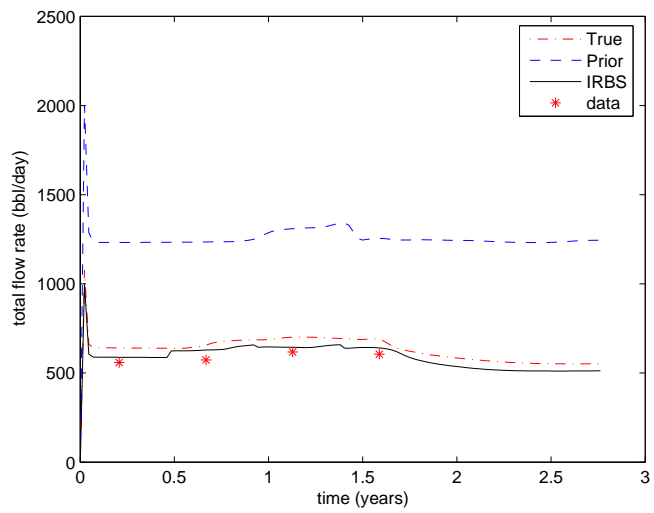

(B) flow rate (Well P3)

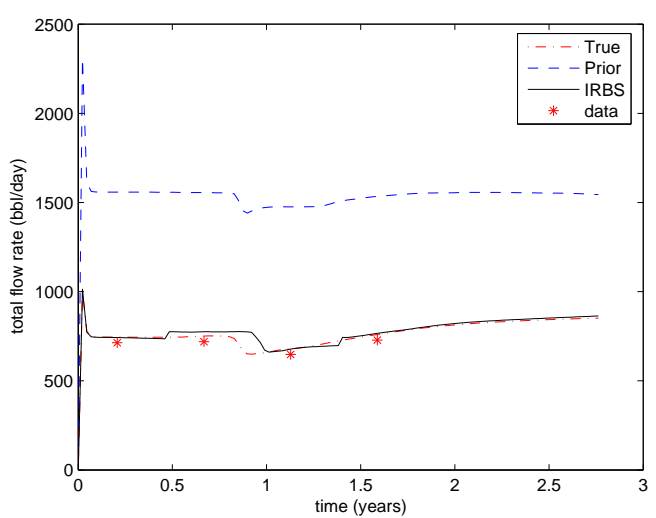

(D) flow rate (Well P5)

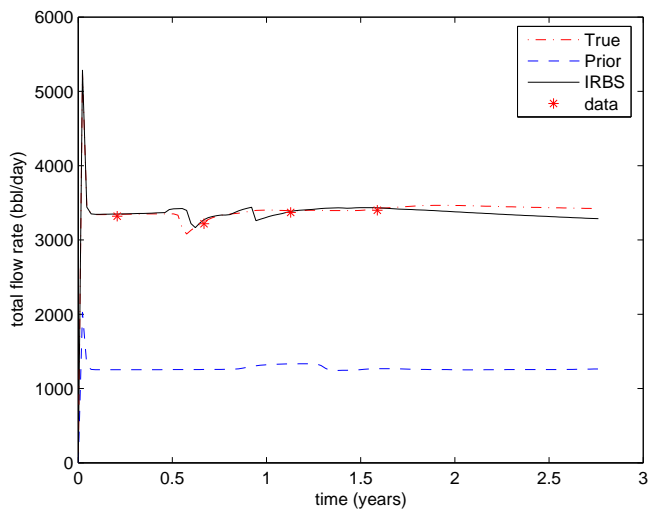

(F) flow rate (Well P8)

FiguRE 6.12: Experiment VII(a). Total flow rate history matching. 
TABLE 6.6: Convergence Results. Experiment VII(a).

\begin{tabular}{lll}
\hline $\begin{array}{l}\text { Assimilation Interval: } \\
\text { (iteration) } n\end{array}$ & {$[0,0.445]($ years $)$} & $\left\|d_{I}^{\eta}-\mathcal{L}\left(q_{F}^{n}\right)\right\|_{C^{-1}}^{2}$ \\
\hline 1 & $4.17445 \mathrm{e} 4$ & $5.5885 \mathrm{e} 3$ \\
2 & $3.2877 \mathrm{e} 4$ & $1.678 \mathrm{e} 2$ \\
3 & $1.0709 \mathrm{e} 4$ & $1.03 \mathrm{e} 1$ \\
4 & $9.9 \mathrm{e} 2$ & $5.3104 \mathrm{e} 0$ \\
\hline Assimilation Interval: & {$[0.445,0.913]($ years $)$} & \\
(iteration) $n$ & $\left\|d_{I}^{\eta}-\mathcal{L}\left(q_{F}^{n}\right)\right\|_{C^{-1}}^{2}$ & $\left\|d^{\eta}-\mathcal{L}\left(p_{b h}\right)\right\|_{C^{-1}}^{2}$ \\
\hline 1 & $4.1484 \mathrm{e} 2$ & $1.42 \mathrm{e} 2$ \\
2 & $6.603 \mathrm{e}-1$ & 4.086 \\
\hline Assimilation Interval: & {$[0.913,1.3813]($ years $)$} & \\
(iteration) $n$ & $\left\|d_{I}^{\eta}-\mathcal{L}\left(q_{F}^{n}\right)\right\|_{C^{-1}}^{2}$ & $\left\|d^{\eta}-\mathcal{L}\left(p_{b h}\right)\right\|_{C^{-1}}^{2}$ \\
\hline 1 & $2.2475 \mathrm{e} 3$ & $4.27 \mathrm{e} 2$ \\
2 & 6.2354 & 1.2673 \\
\hline Assimilation Interval: & {$[1.3813,1.8495]($ years $)$} & \\
$($ iteration $) n$ & $\left\|d_{I}^{\eta}-\mathcal{L}\left(q_{F}^{n}\right)\right\|_{C^{-1}}^{2}$ & $\left\|d^{\eta}-\mathcal{L}\left(p_{b h}\right)\right\|_{C^{-1}}^{2}$ \\
\hline 1 & $6.0 \mathrm{e} 1$ & $2.67 \mathrm{e} 1$ \\
2 & $6.4 \mathrm{e}-1$ & $7.65 \mathrm{e} 0$ \\
\hline
\end{tabular}

permeability with a KL parametrization. Therefore, we believe this is a good opportunity to take that approach. For technical details of the general implementation of the EnKF we refer the reader to [22].

The incorporation of the KL expansions to the EnKF is trivial. Instead of using the standard formula for updating the full vector of grid-block log-permeability, we rather update variable $\xi$ in expression (5.10). The filtering problem is therefore posed on the parameter of the linear geological constraints. We consider an initial ensemble of parameters $(\xi)$ with 120 members. The ensemble size was selected to obtain a final cputime of $2 e 3 \mathrm{sec}$ which is the same execution time of the IRBS-KL implementation of the previous section. Note that the initial ensemble produces 120 log-permeability realizations consistent with (6.95). The assimilation times in the EnKF implementations are the same as those for the IRBS. However, note that in the EnKF the implementation is fully sequential. In Figure 6.13 we present estimates of log-permeability at each assimilation time. Each estimate was obtained from expression (5.10), by using the updated ensemble mean $\xi_{\text {mean }}$ at the assimilation time. The history matching plots at some well locations are shown in Figure 6.14. Note that the spread of the ensemble is reduced as more measurements are assimilated. 


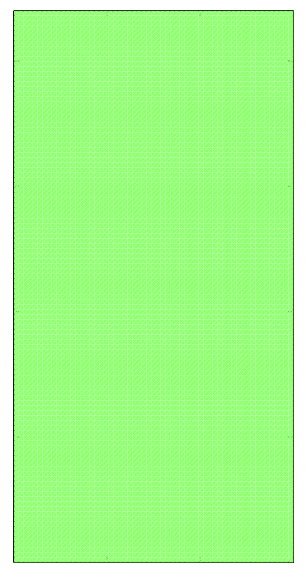

(A) Prior

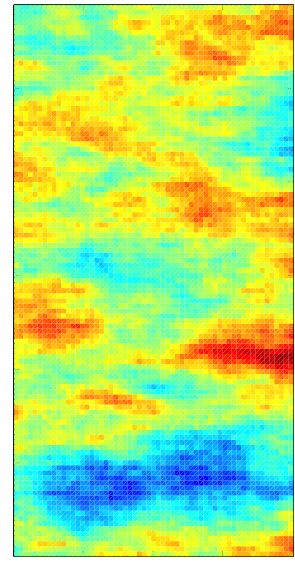

(D) $t=1.15$ years

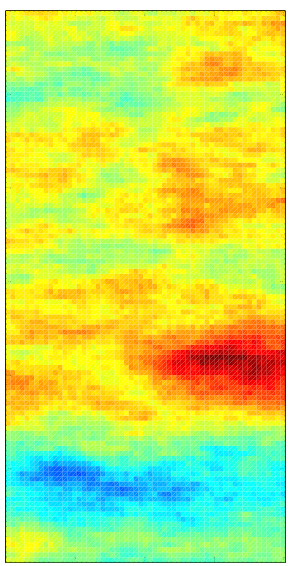

(в) $t=0.21$ years

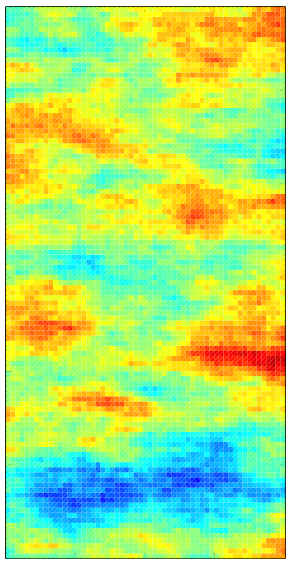

(E) $t=1.62$ years

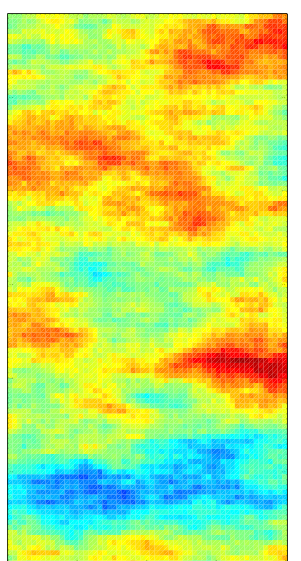

(c) $t=0.68$ years

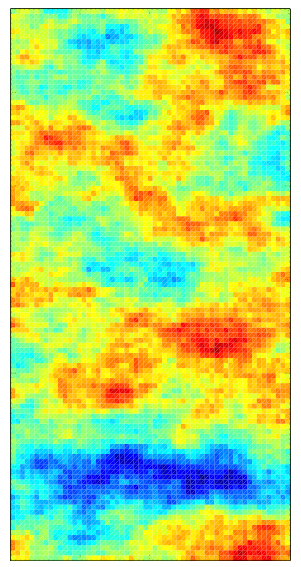

(F) True

FiguRE 6.13: Experiment VI(b).EnKF estimated log permeability fields [n md]. 


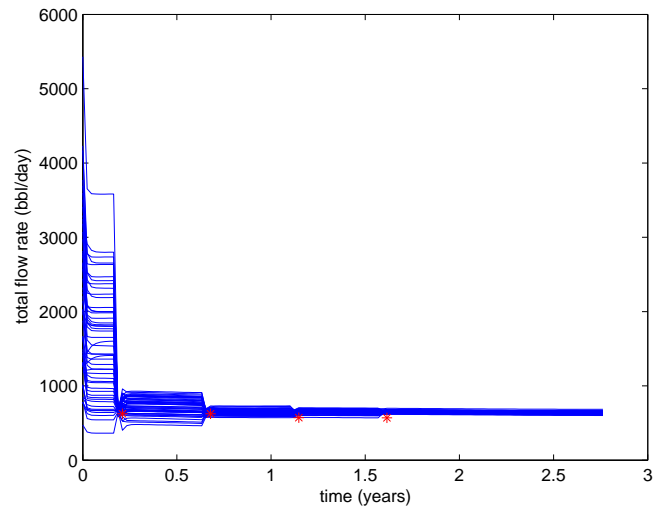

(A) Well P1 (flow rate)

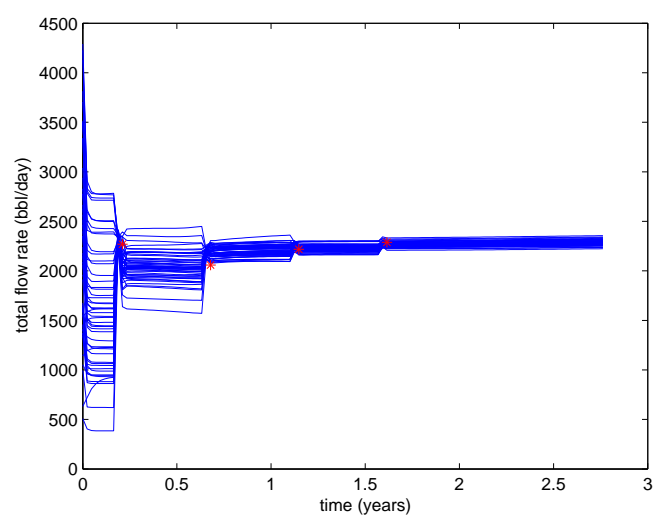

(c) Well P8 (flow rate)

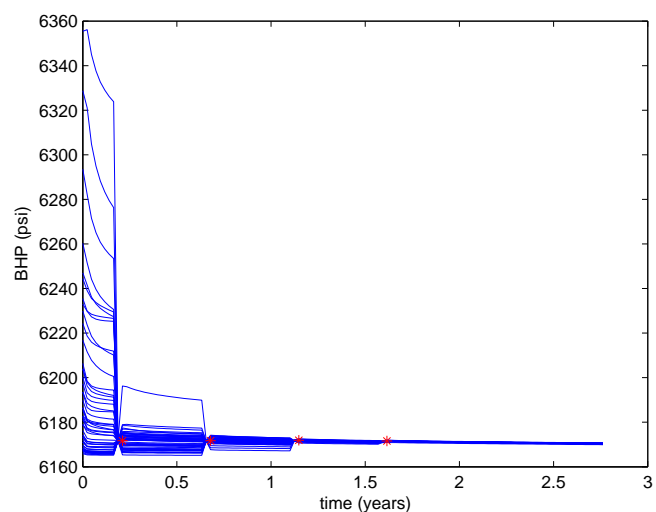

(E) Well I3 (BHP)

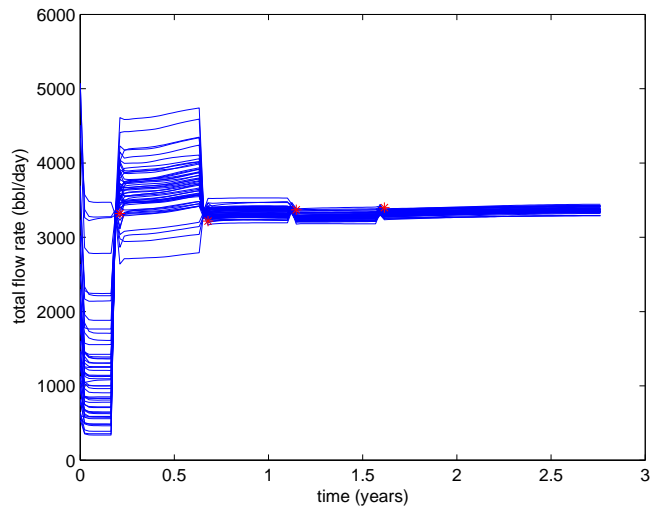

(B) Well P6 (flow rate)

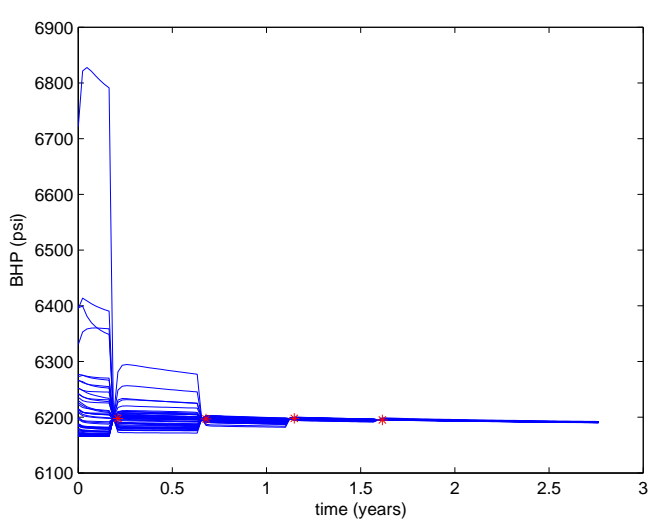

(D) Well I1 (BHP)

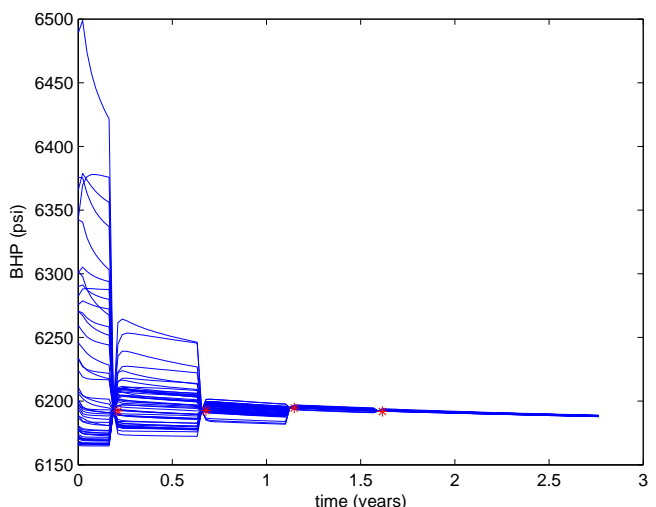

(F) Well I11 (BHP)

FiguRE 6.14: Experiment VI(b). EnKF history matching. 
In order to compare the results with those obtained with IRBS-KL we now present in Figures $6.15,6.16$ the history matching curves at some wells computed with both IRBS-KL and the EnKF mean. Although the EnKF mean produces good fit to the measurements, clearly the IRBS is more accurate especially at the initial assimilation intervals. Therefore, if we needed to predict a more accurate reservoir performance, the IRBS would be a better option for the estimation of log-permeability. However, when we compare Figure 6.13 with Figure 6.10, we find that at the third assimilation time, the EnKF has generated more consistent estimate of the permeability than the IRBS. This phenomena clearly reflects the fact that in the EnKF, the covariance of the ensemble is updated at each assimilation time. In contrast, our implementation of the IRBS assumes a constant covariance during the whole experiment. In our previous results [36] we have found explicit formulas for updating covariance in terms of representers. Further investigation should address covariance update in the IRBS implementation. 


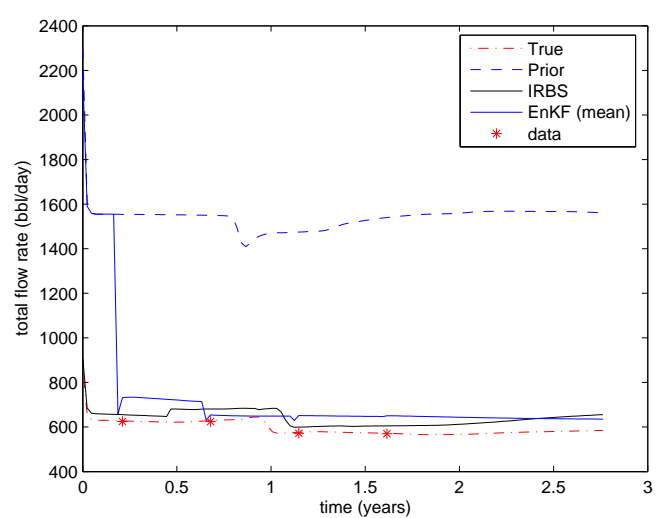

(A) Well P1 (flow rate)

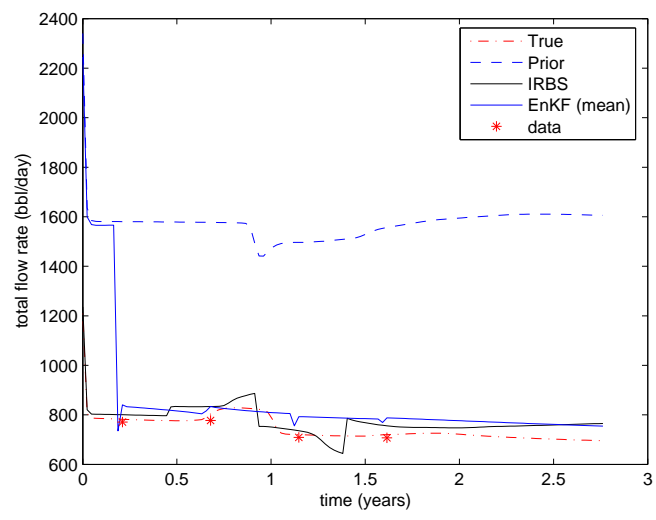

(c) Well P4 (flow rate)

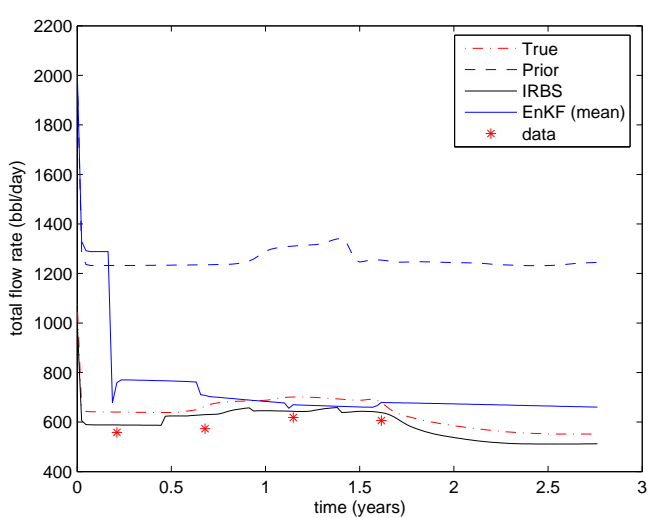

(в) Well P3 (flow rate)

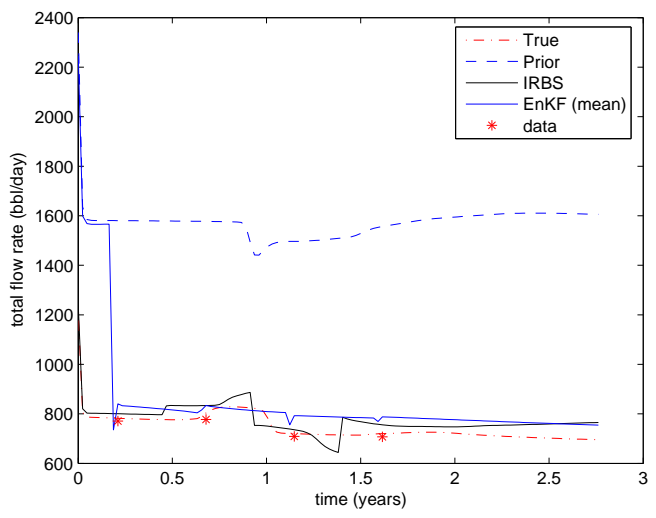

(D) Well P8 (flow rate)

Figure 6.15: EnKF History-Matching (total flow rate). 


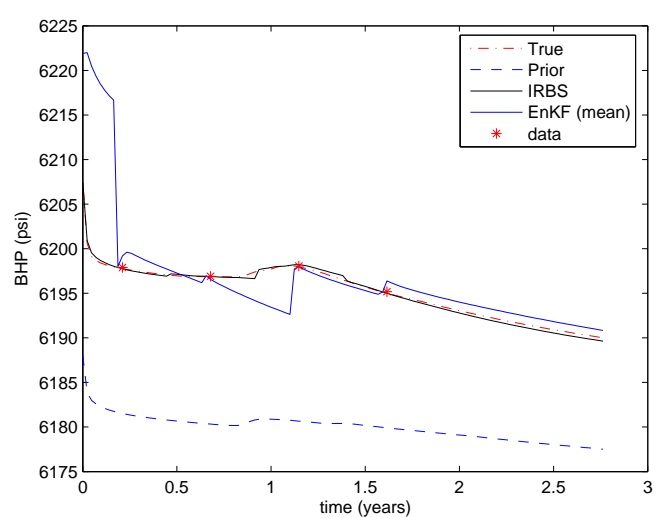

(A) Well I1 (BHP)

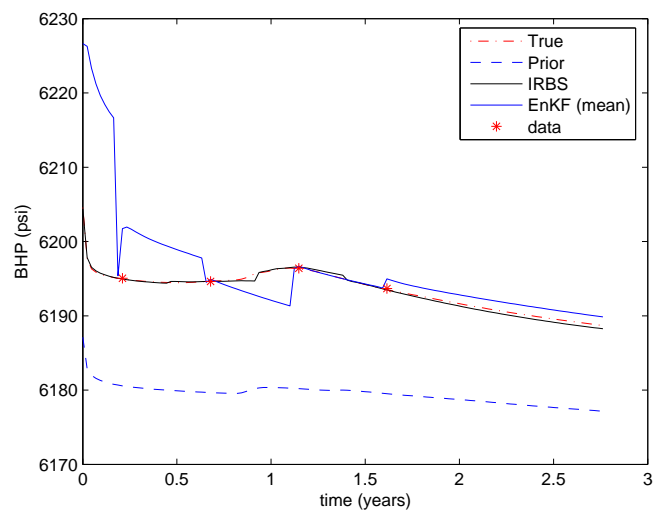

(c) Well I6 (BHP)

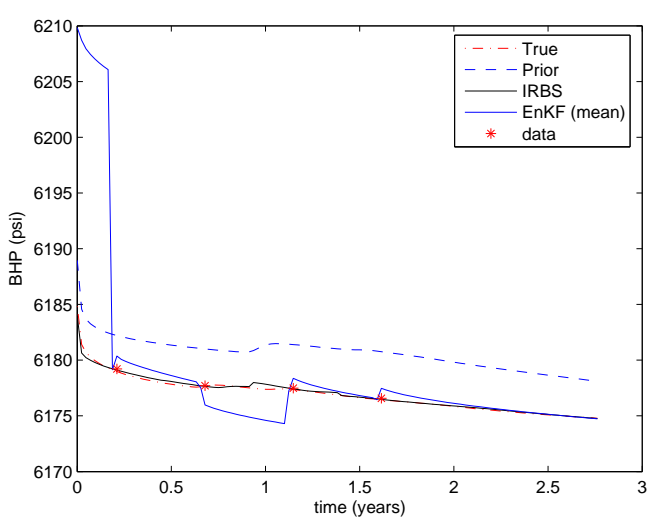

(в) Well I5 (BHP)

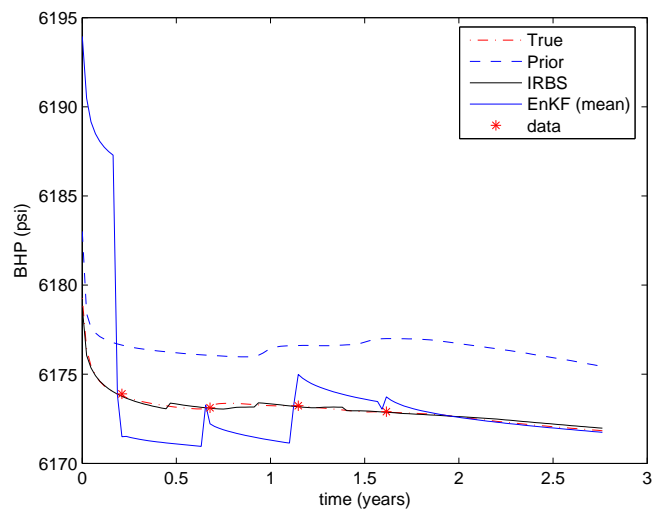

(D) Well I13 (BHP)

FiguRE 6.16: EnKF History-Matching (bottom hole pressure). 


\section{Chapter 7}

\section{Conclusions and Future Research}

In this dissertation we presented the theory and application of an iterative representerbased scheme (IRBS) for the identification of petrophysical properties in reservoir models. The objective of the proposed IRBS was to generate improved estimates of the reservoir properties that honor dynamic data collected at wells. The inverse problem was defined within an abstract framework of parameter identification. Standard techniques allowed us to establish general conditions for convergence of the IRBS. The abstract framework is very convenient when the forward model is described by a PDE such as the linear model studied in Chapter 4. By means of energy inequalities in Sobolev spaces, we proved local convergence of the IRBS applied to the identification of the coefficient of the second order elliptic operator in a parabolic linear forward model. As in any Gauss-Newton method, we found that convergence of the IRBS is insured when the initial guess is close enough to a solution of the parameter identification problem. Our numerical results show that even when the initial guess is far from the "truth", fast convergence is achieved. Moreover, the identified parameter always showed substantial improvement with respect to the initial guess.

One aspect that distinguishes the IRBS from other quasi-Newton methods is the implementation of the representer method for solving the linearized inverse problem on each iteration. Although there are other linear programming methods that can be used to solve the linearized problem, the representer method provides an efficient implementation that exploits two specific properties of the inverse problem: the penalty functional is quadratic and the number of measurements is finite. These properties are the essence of the computational efficiency of the representer method which became evident when we compared the IRBS with a direct implementation of a standard gradient-based history matching technique. In addition, the implementation of the IRBS is general and robust whereas the standard 
gradient-based techniques require scaling and damping which are mostly determined on a case-by-case basis [28].

As we indicated earlier, the mathematical framework of the IRBS was inspired by the theory of Tikhonov regularization for parameter identification. In contrast to the classical parameter identification approach [32], our approach incorporated prior knowledge via the prior error covariance of the uncertain parameter. Our numerical results showed that even with poor prior knowledge, the reconstructions captured the main heterogeneities of the fields. Furthermore, when linear geological constrains were imposed, the dimension of the parameter space was reduced. As a consequence, the ill-posedness of the inverse problem was partially alleviated, and the IRBS-KL produced better and geologically consistent estimates of the petrophysical properties of the reservoir.

The IRBS-KL was applied to the estimation of petrophysical properties in an oilwater reservoir. We presented a formal derivation of the IRBS for the estimation of porosity, permeability and relative permeability curves. In this derivation, the linearized well model was the measurement functional of the linearized inverse problem at each iteration. Although we used a simple well model, encouraging results were obtained. However, we observed that more realistic well models should be used for a better identification near the wellbore. Moreover, a deep understanding of the relation parameter-observation is required to establish the appropriate measurements during the assimilation and avoid redundancy in the inversion.

The formulation of the IRBS for the estimation of reservoir properties was not only efficient and robust but also convenient for the closed-loop approach. We derived an ad hoc sequential implementation of the IRBS and compared its performance with the ensemble Kalman filter. It was found that the IRBS provided better estimates at the beginning of the assimilation window. The numerical results were promising and indicated that the IRBS may become a tool for parameter estimation in reservoir modeling. With the integration of seismic data and smart-wells technology, better prior knowledge and reliable measurements may result in an improved reservoir characterization.

The theory and application presented in this dissertation may be extended in many ways. With respect to the theory, one of the following steps is to derive the necessary conditions for convergence of the IRBS applied to the forward model of Chapter 6 . The main challenge of this research is the proper application of existence and uniqueness results of the forward model $[2,72]$. This theoretical analysis may be helpful to understand the capabilities and limitations of the IRBS. Additionally, one the most important aspects of the inverse problem is to determine the identifiability of the petrophysical properties of the reservoir. Where and when should we measure the state? Should we expect local identifiability of the 
uncertain property? Some of those concerns have already been addressed for the simplified model of Buckley-Leverett in one dimension. However, for the two-phase model utilized in the present work, those are still open questions whose answer may be the parameterobservation relation so valuable for improving the inverse estimations.

With respect to the application, the next step is the analysis of the well-model in the inverse estimation. A natural approach would be the implementation of the IRBS with a more accurate well-model. A different approach is the incorporation of the uncertainty in the well model as part of the inverse problem. This could be accomplished following our previous work on state estimation [36] by adding the well-model error as a forcing term in the model equations. Furthermore, in order to identify realistic geological models, it is essential to incorporate new parameterizations that preserves multipoint statistics. The natural approach is to implement the IRBS coupled with the nonlinear PCA used in [59]. Finally, it is important to develop a version of the IRBS for the problem of uncertainty quantification. The covariance must be updated at some point of the implementation of the IRBS. In particular, in the sequential IRBS-KL, the parametrization should be updated after each assimilation interval of time so that the geological information can be fully captured. 


\section{Appendix A}

\section{Proofs of Chapter 3}

In this section we provide rigorous proofs of Lemma 2 and Theorems 2 and 3 of Chapter 3. We first present a technical lemma concerned with the monotonicity of the iterates. This lemma is analogous to Proposition 2.1 of [32]. However, the main difference to the work of [32] is that the nonlinearity condition (Assumption 2) is imposed to the implicit nonlinear forward operator $G(k, s)=0$.

\section{A.1 Proof of Lemma 2}

Since (A1) and (F1)-(F3) hold, the assumptions on Theorem 1 are satisfied. Then, $k^{n}$ is well defined in terms of (3.21). We recall that $R^{n}$ defined by (3.16) is positive semidefinite. Since $C$ is by hypothesis positive definite, then $R^{n}+C$ is symmetric positive definite. Note now that

$$
\begin{gathered}
\left\|k_{*}-k^{n}\right\|_{\mathcal{K}}^{2}=-\left\|k^{n}-k^{n-1}\right\|_{\mathcal{K}}^{2}+2\left\|k^{n}-k^{n-1}\right\|_{\mathcal{K}}^{2} \\
-2\left\langle k_{*}-k^{n-1}, k^{n}-k^{n-1}\right\rangle_{\mathcal{K}}+\left\|k_{*}-k^{n-1}\right\|_{\mathcal{K}}^{2} .
\end{gathered}
$$

From expression (3.21) and using the linearity of the inner product it follows

$$
-2\left\langle k_{*}-k^{n-1}, k^{n}-k^{n-1}\right\rangle_{\mathcal{K}}=-2 \sum_{m j}^{M}\left[R^{n}+C\right]_{m j}^{-1}\left[d_{j}^{\eta}-\mathcal{L}_{j}\left(s_{F}^{n}\right)\right]\left\langle k_{*}-k^{n-1}, \gamma_{m}^{n}\right\rangle_{\mathcal{K}}
$$

From the definition of $\gamma_{m}^{n}$ (see (3.19)),

$$
\left\langle k_{*}-k^{n-1}, \gamma_{m}\right\rangle_{\mathcal{K}}=\mathcal{L}_{m}\left(s_{r}^{n}-s_{F}^{n}\right)
$$


where $s_{r}^{n}$ is the solution to

$$
D_{k} G\left(k^{n-1}, s_{F}^{n}\right)\left[k_{*}-k^{n-1}\right]+D G_{s}\left(k^{n-1}, s_{F}^{n}\right)\left[s_{r}^{n}-s_{F}^{n}\right]=0 .
$$

Substituting (A.3) into (A.2) and with some simple computations we find

$$
\begin{array}{r}
-2\left\langle k_{*}-k^{n-1}, k^{n}-k^{n-1}\right\rangle_{\mathcal{K}}=2 \sum_{m j}^{M}\left[R^{n}+C\right]_{m j}^{-1}\left[d_{j}^{\eta}-\mathcal{L}_{j}\left(s_{F}^{n}\right)\right]\left[d_{m}^{\eta}-\mathcal{L}_{m}\left(s_{r}^{n}\right)\right] \\
-2 \sum_{m j}^{M}\left[R^{n}+C\right]_{m j}^{-1}\left[d_{j}^{\eta}-\mathcal{L}_{j}\left(s_{F}^{n}\right)\right]\left[d_{m}^{\eta}-\mathcal{L}_{m}\left(s_{F}^{n}\right)\right] \\
=2\left[d^{\eta}-\mathcal{L}\left(s_{F}^{n}\right)\right]^{T}\left[R^{n}+C\right]^{-1}\left[d^{\eta}-\mathcal{L}\left(s_{r}^{n}\right)\right]-2\left[d^{\eta}-\mathcal{L}\left(s_{F}^{n}\right)\right]^{T}\left[R^{n}+C\right]^{-1}\left[d^{\eta}-\mathcal{L}\left(s_{F}^{n}\right)\right] .
\end{array}
$$

With similar arguments we obtain

$$
2\left\|k^{n}-k^{n-1}\right\|_{\mathcal{K}}^{2}=2\left[d^{\eta}-\mathcal{L}\left(s_{F}^{n}\right)\right]^{T}\left[R^{n}+C\right]^{-1} R^{n}\left[R^{n}+C\right]^{-1}\left[d^{\eta}-\mathcal{L}\left(s_{F}^{n}\right)\right]
$$

Then, the second and third terms of the right hand side of expression (A.1) can be written as

$$
\begin{array}{r}
2\left\|k^{n}-k^{n-1}\right\|_{\mathcal{K}}^{2}-2\left\langle k_{*}-k^{n-1}, k^{n}-k^{n-1}\right\rangle_{\mathcal{K}}=2\left[d^{\eta}-\mathcal{L}\left(s_{F}^{n}\right)\right]^{T}\left[R^{n}+C\right]^{-1}\left[d^{\eta}-\mathcal{L}\left(s_{r}^{n}\right)\right] \\
-2\left[d^{\eta}-\mathcal{L}\left(s_{F}^{n}\right)\right]^{T}\left[R^{n}+C\right]^{-1} C\left[R^{n}+C\right]^{-1}\left[d^{\eta}-\mathcal{L}\left(s_{F}^{n}\right)\right]
\end{array}
$$

where we have applied

$$
\left[R^{n}+C\right]^{-1}-\left[R^{n}+C\right]^{-1} R^{n}\left[R^{n}+C\right]^{-1}=\left[R^{n}+C\right]^{-1} C\left[R^{n}+C\right]^{-1} .
$$

We substitute (A.7) in (A.1) and use the fact that $\left\|k^{n}-k^{n-1}\right\|_{\mathcal{K}}^{2}>0$ to obtain

$$
\begin{aligned}
\left\|k_{*}-k^{n-1}\right\|_{\mathcal{K}}^{2}- & \left\|k_{*}-k^{n}\right\|_{\mathcal{K}}^{2}>-2\left[d^{\eta}-\mathcal{L}\left(s_{F}^{n}\right)\right]^{T}\left[R^{n}+C\right]^{-1}\left[d^{\eta}-\mathcal{L}\left(s_{r}^{n}\right)\right] \\
+ & 2\left[d^{\eta}-\mathcal{L}\left(s_{F}^{n}\right)\right]^{T}\left[R^{n}+C\right]^{-1} C\left[R^{n}+C\right]^{-1}\left[d^{\eta}-\mathcal{L}\left(s_{F}^{n}\right)\right] .
\end{aligned}
$$

Note that the second term in the right hand side of the previous expression satisfies

$$
\begin{array}{r}
2\left[d^{\eta}-\mathcal{L}\left(s_{F}^{n}\right)\right]^{T}\left[R^{n}+C\right]^{-1} C\left[R^{n}+C\right]^{-1}\left[d^{\eta}-\mathcal{L}\left(s_{F}^{n}\right)\right] \\
=2\left[d^{\eta}-\mathcal{L}\left(s_{F}^{n}\right)\right]^{T} C^{-1 / 2} C^{1 / 2}\left[R^{n}+C\right]^{-1} C^{1 / 2} C^{1 / 2}\left[R^{n}+C\right]^{-1} C^{1 / 2} C^{-1 / 2}\left[d^{\eta}-\mathcal{L}\left(s_{F}^{n}\right)\right] \\
=2\left[d^{\eta}-\mathcal{L}\left(s_{F}^{n}\right)\right]^{T} C^{-1 / 2} A^{2} C^{-1 / 2}\left[d^{\eta}-\mathcal{L}\left(s_{F}^{n}\right)\right],(
\end{array}
$$


where

$$
A \equiv C^{1 / 2}\left[R^{n}+C\right]^{-1} C^{1 / 2}
$$

We substitute (A.10) in (A.9) and use definition (A.11) in the first term in the right hand side of (A.9) to obtain,

$$
\begin{aligned}
\left\|k_{*}-k^{n-1}\right\|_{\mathcal{K}}^{2}-\left\|k_{*}-k^{n}\right\|_{\mathcal{K}}^{2} & >-2\left[d^{\eta}-\mathcal{L}\left(s_{F}^{n}\right)\right]^{T} C^{-1 / 2} A C^{-1 / 2}\left[d^{\eta}-\mathcal{L}\left(s_{r}^{n}\right)\right] \\
& +2\left[d^{\eta}-\mathcal{L}\left(s_{F}^{n}\right)\right]^{T} C^{-1 / 2} A^{2} C^{-1 / 2}\left[d^{\eta}-\mathcal{L}\left(s_{F}^{n}\right)\right] .
\end{aligned}
$$

Moreover, we note that

$$
2\left[d^{\eta}-\mathcal{L}\left(s_{F}^{n}\right)\right]^{T} C^{-1 / 2} A C^{-1 / 2}\left[d^{\eta}-\mathcal{L}\left(s_{r}^{n}\right)\right] \leq 2\left\|A C^{-1 / 2}\left[d^{\eta}-\mathcal{L}\left(s_{F}^{n}\right)\right]\right\|\left\|d^{\eta}-\mathcal{L}\left(s_{r}^{n}\right)\right\|_{C^{-1}} .
$$

This inequality, applied to (A.12), yields

$$
\begin{array}{r}
\left\|k_{*}-k^{n-1}\right\|_{\mathcal{K}}^{2}-\left\|k_{*}-k^{n}\right\|_{\mathcal{K}}^{2} \\
>2\left\|A C^{-1 / 2}\left[d^{\eta}-\mathcal{L}\left(s_{F}^{n}\right)\right]\right\|\left[\left\|A C^{-1 / 2}\left[d^{\eta}-\mathcal{L}\left(s_{F}^{n}\right)\right]\right\|-\left\|d^{\eta}-\mathcal{L}\left(s_{r}^{n}\right)\right\|_{C^{-1}}\right] .
\end{array}
$$

On the other hand, is easy to see that

$$
\left\|A C^{-1 / 2}\left[d^{\eta}-\mathcal{L}\left(s_{F}^{n}\right)\right]\right\| \geq \frac{1}{\left\|A^{-1}\right\|}\left\|C^{-1 / 2}\left[d^{\eta}-\mathcal{L}\left(s_{F}^{n}\right)\right]\right\|=\frac{1}{\left\|A^{-1}\right\|}\left\|d^{\eta}-\mathcal{L}\left(s_{F}^{n}\right)\right\|_{C^{-1}} .
$$

Furthermore, from (A.11) and (3.17) it follows

$$
\begin{aligned}
\left\|A^{-1}\right\| \leq\left\|C^{-1 / 2}\left[R^{n}+C\right] C^{-1 / 2}\right\|= & \left\|C^{-1 / 2} R^{n} C^{-1 / 2}+I\right\| \\
& \leq\|\mathcal{L}\|^{2} C_{1}^{2}\left\|C^{-1}\right\|+1 .
\end{aligned}
$$

Combining (A.15), (A.16) and (3.32), we find

$$
\left\|A C^{-1 / 2}\left[d^{\eta}-\mathcal{L}\left(s_{F}^{n}\right)\right]\right\|>\frac{1}{\|\mathcal{L}\|^{2} C_{1}^{2}\left\|C^{-1}\right\|+1}\left\|d^{\eta}-\mathcal{L}\left(s_{F}^{n}\right)\right\|_{C^{-1}}=\rho\left\|d^{\eta}-\mathcal{L}\left(s_{F}^{n}\right)\right\|_{C^{-1}}(\mathrm{~A}
$$


Then, (A.14) becomes

$$
\begin{array}{r}
\left\|k_{*}-k^{n-1}\right\|_{\mathcal{K}}^{2}-\left\|k_{*}-k^{n}\right\|_{\mathcal{K}}^{2} \\
>2\left\|A C^{-1 / 2}\left[d^{\eta}-\mathcal{L}\left(s_{F}^{n}\right)\right]\right\|\left[\rho\left\|d^{\eta}-\mathcal{L}\left(s_{F}^{n}\right)\right\| C_{C^{-1}}-\left\|d^{\eta}-\mathcal{L}\left(s_{r}^{n}\right)\right\|_{C^{-1}}\right],
\end{array}
$$

and by using hypothesis (3.33)

$$
\begin{array}{r}
\left\|k_{*}-k^{n-1}\right\|_{\mathcal{K}}^{2}-\left\|k_{*}-k^{n}\right\|_{\mathcal{K}}^{2} \\
>2 \rho\left[\frac{a-1}{a}\right]\left\|A C^{-1 / 2}\left[d^{\eta}-\mathcal{L}\left(s_{F}^{n}\right)\right]\right\|\left\|d^{\eta}-\mathcal{L}\left(s_{F}^{n}\right)\right\|_{C^{-1}}
\end{array}
$$

An additional application of (A.17) yields (3.35).

\section{A.2 Proof of Theorem 2}

First we show that the sequence is monotone decreasing. Since $k_{*}$ is a solution to the NFPIP, let $s_{*}$ be such that $G\left(k_{*}, s_{*}\right)=0$ and $\mathcal{L}\left(s_{*}\right)=d$. On the other hand, note from $(3.36)$ that $\bar{k} \in B\left(\delta, k_{*}\right.$ ), then from (A2) (for $k=\hat{k}=k_{*}, \tilde{k}=\bar{k}$ ) it follows that

$$
\left\|\mathcal{L}\left(s_{r}\right)\right\| \leq C_{2}\left\|k_{*}-\bar{k}\right\|_{\mathcal{K}}\left\|\mathcal{L}\left(s_{*}\right)-\mathcal{L}\left(s_{F}^{1}\right)\right\|,
$$

where $s_{r}$ satisfies $(3.31)$ :

$$
D_{s} G\left(\bar{k}, s_{F}^{1}\right) s_{r}=-D_{k} G\left(\bar{k}, s_{F}^{1}\right)\left[k_{*}-\bar{k}\right]-D_{s} G\left(\bar{k}, s_{F}^{1}\right)\left[s_{*}-s_{F}^{1}\right]
$$

Then, it is not difficult to see that

$$
\begin{aligned}
\left\|\mathcal{L}\left(s_{r}\right)\right\|_{C^{-1}} \leq\left\|C^{-1}||^{1 / 2}|| \mathcal{L}\left(s_{r}\right)\right\| & \leq\left\|C^{-1}||^{1 / 2} C_{2}\right\| k_{*}-\bar{k}\left\|_{\mathcal{K}}\right\| \mathcal{L}\left(s_{*}\right)-\mathcal{L}\left(s_{F}^{1}\right) \| \\
& \leq \alpha^{1 / 2} C_{2}\left\|k_{*}-\bar{k}\right\|_{\mathcal{K}}\left\|\mathcal{L}\left(s_{*}\right)-\mathcal{L}\left(s_{F}^{1}\right)\right\|_{C^{-1}}
\end{aligned}
$$

If we perform the change of variables $s_{t}=s_{r}+s_{*}$, it follows that (A.21) becomes

$$
0=D_{k} G\left(\bar{k}, s_{F}^{1}\right)\left[k_{*}-\bar{k}\right]+D_{s} G\left(\bar{k}, s_{F}^{1}\right)\left[s_{t}-s_{F}^{1}\right]
$$

which must be solved for $s_{t}$. The previous change of variables is substituted in (A.22) to obtain

$$
\left\|d-\mathcal{L}\left(s_{t}\right)\right\|_{C^{-1}} \leq a_{2} \alpha^{1 / 2} C_{2}\left\|d-\mathcal{L}\left(s_{F}^{1}\right)\right\|_{C^{-1}} \leq \frac{\rho}{2}\left\|d-\mathcal{L}\left(s_{F}^{1}\right)\right\|_{C^{-1}},
$$


where we have used (3.36) and the fact that $\mathcal{L}\left(s_{*}\right)=d$. From (A.23) and (A.24) we realize that the assumptions of the previous lemma are satisfied $(a=2)$. Then $k^{1}$, given by (3.21), for $d^{\eta}=d$ (see Remark 1) satisfies

$$
\left\|k_{*}-k^{0}\right\|_{\mathcal{K}}^{2}-\left\|k_{*}-k^{1}\right\|_{\mathcal{K}}^{2}>\rho^{2}\left\|d-\mathcal{L}\left(s_{F}^{0}\right)\right\|_{C^{-1}}^{2}
$$

Therefore, $\delta>\left\|k_{*}-k^{0}\right\|_{\mathcal{K}}^{2}>\left\|k_{*}-k^{1}\right\|_{\mathcal{K}}^{2}$ and we can apply the same argument inductively to show that

$$
\left\|k_{*}-k^{n-1}\right\|_{\mathcal{K}}^{2}>\left\|k_{*}-k^{n}\right\|_{\mathcal{K}}^{2}
$$

for all $n$. For this reason we may define

$$
\alpha=\lim _{n \rightarrow \infty}\left\|k_{*}-k^{n}\right\|_{\mathcal{K}}
$$

We now prove that $k^{n}$ is Cauchy. Let $m>q$ and consider $l \in[q, m]$ such that

$$
\left\|d-\mathcal{L}\left(s_{F}^{l}\right)\right\|_{C^{-1}} \leq\left\|d-\mathcal{L}\left(s_{F}^{n}\right)\right\|_{C^{-1}}
$$

for all $n \in[q, m]$. Note from (3.21) that

$$
\begin{array}{r}
\left\langle k_{*}-k^{l-1}, k^{n}-k^{n-1}\right\rangle_{\mathcal{K}}=\left\langle k_{*}-k^{l-1}, \gamma^{n}[R+C]^{-1}\left[d-\mathcal{L}\left(s_{F}^{n}\right)\right]\right\rangle_{\mathcal{K}} \\
=\sum_{j m}^{M}[R+C]_{j m}^{-1}\left[d-\mathcal{L}\left(s_{F}^{n}\right)\right]_{j}\left\langle k_{*}-k^{l-1}, \gamma_{m}^{n}\right\rangle_{\mathcal{K}} .
\end{array}
$$

By the definition of $\gamma_{m}^{n}((3.14),(3.19))$ we know that

$$
\left\langle k_{*}-k^{l-1}, \gamma_{m}^{n}\right\rangle_{\mathcal{K}}=\mathcal{L}_{m}(\Delta s),
$$

where $\Delta s$ satisfies

$$
0=D_{k} G\left(k^{n-1}, s_{F}^{n}\right)\left[k_{*}-k^{l-1}\right]+D_{s} G\left(k^{n-1}, s_{F}^{n}\right) \Delta s .
$$

On the other hand, let $s_{r}^{n}$ be the solution to

$$
0=D_{k} G\left(k^{n-1}, s_{F}^{n}\right)\left[k_{*}-k^{n-1}\right]+D_{s} G\left(k^{n-1}, s_{F}^{n}\right)\left[s_{r}^{n}-s_{F}^{n}\right]
$$


We subtract the previous equation from (A.31) to show that $\Delta s$ satisfies

$$
0=D_{k} G\left(k^{n-1}, s_{F}^{n}\right)\left[k^{l-1}-k^{n-1}\right]+D_{s} G\left(k^{n-1}, s_{F}^{n}\right)\left[s_{r}^{n}-\Delta s-s_{F}^{n}\right] .
$$

Observe that $k^{l-1}, k^{n-1} \in B\left(\delta / 2, k_{*}\right)$ by (A.26). Then, $\left\|k^{l-1}-k^{n-1}\right\|_{\mathcal{K}}<\delta$, and we may apply assumption (A2) for $k=k^{l-1}, \tilde{k}=k^{n-1}, s=s_{F}^{l}$ and $\tilde{s}=s_{F}^{n}$. Then,

$$
\begin{array}{r}
\left\|\mathcal{L}\left(s_{F}^{l}\right)-\mathcal{L}\left(\left[s_{r}^{n}-\Delta s\right]\right)\right\|_{C^{-1}} \leq \alpha^{1 / 2} C_{2}\left\|k^{l-1}-k^{n-1}\right\| \mathcal{K}\left\|\mathcal{L}\left(s_{F}^{l}\right)-\mathcal{L}\left(s_{F}^{n}\right)\right\|_{C^{-1}} \\
\leq C_{2} \alpha^{1 / 2} \delta\left\|\mathcal{L}\left(s_{F}^{l}\right)-\mathcal{L}\left(s_{F}^{n}\right)\right\|_{C^{-1}}
\end{array}
$$

where we applied the same argument that we used to obtain (A.22). We now substitute (A.30) in (A.29), use (A.34) and the triangle inequality to obtain

$$
\begin{array}{r}
\left|\left\langle k_{*}-k^{l-1}, k^{n}-k^{n-1}\right\rangle_{\mathcal{K}}\right| \leq\left\|\left[d-\mathcal{L}\left(s_{F}^{n}\right)\right][R+C]^{-1} C^{1 / 2}\right\|\|\mathcal{L}(\Delta s)\|_{C^{-1}} \\
\leq C_{2} \alpha^{1 / 2} \delta\left\|\left[d-\mathcal{L}\left(s_{F}^{n}\right)\right][R+C]^{-1} C^{1 / 2}\right\|\left\|\mathcal{L}\left(s_{F}^{l}\right)-\mathcal{L}\left(s_{F}^{n}\right)\right\|_{C^{-1}} \\
+\left\|\left[d-\mathcal{L}\left(s_{F}^{n}\right)\right][R+C]^{-1} C^{1 / 2}\right\|\left\|\mathcal{L}\left(s_{r}^{n}-s_{F}^{l}\right)\right\|_{C^{-1}}
\end{array}
$$

Applying the triangle inequality again we find

$$
\begin{array}{r}
\left|\left\langle k_{*}-k^{l-1}, k^{n}-k^{n-1}\right\rangle_{\mathcal{K}}\right| \leq\left\|\left[d-\mathcal{L}\left(s_{F}^{n}\right)\right][R+C]^{-1} C^{1 / 2}\right\|\left[C_{2} \delta \alpha^{1 / 2}\left\|d-\mathcal{L}\left(s_{F}^{n}\right)\right\|_{C^{-1}}\right. \\
\left.+C_{2} \delta \alpha^{1 / 2}\left\|\mathcal{L}\left(s_{F}^{l}\right)-d\right\|_{C^{-1}}+\left\|\mathcal{L}\left(s_{r}^{n}\right)-d\right\|\left\|_{C^{-1}}+\right\| d-\mathcal{L}\left(s_{F}^{l}\right) \|_{C^{-1}}\right] .
\end{array}
$$

In addition, by definition of $s_{r}^{n}$ (A.32) and since $\left\|k_{*}-k^{n-1}\right\|_{\mathcal{K}}<\delta$, we apply (A2) one more time to derive

$$
\left\|d-\mathcal{L}\left(s_{r}^{n}\right)\right\|_{C^{-1}} \leq\left. C_{2} \alpha^{1 / 2}\left\|k_{*}-k^{n-1}\right\|\right|_{\mathcal{K}}\left\|d-\mathcal{L}\left(s_{F}^{n}\right)\right\|_{C^{-1}} \leq C_{2} \alpha^{1 / 2} \delta\left\|d-\mathcal{L}\left(s_{F}^{n}\right)\right\|_{C^{-1}}(
$$

where we have used the fact that $\mathcal{L}\left(s_{*}\right)=d\left(k_{*}\right.$ is a solution to the NF-PIP). Expression (A.37) is substituted in (A.36). Then, (A.17) and (A.28) yields

$$
\left|\left\langle k_{*}-k^{l-1}, k^{n}-k^{n-1}\right\rangle_{\mathcal{K}}\right| \leq\left\|A C^{-1 / 2}\left[d-\mathcal{L}\left(s_{F}^{n}\right)\right]||\left(3 C_{2} \delta \alpha^{1 / 2}+1\right)\right\| d-\mathcal{L}\left(s_{F}^{n}\right) \|_{C^{-1}}
$$

On the other hand, as we indicated earlier, the previous lemma applies for $a=2$, so we can use expression (A.19) to find

$$
\frac{1}{\rho}\left[\left\|k_{*}-k^{n-1}\right\|_{\mathcal{K}}^{2}-\left\|k_{*}-k^{n}\right\|_{\mathcal{K}}^{2}\right]>\left\|A C^{-1 / 2}\left[d-\mathcal{L}\left(s_{F}^{n}\right)\right]\right\|\left\|d-\mathcal{L}\left(s_{F}^{n}\right)\right\|_{C^{-1}}
$$


Then we combine (A.38) and (A.39) to obtain

$$
\left|\left\langle k_{*}-k^{l-1}, k^{n}-k^{n-1}\right\rangle_{\mathcal{K}}\right| \leq \frac{\left(3 C_{2} \alpha^{1 / 2} \delta+1\right)}{\rho}\left[\left\|k_{*}-k^{n-1}\right\|_{\mathcal{K}}^{2}-\left\|k_{*}-k^{n}\right\|_{\mathcal{K}}^{2}\right]
$$

Furthermore, we define $e_{i}=k_{*}-k^{i}$. So, for $l, q, m$ as before, it is not difficult to see that

$$
\left\langle e_{l-1}-e_{q-1}, e_{l-1}\right\rangle_{\mathcal{K}}=\sum_{n=q}^{l-1}\left\langle k^{n}-k^{n-1}, k^{l-1}-k_{*}\right\rangle_{\mathcal{K}}
$$

Then, from (A.40)

$$
\begin{aligned}
\left|\left\langle e_{l-1}-e_{q-1}, e_{l-1}\right\rangle_{\mathcal{K}}\right| \leq & \frac{\left(3 C_{2} \delta \alpha^{1 / 2}+1\right)}{\rho} \sum_{n=q}^{l-1}\left(\left\|k_{*}-k^{n-1}\right\|_{\mathcal{K}}^{2}-\left\|k_{*}-k^{n}\right\|_{\mathcal{K}}^{2}\right) \\
& =\frac{\left(3 C_{2} \delta \alpha^{1 / 2}+1\right)}{2 \rho}\left(\left\|k_{*}-k^{q-1}\right\|_{\mathcal{K}}^{2}-\left\|k_{*}-k^{l-1}\right\|_{\mathcal{K}}^{2}\right),
\end{aligned}
$$

so, from (A.27) $\left|\left\langle e_{l-1}-e_{q-1}, e_{l-1}\right\rangle_{\mathcal{K}}\right| \rightarrow \alpha-\alpha=0$ as $q, l \rightarrow \infty$. With the same argument it follows that $\left\|e_{l}-e_{q}\right\|_{\mathcal{K}}^{2} \rightarrow 0$ and $\left\|e_{l}-e_{m}\right\|_{\mathcal{K}}^{2} \rightarrow 0$ as $m, q, l \rightarrow \infty$. Finally, since $\left\|k^{m}-k^{q}\right\|_{\mathcal{K}}^{2}=$ $\left\|e_{m}-e_{q}\right\|_{\mathcal{K}}^{2} \leq 2\left\|e_{m}-e_{l}\right\|_{\mathcal{K}}^{2}+2\left\|e_{l}-e_{q}\right\|_{\mathcal{K}}^{2}$, we have that $k^{n}$ is a Cauchy sequence.

Then, by completeness of $\mathcal{K}, k^{n}$ has a convergent subsequence also denoted by $k^{n}$. Let us define $\hat{k}=\lim _{n \rightarrow \infty} k^{n}$. Let, $\hat{s}$ be the solution to $G(\hat{k}, \hat{s})=0$. Since $G$ satisfies (F1)-(F3), by the Implicit Function Theorem (IFT), there exists a neighborhood $U$ of $\hat{k}$ such that $G(k, s(k))=0$ for all $k \in U$, where $s: \mathcal{U} \rightarrow \mathcal{S}$ is a continuous function. Since $\left\|k^{n}-\hat{k}\right\|_{\mathcal{K}} \rightarrow 0$ as $n \rightarrow \infty$, we may take $n$ sufficiently large such that $k^{n} \in U$. Therefore, as $n \rightarrow \infty$, by continuity of $s$ (with respect $k$ ), $s_{F}^{n+1}=s\left(k^{n}\right) \rightarrow s(\hat{k})=\hat{s}$. By linearity, we also have that $\mathcal{L}\left(s_{F}^{n+1}\right) \rightarrow \mathcal{L}(\hat{s})$. However, from (A.25), it follows

$$
\begin{array}{r}
\sum_{n=1}^{l}\left\|d-\mathcal{L}\left(s_{F}^{n}\right)\right\|^{2}<\frac{1}{\rho^{2}} \sum_{n=1}^{l}\left(\left\|k_{*}-k^{n-1}\right\|_{\mathcal{K}}^{2}-\left\|k_{*}-k^{n}\right\|_{\mathcal{K}}^{2}\right) \\
=\frac{1}{\rho^{2}}\left(\left\|k_{*}-k^{0}\right\|_{\mathcal{K}}^{2}-\left\|k_{*}-k^{l}\right\|_{\mathcal{K}}^{2}\right) .
\end{array}
$$

Then, when $l \rightarrow \infty$ it follows

$$
\sum_{n=1}^{\infty}\left\|d-\mathcal{L}\left(s_{F}^{n}\right)\right\|^{2}<\frac{1}{\rho^{2}}\left(\left\|k_{*}-k^{0}\right\|_{\mathcal{K}}^{2}-\alpha\right)<\infty
$$

and so $\left\|d-\mathcal{L}\left(s_{F}^{n}\right)\right\|^{2} \rightarrow 0$. Therefore, as $n \rightarrow \infty, \mathcal{L}\left(s_{F}^{n}\right) \rightarrow d$ and since we already proved 
that $\mathcal{L}\left(s_{F}^{n}\right) \rightarrow \mathcal{L}(\hat{s})$, it now follows $d=\mathcal{L}(\hat{s})$. We conclude that $\hat{k}$ is a solution to the NF-PIP.

In contrast to the proof of Theorem 2.2 in [32], in the previous theorem an acceptable distance between the initial guess and a solution to the NF-PIP depends not only on the nonlinearity condition (Assumption 2), but also on the norm of the measurement functional $\mathcal{L}$, and the prior error covariance matrix $C$.

\section{A.3 Proof of Theorem 3}

Since $k_{*}$ is a solution to the NF-PIP, let $s_{*} \in \mathcal{S}$ be such that $G\left(k_{*}, s_{*}\right)=0$ and $\mathcal{L}\left(s_{*}\right)=d$. Assume $\eta>0$ is fixed. Let $s_{r}^{1}$ be the solution to

$$
D_{s} G\left(\bar{k}, s_{F}^{1}\right)\left[s_{r}^{1}-s_{*}\right]=G_{r}\left(s_{*}-s_{F}^{1}, k_{*}-\bar{k}\right) .
$$

Since $\left\|k_{*}-\bar{k}\right\|_{\mathcal{K}} \leq \delta / 2<\delta$, we apply Assumption (A2) (for $\hat{k}=k=k_{*}$ and $\tilde{k}=\bar{k}$ ) to obtain

$$
\left\|\mathcal{L}\left(s_{*}\right)-\mathcal{L}\left(s_{r}^{1}\right)\right\|_{C^{-1}} \leq C_{2} \alpha^{1 / 2}\left\|k_{*}-\bar{k}\right\|_{\mathcal{K}}\left\|\mathcal{L}\left(s_{*}\right)-\mathcal{L}\left(s_{F}^{1}\right)\right\|_{C^{-1}}
$$

Note now that

$$
\begin{array}{r}
\left\|d^{\eta}-\mathcal{L}\left(s_{r}^{1}\right)\right\|_{C^{-1}} \leq\left\|d^{\eta}-\mathcal{L}\left(s_{*}\right)\right\|_{C^{-1}}+\left\|\mathcal{L}\left(s_{*}\right)-\mathcal{L}\left(s_{r}^{1}\right)\right\|_{C^{-1}} \\
\leq \eta+C_{2} \alpha^{1 / 2}\left\|k_{*}-\bar{k}\right\|_{\mathcal{K}}\left\|\mathcal{L}\left(s_{*}\right)-\mathcal{L}\left(s_{F}^{1}\right)\right\|_{C^{-1}} \\
\leq \eta+C_{2} \alpha^{1 / 2}\left\|k_{*}-\bar{k}\right\|_{\mathcal{K}}\left[\left\|d^{\eta}-\mathcal{L}\left(s_{*}\right)\right\|_{C^{-1}}+\left\|d^{\eta}-\mathcal{L}\left(s_{F}^{1}\right)\right\|_{C^{-1}}\right] \\
\leq\left[1+C_{2} \alpha^{1 / 2}\left\|k_{*}-\bar{k}\right\|_{\mathcal{K}}\right] \eta+C_{2} \alpha^{1 / 2}\left\|k_{*}-\bar{k}\right\|_{\mathcal{K}}\left\|d^{\eta}-\mathcal{L}\left(s_{F}^{1}\right)\right\|_{C^{-1}}
\end{array}
$$

where we used (A.46) and the hypothesis on the noise level $\left(\left\|d^{\eta}-\mathcal{L}\left(s_{*}\right)\right\|_{C^{-1}} \leq \eta\right)$. On the other hand, by the discrepancy principle, before we terminate the IRBS $\tau \eta<\| d^{\eta}-$ $\mathcal{L}\left(s_{F}^{1}\right) \|_{C^{-1}}$ and so

$$
\begin{aligned}
& \left\|d^{\eta}-\mathcal{L}\left(s_{r}^{1}\right)\right\|_{C^{-1}} \leq \frac{1}{\tau}\left[1+C_{2} \alpha^{1 / 2}\left\|k_{*}-\bar{k}\right\| \|_{\mathcal{K}}\right]\left\|d^{\eta}-\mathcal{L}\left(s_{F}^{1}\right)\right\|_{C^{-1}} \\
& \quad+C_{2} \alpha^{1 / 2}\left\|k_{*}-\bar{k}\right\|_{\mathcal{K}}\left\|d^{\eta}-\mathcal{L}\left(s_{F}^{1}\right)\right\|_{C^{-1}}=\frac{\rho}{a}\left\|d^{\eta}-\mathcal{L}\left(s_{F}^{1}\right)\right\|_{C^{-1}}
\end{aligned}
$$

for

$$
a=\frac{\rho \tau}{1+(1+\tau) C_{2} \alpha^{1 / 2}\left\|k_{*}-k^{0}\right\|_{\mathcal{K}}} .
$$


Then, since $\bar{k} \in B\left(\delta / 2, k_{*}\right)$ we may apply Lemma 2 to obtain that $k_{\eta}^{1}$, defined by $(3.21)$, satisfies

$$
\left\|k_{*}-\bar{k}\right\|_{\mathcal{K}}^{2}-\left\|k_{*}-k_{\eta}^{1}\right\|_{\mathcal{K}}^{2}>2 \rho^{2}\left[\frac{a-1}{a}\right]\left\|d^{\eta}-\mathcal{L}\left(s_{F}^{1}\right)\right\|_{C^{-1}}^{2}
$$

(we have added the subscript $\eta$ to emphasize that $k_{\eta}^{1}$ depends in this case of $d^{\eta}$ ). From (3.37) note that

$$
\left\|k_{*}-k^{0}\right\|_{\mathcal{K}}<\frac{\rho \tau-1}{4 \alpha^{1 / 2} C_{2}(1+\tau)}<\frac{\rho \tau-1}{\alpha^{1 / 2} C_{2}(1+\tau)}
$$

and so

$$
1+\alpha^{1 / 2} C_{2}(1+\tau)\left\|k_{*}-k^{0}\right\|_{\mathcal{K}}<\rho \tau
$$

Then, (A.49) implies $a>1$. Therefore, $\delta / 2>\left\|k_{*}-\bar{k}\right\|_{\mathcal{K}}^{2}>\left\|k_{*}-k_{\eta}^{1}\right\|_{\mathcal{K}}^{2}$. By applying this methodology inductively, we show that $k_{\eta}^{n}$ given by (3.21) satisfies

$$
\left\|k_{*}-k_{\eta}^{n-1}\right\|_{\mathcal{K}}^{2}>\left\|k_{*}-k_{\eta}^{n}\right\|_{\mathcal{K}}^{2}
$$

as long as $\tau \eta<\left\|d^{\eta}-\mathcal{L}\left(s_{F}^{n}\right)\right\|_{C^{-1}}$ and so the sequence $k_{\eta}^{n}$ is monotone decreasing. Let us assume that for $n=n(\eta)$,

$$
\left\|d^{\eta}-\mathcal{L}\left(s_{F}^{n}\right)\right\|_{C^{-1}} \leq \tau \eta
$$

From (A.50) it follows that

$$
\sum_{n=1}^{n(\eta)-1}\left(\left\|k_{*}-k_{\eta}^{n-1}\right\|_{\mathcal{K}}^{2}-\left\|k_{*}-k_{\eta}^{n}\right\|_{\mathcal{K}}^{2}\right)>2 \rho^{2}\left[\frac{a-1}{a}\right] \sum_{n=1}^{n(\eta)-1}\left\|d^{\eta}-\mathcal{L}\left(s_{F}^{n}\right)\right\|_{C^{-1}}^{2}
$$

Reducing some terms in the left hand side and using the fact that $\tau \eta<\left\|d^{\eta}-\mathcal{L}\left(s_{F}^{n}\right)\right\|_{C^{-1}}$, we find

$$
\infty>\left\|k_{*}-\bar{k}\right\|_{\mathcal{K}}^{2} \geq\left\|k_{*}-\left.\bar{k}\right|_{\mathcal{K}} ^{2}-\right\| k_{*}-k_{\eta}^{n(\eta)-1} \|_{\mathcal{K}}^{2}>2 \rho^{2}\left[\frac{a-1}{a}\right] \tau^{2} \eta^{2}(n(\eta)-1) .
$$

This inequality shows that, given $\eta>0$ fixed, the discrepancy principle terminates the IRBS after a finite number $n(\eta)$ of iterations. We now observe from (3.37) that $\left\|k_{*}-k^{0}\right\|_{\mathcal{K}}<$ $\rho /\left(2 \alpha^{1 / 2} C_{2}\right)$. Therefore, Theorem 2 can be applied where now $k^{n}$ is the sequence given by (3.21) with $d^{\eta}$ replaced by $d$. 
We now prove by induction that for all $m$ fixed, $k_{\eta}^{m} \rightarrow k^{m}$ as $\eta \rightarrow 0$. For $m=0$ the result follows trivially. Let us assume that $\left\|k_{\eta}^{m-1}-k^{m-1}\right\|_{\mathcal{K}} \rightarrow 0$ as $\eta \rightarrow 0$. Let $s_{F}^{m}$ and $s_{F, \eta}^{m}$ be such that $G\left(k^{m-1}, s_{F}^{m}\right)=0=G\left(k_{\eta}^{m-1}, s_{F, \eta}^{m}\right)$. By definition,

$$
k_{\eta}^{m}-k^{m}=k_{\eta}^{m-1}-k^{m-1}+\left[\gamma^{m}\right]^{T}\left[R^{m}+C\right]^{-1}\left[d^{\eta}-\mathcal{L}\left(s_{F, \eta}^{m}\right)-d+\mathcal{L}\left(s_{F}^{m}\right)\right] .
$$

Therefore,

$$
\begin{array}{r}
\left\|k_{\eta}^{m}-k^{m}\right\|_{\mathcal{K}} \leq\left\|k_{\eta}^{m-1}-k^{m-1}\right\|_{\mathcal{K}} \\
+\left\|\gamma^{m}\right\|_{\mathcal{K}}\left\|\left[R^{m}+C\right]^{-1} C^{1 / 2}\right\|\left[\eta+\left\|\mathcal{L}\left(s_{F, \eta}^{m}\right)-\mathcal{L}\left(s_{F}^{m}\right)\right\|_{C^{-1}}\right] .
\end{array}
$$

We now use the inductive hypothesis, the IFT and the linearity of $\mathcal{L}$ to conclude that $k_{\eta}^{m} \rightarrow k^{m}$ as $\eta \rightarrow 0$

Now we show that if $n(\eta)$ remains bounded for all $\eta>0, k_{\eta}^{n}$ converges to $k^{n}$ as $\eta \rightarrow 0$ and that $k^{n}$ is a solution to the NF-PIP.

$$
\left\|d-\mathcal{L}\left(s_{F}^{m}\right)\right\|_{C^{-1}} \leq \eta+\left\|d^{\eta}-\mathcal{L}\left(s_{F, \eta}^{m}\right)\right\|_{C^{-1}}+\left\|\mathcal{L}\left(s_{F, \eta}^{m}\right)-\mathcal{L}\left(s_{F}^{m}\right)\right\|_{C^{-1}}
$$

for all $m$. In particular, for the stopping index $n=n(\eta)($ A.54), it follows that

$$
\left\|d-\mathcal{L}\left(s_{F}^{n}\right)\right\|_{C^{-1}} \leq \eta+\tau \eta+\left\|\mathcal{L}\left(s_{F, \eta}^{n}\right)-\mathcal{L}\left(s_{F}^{n}\right)\right\|_{C^{-1}}
$$

Therefore, since we already showed that $k_{\eta}^{n} \rightarrow k^{n}$ as $\eta \rightarrow 0$, we use the same argument as before to obtain that $\left\|d-\mathcal{L}\left(s_{F}^{n}\right)\right\|=0$. Then $k_{\eta}^{n}$ converges to a solution to the NF-PIP as $\eta \rightarrow 0$.

Now we consider the case where $n(\eta) \rightarrow \infty$ as $\eta \rightarrow 0$. Let $k$ be the limit of $k^{n}$, the sequence with exact data which exists by Theorem 2 and is a solution to the NF-PIP. Then we may take $n$ large enough such that

$$
\left\|k-k^{n}\right\|_{\mathcal{K}}<\frac{\rho \tau-1}{4 \alpha^{1 / 2} C_{2}(1+\tau)}
$$

Thus,

$$
\left\|k-k_{*}\right\|_{\mathcal{K}} \leq\left\|k-k^{n}\right\|_{\mathcal{K}}+\left\|k^{n}-k_{*}\right\|_{\mathcal{K}} \leq \frac{\rho \tau-1}{4 \alpha^{1 / 2} C_{2}(1+\tau)}+\left\|\bar{k}-k_{*}\right\|_{\mathcal{K}}
$$


where we have used the fact that $k^{n}$ is monotone decreasing. Then, from (3.37)

$$
\begin{array}{r}
\|k-\bar{k}\|_{\mathcal{K}} \leq\left\|k-k_{*}\right\|_{\mathcal{K}}+\left\|k_{*}-\bar{k}\right\|_{\mathcal{K}} \leq \frac{\rho \tau-1}{4 \alpha^{1 / 2} C_{2}(1+\tau)}+2\left\|\bar{k}-k_{*}\right\|_{\mathcal{K}} \\
<\frac{3}{4} \frac{\rho \tau-1}{\alpha^{1 / 2} C_{2}(1+\tau)}<\frac{\rho \tau-1}{C_{2} \alpha^{1 / 2}(1+\tau)}
\end{array}
$$

Then, by the argument used above, expression (A.53) follows with $k_{*}$ replaced by $k$. On the other hand, given $\epsilon>0$, since $k^{n} \rightarrow k$, we may take $m(\epsilon)$ such that if $m \geq m(\epsilon)$, then $\left\|k-k^{m}\right\|_{\mathcal{K}} \leq \frac{\epsilon}{2}$. In addition, since $n(\eta) \rightarrow \infty$ as $\eta \rightarrow 0$, there exists $\delta>0$, such that $n(\eta)>m(\epsilon)$ whenever $\eta<\delta$. Furthermore, we note from A.57 (with $n$ replaced by $m(\epsilon)$ ), that there exists $\delta_{2}$ such that, if $\eta<\delta_{2},\left\|k_{\eta}^{m(\epsilon)}-k^{m(\epsilon)}\right\|_{\mathcal{K}} \leq \frac{\epsilon}{2}$. Then if $\eta<\min \left(\delta, \delta_{2}\right)$,

$$
\left\|k_{\eta}^{n(\eta)}-k\right\|_{\mathcal{K}} \leq\left\|k_{\eta}^{m(\epsilon)}-k\right\|_{\mathcal{K}} \leq\left\|k_{\eta}^{m(\epsilon)}-k^{m(\epsilon)}\right\|_{\mathcal{K}}+\left\|k-k^{m(\epsilon)}\right\|_{\mathcal{K}} \leq \epsilon
$$

Thus, as $n(\eta) \rightarrow \infty$ for $\eta \rightarrow 0, k_{\eta}^{n} \rightarrow k$ where $k$ is a solution to the NF-PIP. 


\section{Appendix B}

\section{Derivation of the IRBS for Two-Phase Flow}

\section{B.1 Reduction of the Linearized Case}

From definition (6.48) and equations (6.26)-(6.31) we know that

$$
\begin{aligned}
& q_{F}^{l, n}=\omega_{P}^{l} \lambda_{F}^{n} e^{Y^{n-1}}\left[P_{b h}^{l}-p_{F}^{n}\left(x_{P}^{l}\right)\right], \\
& \mathbf{u}_{F}^{n}=\quad-\lambda_{F}^{n} e^{Y^{n-1}} \nabla p_{F}^{n}, \\
& Q^{l}=\omega_{I}^{l} e^{Y^{n-1}} \lambda_{F}^{n}\left[p_{b h, F}^{l, n}-p_{F}^{n}\left(x_{I}^{l}\right)\right] .
\end{aligned}
$$

Using again definition (6.48) it is straightforward tho observe that

$$
\begin{array}{r}
D_{\mathcal{P}} G\left(\mathcal{P}^{n-1}, \mathcal{S}_{F}^{n}\right)\left[\mathcal{P}^{n}-\mathcal{P}^{n-1}\right]+D_{s} G\left(\mathcal{P}^{n-1}, \mathcal{S}_{F}^{n}\right)\left[\mathcal{S}-\mathcal{S}_{F}^{n}\right] \\
=\phi_{F}^{n} c\left(s_{F}^{n}\right) \frac{\partial\left[p-p_{F}^{n}\right]}{\partial t}+\phi_{F}^{n}\left[c_{w}-c_{o}\right] \frac{\partial p_{F}^{n}}{\partial t}\left[s-s_{F}^{n}\right] \\
+\left[\phi_{r}-\phi_{r}^{n-1}\right] c\left(s_{F}^{n}\right) \frac{\partial p_{F}^{n}}{\partial t}+\nabla \cdot\left[\mathbf{u}-\mathbf{u}_{F}^{n}\right]-\sum_{l=1}^{N_{p}}\left[q-q_{F}^{n}\right] \delta\left(x-x_{p}^{l}\right),
\end{array}
$$




$$
\begin{aligned}
& D_{\mathcal{P}} G_{w}\left(\mathcal{P}^{n-1}, \mathcal{S}_{F}^{n}\right)\left[\mathcal{P}^{n}-\mathcal{P}^{n-1}\right]+D_{s} G_{w}\left(\mathcal{P}^{n-1}, \mathcal{S}_{F}^{n}\right)\left[\mathcal{S}-\mathcal{S}_{F}^{n}\right] \\
& =\phi_{F}^{n}\left(c_{r}+c_{w}\right) s_{F}^{n} \frac{\partial\left[p-p_{F}^{n}\right]}{\partial t}+c_{r} \phi_{r}^{n-1}\left[p-p_{F}^{n}\right] \frac{\partial s_{F}^{n}}{\partial t}+\phi_{F}^{n}\left(c_{r}+c_{w}\right)\left[s-s_{F}^{n}\right] \frac{\partial p_{F}^{n}}{\partial t} \\
& +\phi_{F}^{n} \frac{\partial\left[s-s_{F}^{n}\right]}{\partial t}+\left[\phi_{r}-\phi_{r}^{n-1}\right]\left[\left(c_{r}+c_{w}\right) s_{F}^{n} \frac{\partial p_{F}^{n}}{\partial t}+\left[1+c_{r}\left[p_{F}^{n}-p_{r}\right]\right] \frac{\partial s_{F}^{n}}{\partial t}\right] \\
& +\nabla \cdot\left[f_{w, F}^{n}\left[\mathbf{u}-\mathbf{u}_{F}^{n}\right]+\left[f_{w}-f_{w, F}^{n}\right] \mathbf{u}_{F}^{n}\right]-\sum_{l=1}^{N_{p}}\left[f_{w, F}^{n}\left[q-q_{F}^{n}\right]+\left[f_{w}-f_{w, F}^{n}\right] q_{F}^{n}\right] \delta\left(\mathbf{x}-\mathbf{x}_{p}^{l}\right), \\
& D_{\mathcal{P}} H\left(\mathcal{P}^{n-1}, \mathcal{S}_{F}^{n}\right)\left[\mathcal{P}^{n}-\mathcal{P}^{n-1}\right]+D_{s} H\left(\mathcal{P}^{n-1}, \mathcal{S}_{F}^{n}\right)\left[\mathcal{S}-\mathcal{S}_{F}^{n}\right] \\
& =\left[\mathbf{u}-\mathbf{u}_{F}^{n}\right]+D_{s} \lambda_{F}^{n}\left[s-s_{F}^{n}\right] e^{Y^{n-1}} \nabla p_{F}^{n}+\left[Y-Y^{n-1}\right] e^{Y^{n-1}} \lambda_{F}^{n} \nabla p_{F}^{n} \\
& -\sum_{\alpha} D_{\alpha} \lambda_{F}^{n}\left[\theta_{\alpha}-\theta_{\alpha}^{n-1}\right] e^{Y^{n-1}} \nabla p_{F}^{n}+\lambda_{F}^{n} e^{Y^{n-1}} \nabla\left[p-p_{F}^{n}\right], \\
& D_{\mathcal{P}} W_{q}^{n, l}\left(\mathcal{P}^{n-1}, \mathcal{S}_{F}^{n}\right)\left[\mathcal{P}^{n}-\mathcal{P}^{n-1}\right]+D_{s} W_{q}^{n, l}\left(\mathcal{P}^{n-1}, \mathcal{S}_{F}^{n}\right)\left[\mathcal{S}-\mathcal{S}_{F}^{n}\right] \\
& =\left[q^{l}-q_{F}^{l, n}\right]-\omega_{P}^{l} D_{s} \lambda_{F}^{n} e^{Y^{n-1}}\left(P_{b h}^{l}-p_{F}^{n}\left(x_{P}^{l}\right)\left[s-s_{F}^{n}\right]\right. \\
& -\sum_{\alpha} \omega_{P}^{l} D_{\theta_{\alpha}} \lambda_{F}^{n} e^{Y^{n-1}}\left(P_{b h}^{l}-p_{F}^{n}\left(x_{P}^{l}\right)\left[\theta_{\alpha}-\theta_{\alpha}^{n-1}\right]\right. \\
& -\omega_{P}^{l} \lambda_{F}^{n} e^{Y^{n-1}}\left(P_{b h}-p_{F}^{n}\right)\left[Y-Y^{n-1}\right]+\omega_{P}^{l} \lambda_{F}^{n} e^{Y^{n-1}}\left(p-p_{F}^{n}\right), \\
& D_{\mathcal{P}} W_{Q}^{n, l}\left(\mathcal{P}^{n-1}, \mathcal{S}_{F}^{n}\right)\left[\mathcal{P}^{n}-\mathcal{P}^{n-1}\right]+D_{s} W_{Q}^{n, l}\left(\mathcal{P}^{n-1}, \mathcal{S}_{F}^{n}\right)\left[\mathcal{S}-\mathcal{S}_{F}^{n}\right] \\
& =-\omega_{I}^{i, l} D_{s} \lambda_{F}^{n} e^{Y^{n-1}}\left(p_{b h, F}^{n}-p_{F}^{n}\right)\left[s-s_{F}^{n}\right] \\
& -\sum_{\alpha} \omega_{I}^{i, l} D_{\theta_{\alpha}} \lambda_{F}^{n} e^{Y^{n-1}}\left(p_{b h, F}^{n}-p_{F}^{n}\right)\left[\theta_{\alpha}-\theta_{\alpha}^{n-1}\right] \\
& -\omega_{I}^{l} \lambda_{F}^{n} e^{Y^{n-1}}\left(p_{b h, F}^{n}-p_{F}^{n}\right)\left[Y-Y^{n-1}\right] \\
& -\omega_{I}^{l} \lambda_{F}^{n} e^{Y^{n-1}}\left(p_{b h}^{n}-p_{b h, F}^{n}\right)+\omega_{I}^{l} \lambda_{F}^{n} e^{Y^{n-1}}\left(p-p_{F}^{n}\right) .
\end{aligned}
$$

From definitions $(6.55)$ and the fact that $L \tilde{G}\left(\mathcal{P}^{n-1}, \mathcal{S}_{F}^{n}\right)=0$, we can write (B.6)-(B.9) in the following compact form

$$
\begin{array}{rr}
\mathbf{u}-\mathbf{u}_{F}^{n}= & -\lambda_{F}^{n} e^{Y^{n-1}} \nabla\left[p-p_{F}^{n}\right]+\mathcal{T}\left[s-s_{F}^{n}, Y-Y^{n-1},\left\{\theta_{\alpha}-\theta_{\alpha}^{n-1}\right\}_{\alpha}\right] \mathbf{u}_{F}^{n}, \\
q^{l}-q_{F}^{l, n}= & -\omega_{I}^{l} \lambda_{F}^{n} e^{Y^{n-1}}\left(p-p_{F}^{n}\right)+\mathcal{T}\left[s-s_{F}^{n}, Y-Y^{n-1},\left\{\theta_{\alpha}-\theta_{\alpha}^{n-1}\right\}_{\alpha}\right] q_{F}^{n}, \\
p_{b h}^{l}-p_{b h, F}^{l, n}= & p-p_{F}^{n}-\mathcal{T}\left[s-s_{F}^{n}, Y-Y^{n-1},\left\{\theta_{\alpha}-\theta_{\alpha}^{n-1}\right\}_{\alpha}\right]\left[p_{b h}^{l, n}-p_{F}^{n}\right] .
\end{array}
$$


In addition, some simple computations show that

$$
f_{w}-f_{w, F}^{n}=\mathcal{B}\left[s-s_{F}^{n},\left\{\theta_{\alpha}-\theta_{\alpha}^{n-1}\right\}_{\alpha}\right]
$$

for $\mathcal{B}$ defined in $(6.71)$

$$
\mathcal{B}\left[s, \theta_{w}, \theta_{o}\right]=\left[\kappa_{w, s}^{n}-\kappa_{s}^{n} f_{w, F}^{n}\right] s+\left[\kappa_{w}^{n}-\kappa_{w}^{n} f_{w, F}^{n}\right] \theta_{w}-\kappa_{o}^{n} f_{w, F}^{n} \theta_{o} .
$$

Now observe that

$$
\begin{array}{r}
f_{w, F}^{n}\left[\mathbf{u}-\mathbf{u}_{F}^{n}\right]+\left[f_{w}-f_{w, F}^{n}\right] \mathbf{u}_{F}^{n} \\
=-\lambda_{w, F}^{n} e^{Y^{n-1}} \nabla\left[p-p_{F}^{n}\right]+f_{w} \mathcal{T}\left[s-s_{F}^{n}, Y-Y^{n-1},\left\{\theta_{\alpha}-\theta_{\alpha}^{n-1}\right\}_{\alpha}\right] \mathbf{u}_{F}^{n} \\
+\left[\kappa_{w, s}^{n}-\kappa_{s}^{n} f_{w, F}^{n}\right]\left[s-s_{F}^{n}\right]+\left[\kappa_{w}^{n}-\kappa_{w}^{n} f_{w, F}^{n}\right]\left[\theta_{w}-\theta_{w}^{n-1}\right]-\kappa_{o}^{n} f_{w, F}^{n}\left[\theta_{o}-\theta_{o}^{n-1}\right] \mathbf{u}_{F}^{n}
\end{array}
$$

and some algebraic manipulation yields

$$
\begin{array}{r}
f_{w, F}^{n}\left[\mathbf{u}-\mathbf{u}_{F}^{n}\right]+\left[f_{w}-f_{w, F}^{n}\right] \mathbf{u}_{F}^{n} \\
=\left[\kappa_{w, s}^{n}\left[s-s_{F}^{n}\right]+\kappa_{w}^{n}\left[\theta_{w}-\theta_{w}^{n-1}\right]+f_{w, F}^{n}\left[Y-Y^{n-1}\right]\right] \mathbf{u}_{F}^{n} \\
-\lambda_{w, F}^{n} e^{Y^{n-1}} \nabla\left[p-p_{F}^{n}\right]=\mathcal{T}_{w}\left[s-s_{F}^{n}, Y-Y^{n-1},\left\{\theta_{\alpha}-\theta_{\alpha}^{n-1}\right\}_{\alpha}\right] \mathbf{u}_{F}^{n} .
\end{array}
$$

Similarly

$$
\begin{array}{r}
f_{w, F}^{n}\left[q-q_{F}^{n}\right]+\left[f_{w}-f_{w, F}^{n}\right] q_{F}^{n} \\
=\left[\kappa_{w, s}^{n}\left[s-s_{F}^{n}\right]+\kappa_{w}^{n}\left[\theta_{w}-\theta_{w}^{n-1}\right]+f_{w, F}^{n}\left[Y-Y^{n-1}\right]\right] q_{F}^{n} \\
-\omega_{I}^{p, l} \lambda_{w, F}^{n} e^{Y^{n-1}}\left(p-p_{F}^{n}\right)=\mathcal{T}_{w}\left[s-s_{F}^{n}, Y-Y^{n-1}, \theta_{w}-\theta_{w}^{n-1}\right] q_{F}^{n} .
\end{array}
$$

Equation (B.15) and (B.16) are substituted in (B.4)-(B.5) to obtain

$$
\begin{array}{r}
D_{\mathcal{P}} G\left(\mathcal{P}^{n-1}, \mathcal{S}_{F}^{n}\right)\left[\mathcal{P}^{n}-\mathcal{P}^{n-1}\right]+D_{s} G\left(\mathcal{P}^{n-1}, \mathcal{S}_{F}^{n}\right)\left[\mathcal{S}-\mathcal{S}_{F}^{n}\right] \\
=\phi_{F}^{n} c\left(s_{F}^{n}\right) \frac{\partial\left[p-p_{F}^{n}\right]}{\partial t}+\phi_{F}^{n}\left[c_{w}-c_{o}\right] \frac{\partial p_{F}^{n}}{\partial t}\left[s-s_{F}^{n}\right]+\left[\phi_{r}-\phi_{r}^{n-1}\right] c\left(s_{F}^{n}\right) \frac{\partial p_{F}^{n}}{\partial t} \\
+\nabla \cdot\left[\mathcal{T}\left[s-s_{F}^{n}, Y-Y^{n-1},\left\{\theta_{\alpha}-\theta_{\alpha}^{n-1}\right\}_{\alpha}\right] \mathbf{u}_{F}^{n}-\lambda_{F}^{n} e^{Y^{n-1}} \nabla\left[p-p_{F}^{n}\right]\right] \\
-\sum_{l=1}^{N_{p}}\left[\mathcal{T}\left[s-s_{F}^{n}, Y-Y^{n-1},\left\{\theta_{\alpha}-\theta_{\alpha}^{n-1}\right\}_{\alpha}\right] q_{F}^{n}-\omega_{I}^{p, l} \lambda_{F}^{n} e^{Y^{n-1}}\left(p-p_{F}^{n}\right)\right] \delta\left(\mathbf{x}-\mathbf{x}_{p}^{l}\right)
\end{array}
$$


and

$$
\begin{array}{r}
D_{\mathcal{P}} G_{w}\left(\mathcal{P}^{n-1}, \mathcal{S}_{F}^{n}\right)\left[\mathcal{P}^{n}-\mathcal{P}^{n-1}\right]+D_{s} G_{w}\left(\mathcal{P}^{n-1}, \mathcal{S}_{F}^{n}\right)\left[\mathcal{S}-\mathcal{S}_{F}^{n}\right] \\
=\phi_{F}^{n}\left(c_{r}+c_{w}\right) s_{F}^{n} \frac{\partial\left[p-p_{F}^{n}\right]}{\partial t}+c_{r} \phi_{r}^{n-1}\left[p-p_{F}^{n}\right] \frac{\partial s_{F}^{n}}{\partial t}+\phi_{F}^{n}\left(c_{r}+c_{w}\right)\left[s-s_{F}^{n}\right] \frac{\partial p_{F}^{n}}{\partial t} \\
+\phi_{F}^{n} \frac{\partial\left[s-s_{F}^{n}\right]}{\partial t}+\left[\phi_{r}-\phi_{r}^{n-1}\right]\left[\left(c_{r}+c_{w}\right) s_{F}^{n} \frac{\partial p_{F}^{n}}{\partial t}+\left[1+c_{r}\left[p_{F}^{n}-p_{r}\right]\right] \frac{\partial s_{F}^{n}}{\partial t}\right] \\
+\nabla \cdot\left[\mathcal{T}_{w}\left[s-s_{F}^{n}, Y-Y^{n-1}, \theta_{w}-\theta_{w}^{n-1}\right] \mathbf{u}_{F}^{n}-\lambda_{w, F}^{n} e^{Y^{n-1}} \nabla\left[p-p_{F}^{n}\right]\right] \\
-\sum_{l=1}^{N_{p}}\left[\mathcal{T}_{w}\left[s-s_{F}^{n}, Y-Y^{n-1}, \theta_{w}-\theta_{w}^{n-1}\right] q_{F}^{n}-\omega_{I}^{p, l} \lambda_{w, F}^{n} e^{Y^{n-1}}\left(p-p_{F}^{n}\right)\right] \delta\left(\mathbf{x}-\mathbf{x}_{p}^{l}\right) .
\end{array}
$$

which shows (6.51)-(6.52). Similar argument yields (6.53) and (6.55)

\section{B.1.1 Measurement Functional on the linearized variables}

In order to compute the representer coefficients, we need to study the measurement functional on the linearized variables. From (B.9)-(B.12) we observe that

$$
\begin{array}{r}
\mathcal{L}_{I}^{l, m}\left(p_{b h}^{l}\right)=\int_{0}^{T} \delta\left(t-t_{I}^{l, m}\right) p_{b h}^{l}(t)=p_{b h, F}^{l, n}\left(t_{I}^{l, m}\right) \\
\left.-\int_{0}^{T} \delta\left(t-t_{I}^{l, m}\right)\left[\left[p_{b h}^{l, n}-p_{F}^{n}\right] \mathcal{T}\left[s-s_{F}^{n}, Y-Y^{n-1},\left\{\theta_{\alpha}-\theta_{\alpha}^{n-1}\right\}\right]\right]-\left[p-p_{F}^{n}\right]\right] \\
\mathcal{L}_{p}^{l, m}\left(q^{l}(t), f_{w}\left(x_{p}^{l}, t\right)\right)=\int_{0}^{T} \delta\left(t-t_{p}^{l, m}\right)\left[a q^{l}(t)+b f_{w}\left(x_{p}^{l}, t\right)\right] \\
=a q_{F}^{l, n}\left(t_{p}^{l, m}\right)+b f_{w, F}^{n}\left(x_{p}^{l}, t_{p}^{l, m}\right) \\
+\int_{0}^{T} \delta\left(t-t_{p}^{l, m}\right)\left[a q\left[q_{F}^{n} \mathcal{T}\left[s-s_{F}^{n}, Y-Y^{n-1},\left\{\theta_{\alpha}-\theta_{\alpha}^{n-1}\right\}\right]-\omega_{I}^{p, l} \lambda_{F}^{n} e^{Y^{n-1}}\left(p-p_{F}^{n}\right)\right]\right. \\
\left.+b \mathcal{B}\left[s-s_{F}^{n},\left\{\theta_{\alpha}-\theta_{\alpha}^{n-1}\right\}\right]\right]
\end{array}
$$

and, by definition (6.39),

$$
\mathcal{L}_{o}^{l, m}(p)=\int_{0}^{T} \delta\left(t-t_{o}^{l, m}\right) p\left(x_{o}^{l}, t\right)=p\left(x_{o}^{l}, t_{o}^{l, m}\right)
$$




\section{B.1.2 The EL equations}

For the derivation of the EL equations we first observe that

$$
\begin{array}{r}
2 D_{p} J=D_{p} J_{d}+\int_{\Omega} \int_{0}^{T}\left[\Lambda\left[\phi_{F}^{n} c\left(s_{F}^{n}\right) \frac{\partial \hat{p}}{\partial t}-\nabla \cdot\left[\lambda_{F}^{n} e^{Y^{n-1}} \nabla \hat{p}\right]+\sum_{l=1}^{N_{p}} \omega_{I}^{p, l} \lambda_{F}^{n} e^{Y^{n-1}} \hat{p} \delta\left(\mathbf{x}-\mathbf{x}_{p}^{l}\right)\right]\right. \\
\left.+\Lambda_{w}\left[\phi_{F}^{n}\left(c_{r}+c_{w}\right) s_{F}^{n} \frac{\partial \hat{p}}{\partial t}+c_{r} \phi_{r}^{n-1} \hat{p} \frac{\partial s_{F}^{n}}{\partial t}-\nabla \cdot\left[\lambda_{w, F}^{n} e^{Y^{n-1}} \nabla \hat{p}\right]+\sum_{l=1}^{N_{p}} \omega_{p}^{l} \lambda_{w, F}^{n} e^{Y^{n-1}} \hat{p} \delta\left(\mathbf{x}-\mathbf{x}_{p}^{l}\right)\right]\right] \\
+\int_{\partial \Omega} \int_{0}^{T}\left[\Lambda \lambda_{F}^{n} e^{Y^{n-1}} \nabla \hat{p} \cdot \mathbf{n}+\Lambda_{w} D \lambda_{w, F}^{n} e^{Y^{n-1}} \nabla \hat{p} \cdot \mathbf{n}\right]+\int_{\Omega}\left[\Lambda \phi_{F}^{n} c\left(s_{F}^{n}\right)+\Lambda_{w} \phi_{F}^{n}\left(c_{r}+c_{w}\right) s_{F}^{n}\right] \hat{p}(t=0) .
\end{array}
$$

We integrate by parts once with respect time and twice with respect $x$. Then we assume $\Lambda=0=\Lambda_{w}$ on $t=T$ and

$$
\lambda_{F}^{n} e^{Y^{n-1}} \nabla \Lambda+\lambda_{w, F}^{n} e^{Y^{n-1}} \nabla \Lambda_{w}=0
$$

to find

$$
\begin{array}{r}
2 D_{p} J=D_{p} J_{d}+\int_{\Omega} \int_{0}^{T}\left[-\frac{\partial}{\partial t}\left[\Lambda \phi_{F}^{n} c\left(s_{F}^{n}\right)\right] \hat{p}-\frac{\partial}{\partial t}\left[\Lambda_{w} \phi_{F}^{n}\left(c_{r}+c_{w}\right) s_{F}^{n}\right]+\Lambda_{w} c_{r} \phi_{r}^{n-1} \frac{\partial s_{F}^{n}}{\partial t}\right] \hat{p} \\
-\int_{\Omega} \int_{0}^{T} \nabla \cdot\left[\lambda_{F}^{n} e^{Y^{n-1}} \nabla \Lambda+\lambda_{w, F}^{n} e^{Y^{n-1}} \nabla \Lambda_{w}\right] \hat{p} \\
+\sum_{l=1}^{N_{p}} \omega_{I}^{p, l} \int_{\Omega} \int_{0}^{T}\left[\lambda_{F}^{n} e^{Y^{n-1}} \Lambda+\lambda_{w, F}^{n} e^{Y^{n-1}} \Lambda_{w}\right] \hat{p} \delta\left(\mathbf{x}-\mathbf{x}_{p}^{l}\right) .
\end{array}
$$

From 6.46) and (B.19)-(B.21) we find

$$
\begin{aligned}
D_{p} J_{d}=\sum_{l=1}^{N_{p}} \sum_{j, m}^{M_{p}^{l}} & {\left[d_{p}^{l, m}-\mathcal{L}_{p}^{l, m}\left(q^{l}\right)\right]\left[C_{p, l}^{-1}\right]_{j, m}\left[\int_{0}^{T} \int_{\Omega} \omega_{p}^{l} \lambda_{F}^{n} e^{Y^{n-1}} \hat{p} \delta\left(x-x_{p}^{l}\right) \delta\left(t-t_{p}^{l, m}\right)\right] } \\
& -\sum_{l=1}^{N_{I}} \sum_{j, m}^{M_{I}^{l}}\left[d_{I}^{l, m}-\mathcal{L}_{I}^{l, m}\left(p_{b h}^{l}\right)\right]\left[C_{I, l}^{-1}\right]_{j, m}\left[\int_{0}^{T} \int_{\Omega} \hat{p} \delta\left(x-x_{I}^{l}\right) \delta\left(t-t_{I}^{l, m}\right)\right] \\
& -\sum_{l=1}^{N_{o}} \sum_{j, m=1}^{M_{o}^{l}}\left[d_{o}^{l, m}-\mathcal{L}_{o}^{l, m}(p)\right]^{T}\left[\mathbf{C}_{o, l}^{-1}\right]_{m, j} \int_{0}^{T} \int_{\Omega} \hat{p} \delta\left(x-x_{o}^{l, m}\right) \delta\left(t-t_{o}^{l, m}\right) .
\end{aligned}
$$


Then, (B.25) becomes

$$
\begin{array}{r}
2 D_{p} J=\sum_{l=1}^{N_{p}} \sum_{j, m}^{M_{p}^{l}}\left[d_{p}^{l, m}-\mathcal{L}_{p}^{l, m}\left(q^{l}\right)\right]\left[C_{p, l}^{-1}\right]_{j, m}\left[\int_{0}^{T} \int_{\Omega} \omega_{p}^{l} \lambda_{F}^{n} e^{Y^{n-1}} \hat{p} \delta\left(x-x_{p}^{l}\right) \delta\left(t-t_{p}^{l, m}\right)\right] \\
-\sum_{l=1}^{N_{I}} \sum_{j, m}^{M_{I}^{l}}\left[d_{I}^{l, m}-\mathcal{L}_{I}^{l, m}\left(p_{b h}^{l}\right)\right]\left[C_{I, l}^{-1}\right]_{j, m}\left[\int_{0}^{T} \int_{\Omega} \hat{p} \delta\left(x-x_{I}^{l}\right) \delta\left(t-t_{I}^{l, m}\right)\right] \\
-\sum_{\Omega=1}^{N_{o}} \sum_{j, m=1}^{M_{o}^{l}}\left[d_{o}^{l, m}-\mathcal{L}_{o}^{l, m}(p)\right]^{T}\left[\mathbf{C}_{o, l}^{-1}\right]_{m, j} \int_{0}^{T} \int_{\Omega} \hat{p} \delta\left(x-x_{o}^{l, m}\right) \delta\left(t-t_{o}^{l, m}\right) \\
\left.\quad-\frac{\partial}{\partial t}\left[\Lambda \phi_{F}^{n} c\left(s_{F}^{n}\right)\right] \hat{p}-\frac{\partial}{\partial t}\left[\Lambda_{w} \phi_{F}^{n}\left(c_{r}+c_{w}\right) s_{F}^{n}\right]+\Lambda_{w} c_{r} \phi_{r}^{n-1} \frac{\partial s_{F}^{n}}{\partial t}\right] \hat{p} \\
\quad \int_{\Omega}^{T} \int_{0}^{T} \nabla \cdot\left[\lambda_{F}^{n} e^{Y^{n-1}} \nabla \Lambda+\lambda_{w, F}^{n} e^{Y^{n-1}} \nabla \Lambda_{w}\right] \hat{p} \\
\sum_{l=1}^{N_{p}} \omega_{p}^{l} \int_{\Omega} \int_{0}^{T}\left[\lambda_{F}^{n} e^{Y^{n-1}} \Lambda+\lambda_{w, F}^{n} e^{Y^{n-1}} \Lambda_{w}\right] \hat{p} \delta\left(x-x_{p}^{l}\right),
\end{array}
$$

which in turn yields

$$
\begin{array}{r}
\mathcal{A}^{n}\left[\Lambda, \Lambda_{w}\right]=\sum_{l=1}^{N_{I}} \sum_{j, m}^{M_{I}^{l}}\left[d_{I}^{l, m}-\mathcal{L}_{I}^{l, m}\left(p_{b h}^{l}\right)\right]\left[C_{I, l}^{-1}\right]_{j, m}\left[\int_{0}^{T} \int_{\Omega} \hat{p} \delta\left(x-x_{I}^{l}\right) \delta\left(t-t_{I}^{l, m}\right)\right] \\
-\sum_{l=1}^{N_{p}} \sum_{j, m}^{M_{p}^{l}}\left[d_{p}^{l, m}-\mathcal{L}_{p}^{l, m}\left(q^{l}\right)\right]\left[C_{p, l}^{-1}\right]_{j, m}\left[\int_{0}^{T} \int_{\Omega} \omega_{p}^{l} \lambda_{F}^{n} e^{Y^{n-1}} \hat{p} \delta\left(x-x_{p}^{l}\right) \delta\left(t-t_{p}^{l, m}\right)\right] \\
+\sum_{l=1}^{N_{o}} \sum_{j, m=1}^{M_{o}^{l}}\left[d_{o}^{l, m}-\mathcal{L}_{o}^{l, m}(p)\right]^{T}\left[\mathbf{C}_{o, l}^{-1}\right]_{m, j} \int_{0}^{T} \int_{\Omega} \hat{p} \delta\left(x-x_{o}^{l, m}\right) \delta\left(t-t_{o}^{l, m}\right)
\end{array}
$$


for $\mathcal{A}^{n}$ defined in (6.64). Similarly,

$$
\begin{array}{r}
2 D_{s} J=D_{s} J_{d}+\int_{\Omega} \int_{0}^{T} \Lambda\left[\phi_{F}^{n}\left[c_{w}-c_{o}\right] \frac{\partial p_{F}^{n}}{\partial t} \hat{s}+\nabla \cdot\left[\kappa_{s}^{n} \hat{s} \mathbf{u}_{F}^{n}\right]-\sum_{l=1}^{N_{p}} q_{F}^{n} \kappa_{s}^{n} \hat{s} \delta\left(x-x_{p}^{l}\right)\right] \\
+\int_{\Omega} \int_{0}^{T} \Lambda_{w}\left[\phi_{F}^{n}\left(c_{r}+c_{w}\right) \hat{s} \frac{\partial p_{F}^{n}}{\partial t}+\phi_{F}^{n} \frac{\partial \hat{s}}{\partial t}+\nabla \cdot\left[\kappa_{w, s}^{n} \hat{s} \mathbf{u}_{F}^{n}\right]-\sum_{l=1}^{N_{p}} q_{F}^{n} \tau_{w, s}^{n} \hat{s} \delta\left(x-x_{p}^{l}\right)\right] \\
\left.-\int_{\Gamma} \int_{0}^{T}\left[\Lambda\left[\kappa_{s}^{n} \hat{s} \mathbf{u}_{F}^{n} \cdot \mathbf{n}\right]+\Lambda_{w}\left[\kappa_{w, s}^{n} \hat{s} \mathbf{u}_{F}^{n} \cdot \mathbf{n}\right]\right]+\int_{\Omega}\left[\phi_{F}^{n} \hat{s}\right]\right](t=0) .
\end{array}
$$

Then, integrating by parts and using the same final time conditions on $\Lambda, \Lambda_{w}$, as well as (B.23) we obtain

$$
\begin{array}{r}
2 D_{s} J=D_{s} J_{d}+\int_{\Omega} \int_{0}^{T}\left[\Lambda \phi_{F}^{n}\left[c_{w}-c_{o}\right] \frac{\partial p_{F}^{n}}{\partial t}+\Lambda_{w} \phi_{F}^{n}\left(c_{r}+c_{w}\right) \frac{\partial p_{F}^{n}}{\partial t}-\frac{\partial\left[\phi_{F}^{n} \Lambda_{w}\right]}{\partial t}\right] \hat{s} \\
-\int_{\Omega} \int_{0}^{T}\left[\nabla \Lambda \cdot\left[\kappa_{s}^{n} \hat{s} \mathbf{u}_{F}^{n}\right]+\nabla \Lambda_{w} \cdot\left[\kappa_{w, s}^{n} \hat{s} \mathbf{u}_{F}^{n}\right]\right]-\sum_{l=1}^{N_{p}} \int_{\Omega} \int_{0}^{T} q_{F}^{n}\left[\Lambda \kappa_{s}^{n}+\Lambda_{w} \kappa_{w, s}^{n}\right] \hat{s} \delta\left(x-x_{p}^{l}\right)
\end{array}
$$

On the other hand, is easy to see that

$$
\begin{gathered}
\int_{\Omega} \int_{0}^{T}\left[\kappa_{s}^{n} \nabla \Lambda \cdot \mathbf{u}_{F}^{n}+\kappa_{w, s}^{n} \nabla \Lambda_{w} \mathbf{u}_{F}^{n}\right] \hat{s} \\
=\int_{\Omega} \int_{0}^{T}\left[\nabla \cdot\left[\Lambda \kappa_{s}^{n} \mathbf{u}_{F}^{n}\right]+\nabla \cdot\left[\Lambda_{w} \kappa_{w, s}^{n} \mathbf{u}_{F}^{n}\right]\right] \hat{s}-\int_{\Omega} \int_{0}^{T}\left[\Lambda \nabla \cdot\left[\kappa_{s}^{n} \mathbf{u}_{F}^{n}\right]+\Lambda \nabla \cdot\left[\kappa_{s}^{n} \mathbf{u}_{F}^{n}\right]\right] \hat{s}(\mathrm{~B} .30)
\end{gathered}
$$

Therefore,

$$
\begin{array}{r}
2 D_{s} J=D_{s} J_{d}+\int_{\Omega} \int_{0}^{T}\left[\Lambda \phi_{F}^{n}\left[c_{w}-c_{o}\right] \frac{\partial p_{F}^{n}}{\partial t}+\Lambda_{w} \phi_{F}^{n}\left(c_{r}+c_{w}\right) \frac{\partial p_{F}^{n}}{\partial t}-\frac{\partial\left[\phi_{F}^{n} \Lambda_{w}\right]}{\partial t}\right] \hat{s} \\
-\int_{\Omega} \int_{0}^{T}\left[\nabla \cdot\left[\Lambda \kappa_{s}^{n} \mathbf{u}_{F}^{n}\right]+\nabla \cdot\left[\Lambda_{w} \kappa_{w, s}^{n} \mathbf{u}_{F}^{n}\right]\right] \hat{s}+\int_{\Omega} \int_{0}^{T}\left[\Lambda \nabla \cdot\left[\kappa_{s}^{n} \mathbf{u}_{F}^{n}\right]+\Lambda \nabla \cdot\left[\kappa_{s}^{n} \mathbf{u}_{F}^{n}\right]\right] \hat{s} \\
-\sum_{l=1}^{N_{p}} \int_{\Omega} \int_{0}^{T} q_{F}^{n}\left[\Lambda \kappa_{s}^{n}+\Lambda_{w} \kappa_{w, s}^{n}\right] \hat{s} \delta\left(x-x_{p}^{l}\right) .
\end{array}
$$


From 6.46) and (B.19)-(B.21) we compute

$$
\begin{array}{r}
D_{s} J_{d}=-\sum_{l=1}^{N_{p}} \sum_{j m}^{M_{p}^{l}}\left[d_{p}^{l, j}-\mathcal{L}_{p}^{l, j}\left(q^{l}(t), f_{w}\left(x_{p}^{l}, t\right)\right)\right]\left[C_{p, l}^{-1}\right]_{j, m}\left[\int _ { 0 } ^ { T } \int _ { \Omega } \left[a q_{F}^{n} \kappa_{s}^{n}\right.\right. \\
\left.\left.-b\left[\kappa_{w, s}^{n}-\kappa_{s}^{n} f_{w, F}^{n}\right]\right] \delta\left(x-x_{p}^{l}\right) \delta\left(t-t_{p}^{l, m}\right)\right] \\
+\sum_{l=1}^{N_{I}} \sum_{j, m}^{M_{I}^{l}}\left[d_{I}^{l, j}-\mathcal{L}_{I}^{l, j}\left(p_{b h}^{l}\right)\right]\left[C_{I, l}^{-1}\right]_{j, m}\left[\int_{0}^{T} \int_{\Omega}\left[p_{b h, F}^{l, n}-p_{F}^{n}\right] \kappa_{s}^{n} \hat{s} \delta\left(t-t_{I}^{l, m}\right) \delta\left(x-x_{I}^{l}\right)\right]
\end{array}
$$

and so (B.31) can be written as

$$
\begin{array}{r}
2 D_{s} J=-\sum_{l=1}^{N_{p}} \sum_{j m}^{M_{p}^{l}}\left[d_{p}^{l, j}-\mathcal{L}_{p}^{l, j}\left(q^{l}(t), f_{w}\left(x_{p}^{l}, t\right)\right)\right]\left[C_{p, l}^{-1}\right]_{j, m}\left[\int _ { 0 } ^ { T } \int _ { \Omega } \left[a q_{F}^{n} \kappa_{s}^{n}\right.\right. \\
\left.\left.-b\left[\kappa_{w, s}^{n}-\kappa_{s}^{n} f_{w, F}^{n}\right]\right] \delta\left(x-x_{p}^{l}\right) \delta\left(t-t_{p}^{l, m}\right)\right] \\
+\sum_{l=1}^{N_{I}} \sum_{j, m}^{M_{I}^{l}}\left[d_{I}^{l, j}-\mathcal{L}_{I}^{l, j}\left(p_{b h}^{l}\right)\right]\left[C_{I, l}^{-1}\right]_{j, m}\left[\int_{0}^{T} \int_{\Omega}\left[p_{b h, F}^{l, n}-p_{F}^{n}\right] \kappa_{s}^{n} \hat{s} \delta\left(t-t_{I}^{l, m}\right) \delta\left(x-x_{I}^{l}\right)\right] \\
+\int_{\Omega} \int_{0}^{T}\left[\Lambda \phi_{F}^{n}\left[c_{w}-c_{o}\right] \frac{\partial p_{F}^{n}}{\partial t}+\Lambda_{w} \phi_{F}^{n}\left(c_{r}+c_{w}\right) \frac{\partial p_{F}^{n}}{\partial t}-\frac{\partial\left[\phi_{F}^{n} \Lambda_{w}\right]}{\partial t}\right] \hat{s} \\
-\int_{\Omega} \int_{0}^{T}\left[\nabla \cdot\left[\Lambda \kappa_{s}^{n} \mathbf{u}_{F}^{n}\right]+\nabla \cdot\left[\Lambda_{w} \kappa_{w, s}^{n} \mathbf{u}_{F}^{n}\right]\right] \hat{s}+\int_{\Omega} \int_{0}^{T}\left[\Lambda \nabla \cdot\left[\kappa_{s}^{n} \mathbf{u}_{F}^{n}\right]+\Lambda \nabla \cdot\left[\kappa_{s}^{n} \mathbf{u}_{F}^{n}\right]\right] \hat{s} \\
\left.-\sum_{l=1}^{N_{p}} \int_{\Omega} \int_{0}^{T} q_{F}^{n}\left[\Lambda q_{F}^{n} \tau_{s}^{n}+\Lambda_{w} \tau_{w, s}^{n}\right] \hat{s} \delta\left(\mathbf{x}-\mathbf{x}_{p}^{l}\right)\right],
\end{array}
$$

which is equivalent to

$$
\begin{array}{r}
\mathcal{A}_{w}^{n}\left[\Lambda, \Lambda_{w}\right]=\sum_{l=1}^{N_{p}} \sum_{j m}^{M_{p}^{l}}\left[d_{p}^{l, j}-\mathcal{L}_{p}^{l, j}\left(q^{l}(t), f_{w}\left(x_{p}^{l}, t\right)\right)\right]\left[C_{p, l}^{-1}\right]_{j, m}\left[\int _ { 0 } ^ { T } \int _ { \Omega } \left[a q_{F}^{n} \kappa_{s}^{n}\right.\right. \\
\left.\left.-b\left[\kappa_{w, s}^{n}-\kappa_{s}^{n} f_{w, F}^{n}\right]\right] \delta\left(x-x_{p}^{l}\right) \delta\left(t-t_{p}^{l, m}\right)\right] \\
-\sum_{l=1}^{N_{I}} \sum_{j, m}^{M_{I}^{l}}\left[d_{I}^{l, j}-\mathcal{L}_{I}^{l, j}\left(p_{b h}^{l}\right)\right]\left[C_{I, l}^{-1}\right]_{j, m}\left[\int_{0}^{T} \int_{\Omega}\left[p_{b h, F}^{l, n}-p_{F}^{n}\right] \kappa_{s}^{n} \hat{s} \delta\left(t-t_{I}^{l, m}\right) \delta\left(x-x_{I}^{l}\right)\right]
\end{array}
$$


for $\mathcal{A}_{w}^{n}$ defined in (6.65). Analogously,

$$
\begin{array}{r}
2 D_{Y} J=D_{Y} J_{d}+\int_{\Omega} \int_{\Omega}\left(Y-Y^{n-1}\right) C_{Y}^{-1}(x, y) \hat{Y} \\
+\int_{\Omega} \int_{0}^{T} \Lambda\left[\nabla \cdot\left[\lambda_{F}^{n} \hat{Y} \mathbf{u}_{F}^{n}\right]-\sum_{l=1}^{N_{p}} q_{F}^{n} \hat{Y} \delta\left(x-x_{p}^{l}\right)\right] \\
+\Lambda_{w}\left[\nabla \cdot\left[\lambda_{w, F}^{n} \hat{Y} \mathbf{u}_{F}^{n}\right]-\sum_{l=1}^{N_{p}} f_{w, F}^{n} q_{F}^{n} \hat{Y} \delta\left(x-x_{p}^{l}\right)\right] \\
-\int_{\Gamma} \int_{0}^{T}\left[\Lambda \lambda_{F}^{n} \hat{Y} \mathbf{u}_{F}^{n} \cdot \mathbf{n}+\Lambda_{w, F}^{n} \hat{Y} \mathbf{u}_{F}^{n} \cdot \mathbf{n}\right]
\end{array}
$$

and the same arguments as before show

$$
\begin{array}{r}
Y-Y^{n-1}=\int_{\Omega} \int_{0}^{T} C_{Y}^{-1}(x, y)[ \\
{\left[\nabla \Lambda+f_{w, F}^{n} \nabla \Lambda_{w}\right] \cdot \mathbf{u}_{F}^{n}+\sum_{l=1}^{N_{p}} q_{F}^{n}\left[\Lambda+f_{w, F}^{n} \Lambda_{w}\right] \delta\left(x-x_{p}^{l}\right)} \\
+\sum_{l=1}^{N_{p}} \sum_{j m}^{M_{p}^{l}}\left[d_{q}^{j, l}-\mathcal{L}_{q}^{j, l}\left(q^{l}\right)\right]\left[C_{p, l}^{-1}\right]_{j, m}\left[q_{F}^{n} \delta\left(x-x_{p}^{l}\right) \delta\left(t-t_{p}^{l, m}\right)\right] \\
\left.\left.-\sum_{l=1}^{N_{I}} \sum_{j, m}^{M_{I}^{l}}\left[d_{I}^{l, j}-\mathcal{L}_{I}^{l, j}\left(p_{b h}^{l}\right)\right]\left[C_{I, l}^{-1}\right]_{j, m}\left[p_{b h, F}^{l, n}-p_{F}^{n}\right] \delta\left(t-t_{I}^{l, m}\right) \delta\left(x-x_{I}^{l}\right)\right]\right] .
\end{array}
$$

Expressions (6.67)-(6.69) are obtained with the same arguments.

\section{B.2 The Representers Algorithm}

Note that $\mathcal{A}$ and $\mathcal{A}_{w}$ (6.64)-(6.65) are bilinear operators. Then, expressions (6.83)-(6.84) are substituted in (6.62)-(6.63) we find that

$$
\sum_{l=1}^{N_{\chi}} \sum_{j=1}^{M_{\chi}^{l}} \beta_{\chi}^{l, j} \mathcal{A}\left[\Lambda_{\chi}^{l, j}, \Lambda_{\chi, w}^{l, j}\right]=\sum_{l=1}^{N_{\chi}} \sum_{j, m=1}^{M_{\chi}^{l}}\left[d_{\chi}^{l, j}-\mathcal{L}_{\chi}^{l, j}\right]\left[C_{\chi, l}^{-1}\right]_{j, m} \mathbf{D}_{1, \chi}^{l, m}
$$


Then, $\Lambda_{\chi}^{l, j}$ and $\Lambda_{\chi, w}^{l, j}$ satisfies (6.85)-(6.86) if and only if

$$
\beta_{\chi}^{l, m}=\sum_{j=1}^{M_{\chi}^{l}}\left[d_{\chi}^{l, j}-\mathcal{L}_{\chi}^{l, j}\right]\left[C_{\chi, l}^{-1}\right]_{j, m}
$$

for every $\chi \in\{p, I, o\}, l \in\left\{1, \ldots, N_{\chi}\right\}$ and $m \in\left\{1, \ldots, M_{\chi}^{l}\right\}$. From definition (6.81), expression (B.38) becomes

$$
\beta_{\chi}^{l}=\left[\mathbf{d}_{\chi}^{l}-\mathcal{L}_{\chi}^{l}\right] \mathbf{C}_{\chi, l}^{-1}
$$

which finally can be written as

$$
\mathbf{C}_{\chi, l} \beta_{\chi}^{l}=\left[\mathbf{d}_{\chi}^{l}-\mathcal{L}_{\chi}^{l}\right]
$$

The same argument of linearity shows (6.87)-(6.90) as well as

$$
\mathcal{T}\left[s-s_{F}^{n}, Y-Y^{n-1},\left\{\theta_{\alpha}-\theta_{\alpha}^{n-1}\right\}\right]=\sum_{\chi} \sum_{l=1}^{N_{\chi}} \sum_{j=1}^{M_{\chi}^{l}} \beta_{j}^{\chi, l} \mathcal{T}\left[\mathbf{r}_{j}^{\chi, l}\right]
$$

where $\mathbf{r}_{j}^{\chi, l}$ was defined in (6.72). Therefore, it follows from (B.20) that

$$
\mathcal{L}_{p}^{k, m}\left(q^{k}\right)=\mathcal{L}_{p}^{k, m}\left(q_{F}^{k, n}\right)+\sum_{\chi} \sum_{l=1}^{N_{\chi}} \sum_{j=1}^{M_{\chi}^{l}} \beta_{\chi}^{l, j} \int_{0}^{T} \delta\left(t-t_{m}^{P, k}\right)\left[q_{F}^{n} \mathcal{T}\left[\mathbf{r}_{j}^{\chi, l}\right]-\omega_{P}^{k} \lambda_{F}^{n} e^{Y^{n-1}} R_{\chi, p}^{l, j}\right](\mathrm{B}
$$

and from definition (6.75)-(6.76) we obtain

$$
\mathcal{L}_{q}^{k, m}\left(q^{k}\right)=\int_{0}^{T} \delta\left(t-t_{m}^{P, l}\right) q^{k}(t)=\mathcal{L}_{q}^{k, m}\left(q_{F}^{k, n}\right)+\sum_{\chi} \sum_{l=1}^{N_{\chi}} \boldsymbol{\tau}_{q, \chi}^{k, m, l} \cdot \mathbf{b}_{\chi}^{l} .
$$

Similarly, from (B.21)

$$
\mathcal{L}_{I}^{k, m}\left(p_{b h}^{k}\right)=\mathcal{L}_{I}^{k, m}\left(p_{b h, F}^{k, n}\right)-\sum_{\chi} \sum_{l=1}^{N_{\chi}} \sum_{j=1}^{M_{\chi}^{l}} \beta_{\chi}^{l, j} \int_{0}^{T} \delta\left(t-t_{m}^{I, k}\right)\left[\left[p_{b h}^{l, n}-p_{F}^{n}\right] \mathcal{T}\left[\mathbf{r}_{j}^{\chi, l}\right]-R_{\chi, p}^{l, j}\right]
$$

and so

$$
\mathcal{L}_{I}^{k, m}\left(p_{b h}^{k}\right)=\mathcal{L}_{I}^{k, m}\left(p_{b h, F}^{k, n}\right)+\sum_{\chi} \sum_{l=1}^{N_{\chi}} \boldsymbol{\tau}_{I, \chi}^{k, m, l} \cdot \mathbf{b}_{\chi}^{l}
$$


Analogously,

$$
\mathcal{L}_{o}^{k, m}(p)=\mathcal{L}_{o}^{k, m}\left(p_{F}^{n}\right)+\sum_{\chi} \sum_{l=1}^{N_{\chi}} \boldsymbol{\tau}_{o, \chi}^{k, m, l} \cdot \mathbf{b}_{\chi}^{l}
$$

Then, for $\xi \in\{p, I, o\}$, the general case can be written as

$$
\mathcal{L}_{\xi}^{k, m}(\xi)=\mathcal{L}_{\xi}^{k, m}\left(\xi_{F}^{n}\right)+\sum_{\chi} \sum_{l=1}^{N_{\chi}} \boldsymbol{\tau}_{\xi, \chi}^{k, m, l} \cdot \mathbf{b}_{\chi}^{l} .
$$

On the other hand, substituting (B.47) in (B.38) yields

$$
\left[\mathbf{C}_{\xi, k} \mathbf{b}_{\xi}^{k}\right]_{m}=\mathbf{d}_{\xi}^{k, m}-\mathcal{L}_{\xi}^{k, m}\left(\xi_{F}^{n}\right)-\sum_{\chi} \sum_{l=1}^{N_{\chi}} \boldsymbol{\tau}_{\xi, \chi}^{k, m, l} \cdot \mathbf{b}_{\chi}^{l}
$$

which by using (6.77) and (6.41) becomes

$$
\mathbf{C}_{\xi, k} \mathbf{b}_{\xi}^{k}+\sum_{\chi} \sum_{l=1}^{N_{\chi}} \boldsymbol{v}_{\xi, \chi}^{k, l} \cdot \mathbf{b}_{\chi}^{l}=\mathbf{d}_{\xi}^{k}-\mathcal{L}_{\xi}^{k}\left(\xi_{F}^{n}\right)
$$

Note that (B.49) can be written as

$$
\left[\mathbf{C}_{\xi, k}+\boldsymbol{v}_{\xi, \xi}^{k, k}\right] \mathbf{b}_{\xi}^{k}+\sum_{l \neq k}^{N_{\xi}} \boldsymbol{v}_{\xi, \xi}^{k, l} \cdot \mathbf{b}_{\xi}^{l}+\sum_{\chi \neq \xi} \sum_{l=1}^{N_{\chi}} \boldsymbol{v}_{\xi, \chi}^{k, l} \cdot \mathbf{b}_{\chi}^{l}=\mathbf{d}_{\xi}^{k}-\mathcal{L}_{\xi}^{k}\left(\xi_{F}^{n}\right)
$$

From (6.78), the previous expression becomes

$$
\left[\mathbf{V}_{\chi, \chi}^{k, k}\right] \mathbf{b}_{\xi}^{k}+\sum_{l \neq k}^{N_{\xi}} \mathbf{V}_{\xi, \xi}^{k, l} \cdot \mathbf{b}_{\xi}^{l}+\sum_{\chi \neq \xi} \sum_{l=1}^{N_{\chi}} \mathbf{V}_{\xi, \chi}^{k, l} \cdot \mathbf{b}_{\chi}^{l}=\mathbf{d}_{\xi}^{k}-\mathcal{L}_{\xi}^{k}\left(\xi_{F}^{n}\right)
$$

Simple computations then show that (B.51) is

$$
\sum_{\chi} \sum_{l=1}^{N_{\chi}} \mathbf{V}_{\xi, \chi}^{k, l} \cdot \mathbf{b}_{\chi}^{l}=\mathbf{d}_{\xi}^{k}-\mathcal{L}_{\xi}^{k}\left(\xi_{F}^{n}\right)
$$

which in turn, by (6.42) and (6.80), implies

$$
\sum_{\chi} \sum_{l=1}^{N_{\chi}} \mathbf{V}_{\xi, \chi}^{l} \cdot \mathbf{b}_{\chi}^{l}=\mathbf{d}_{\xi}-\mathcal{L}_{\xi}
$$


Finally, from (6.43) we have

$$
\sum_{\chi} \mathbf{V}_{\xi, \chi} \mathbf{b}_{\chi}=\mathbf{d}_{\xi}-\mathcal{L}_{\xi}
$$

that we rewrite as

$$
\mathbf{V b}=\mathbf{d}-\mathcal{L}
$$

for $\mathbf{d}$ and $\mathcal{L}$ defined in (6.80) and $\mathbf{V}$ as described in Step (4) of Algorithm 4 


\section{Bibliography}

[1] R. A. Adams. Sobolev Spaces. Academic Press, New York, NY, 1975.

[2] T. Arbogast. The existence of weak solutions to single porosity and simple dual-porosity models of two-phase incompressible flow. J, Nonlinear Analisis: Theory, Methods and Applications, 19:1009-1031, 1992.

[3] T. Arbogast, M. F. Wheeler, and I. Yotov. Mixed finite elements for elliptic problems with tensor coefficients as cell-centered finite differences. SIAM J. Numer. Anal., 34:828-852, 1997.

[4] J. Baird and Dawson C. The representer method for two-phase flow in porous media. Compt. Geosci. DOI 10.1007/s10596-007-9048-8, 11.

[5] J. Baird and C. Dawson. The representer method for data assimilation in single-phase Darcy flow in porous media. Compt. Geosci. DOI 10.1007/s10596-005-9006-2, 9.

[6] J. Baird and C. Dawson. A posteriori error estimation of the representer method for single-phase Darcy flow. Comp. Meth. Appl. Mech. Eng., 196:1623-1632, 2007.

[7] A. Barrera. History matching by simultaneous calibration of flow functions. Ph. D. Thesis. The University of Texas at Austin, 2007.

[8] J. Bear. Dynamics of Fluids in Porous Media. Dover Pulications, New York, 1972.

[9] A. F. Bennett. Array design by inverse methods. Prog. Oceanog., 15:129-156, 1985.

[10] A. F. Bennett. Inverse Methods in Physical Oceanography. Cambridge University Press, New York, NY, 1992.

[11] A. F. Bennett. Inverse Modeling of the Ocean and Atmosphere. Cambridge University Press, Cambridge, UK, 2002.

[12] A. F. Bennett and Chua B.S. An inverse ocean modeling system. Ocean Modelling, $3: 137-165,2001$.

[13] A. F. Bennett, Chua B.S., Harrison D.E., and McPhaden M.J. Generalized inversion of Tropical Atmosphere-Ocean (TAO) data and a coupled model of the tropical Pacific. Part II: the 1995-96 La Niña and 1997-98 El Niño. Journal of Climate, 13:2770-2785, 2000 . 
[14] G. Burgers, P. J. van Leeuwen, and G. Evensen. Analysis scheme in the Ensemble Kalman Filter. Monthly Weather Review, 126(6):1719-1724, 1998.

[15] J. Caers. Petroleum Geology. Society of Petroleum Engineers, Texas, U.S.A, 1st edition, 2005.

[16] G. Chavent, M. Dupuy, and C. Lemmonier. History matching by use of optimal control. Soc. Pet. eng. J., 15:74-86, 1974.

[17] C. V. Deutsch and A. G. Journel. GSLIB: Geostatistical Software Library. Oxford University Press, New York, 2st edition, 1997.

[18] F. Le Dimet and O. Talagrand. Variational algorithms for analysis and assimilation of meteorological observations. Tellus, 38A:97-110, 1986.

[19] Y. Dong, Y. Gu, and D. S. Oliver. Sequential assimilation of 4D seismic data for reservoir description using the Ensemble Kalman Filter. J. Pet. Sci. and Eng., 53:8399, 2006.

[20] G. D. Egbert, A. F. Bennet, and M. G. G Foreman. TOPEX/POSEIDON tides estimated using a global inverse method. Journal of Geophysical Research, 99(24):812-852, 1994.

[21] H. W. Engl, M. Hanke, and A. Neubauer. Regularization of Inverse Problems. Drdrecht:Kluwer, The Netherlands, 1st edition, 1996.

[22] G. Evensen. Using the EnKF for assisted history matching of a North Sea reservoir model. Evensen G. and Hove J. and Meisingset H.C. and Reiso E. and Seim K.S.

[23] G. Evensen. Sequential data assimilation with a non-linear quasi-geostrophic model using Monte-Carlo methods to forecast error statistics. Journal of Geophysical Research, 99(C5):10143-10162, 1994.

[24] G. Evensen. The Ensemble Kalman Filter: theoretical formulation and practical implementation. Ocean Dynamics, 53:343-367, 2003.

[25] G. Evensen. Data Assimilation. Springer-Verlag, Heidelberg, 1st edition, 2007.

[26] G. Evensen and van P.J. Leeuwen. An Ensemble Smoother for nonlinear dynamics. Monthly Weather Review, 128.

[27] C. Gao and T. Rajeswaran. A literature review on smart-well technology. In procedings of SPE Production and Operations Symposium, Oklahoma City, Oklahoma.

[28] G. Gao and A. C. Reynolds. An improved implementation of the LBFGS algorithm for automatic history matching. In SPE Annual Technical Conference and Exhibition, Houston, Texas, U.S.A., 26-29 Septempber 2004, SPE 90058.

[29] G. R. Gavalas, P. C. Shah, and J. H. Seinfield. Reservoir history matching by Bayessian estimation. Soc. Pet. Eng. J.,, 16. 
[30] Y. Gu and D. S Oliver. The Ensemble Kalman Filter for continuous updating of reservoir simulation models. J. Energy Res. Tech., 128.

[31] Y. Gu and D. S Oliver. History matching of the PUNQ-S3 reservoir model using the Ensemble Kalman Filter. SPE Journal, 10.

[32] M. Hanke. A regularizing Levenberg-Marquardt scheme, with applications to inverse groundwater filtration problems. Inverse Problems, 13:79-95, 1997.

[33] http://www.spe.org/csp/. Spe 10th comparative project.

[34] S. P. Huang, S. T. Quek, and K. K. Phoon. Convergence study of the truncated Karhunen-Loeve expansion for simulation of stochastic processes. Int. J. Numer. Meth. Engng, 52:1029-1043.

[35] M. A. Iglesias and C. Dawson. An iterative representer-based schemme for data inversion in reservoir modeling. Submitted to Inverse Problems.

[36] M. A. Iglesias and C. Dawson. The respresenter method for state and parameter estimation in single-phase Darcy flow. Computer Methods in Applied Mechanics and Engineering, 196:45774596, 2007.

[37] P. Jacquard and C. Jain. Permeability distribution from field pressure data. Soc. Pet. eng. J., 5 .

[38] B. Jafarpour and D. B. McLaughlin. History matching with an Ensemble Kalman Filter and discrete cosine parameterization. Compt. Geosci.,, 12.

[39] B. Jafarpour and D. B. McLaughlin. Spe 106453: Efficient permeability parameterization with the discrete cosine transform. SPE Reservoir Simulation Symposium, 26-28 February 2007, Houston, Texas, U.S.A.

[40] R. E. Kalman. A new approach to linear filtering and prediction problems. Transactions of the ASME - Journal of Basic Engineering, 82:35-45, 1960.

[41] A. Korganoff. Sur la resulution de problemes "inverses" en hydrologie. Bu. Int. Assoc. Sci. Hydrol., 15.

[42] C. Kravaris and J. H. Seinfield. Identification of parameters in distributed parmameter system by regularization. SIAM J. Control and Opt., 23.

[43] T. Lee and J. H. Seinfield. Estimation of absolute and relative permeabilities in petroleum reservoirs. Inverse Problems, 3:711-728, 1987.

[44] R. Li, A. C. Reynolds, and D. S. Oliver D. History matching of three-phase flow production data. SPE Journal, 8.

[45] J. L. Lions and E. Magenes. Non-Homogeneous Boundary Value Problems and Applications Vol.I. Springer-Verlag, Berlin, 1972. 
[46] J. L. Lions and E. Magenes. Non-Homogeneous Boundary Value Problems and Applications Vol.II. Springer-Verlag, Berlin, 1972.

[47] N. Liu and D. S. Oliver. Ensemble Kalman Filter for automatic history matching of geologic facies. J. of Pet. Sci. and Eng., 47:147-161, 2005.

[48] R.J. Lorentzen, G. Naevdal, B. Valls, and A. M. Berg. Spe 96375: Analysis of the Ensemble Kalman Filter for estimation of permeability and porosity in reservoir models. SPE Annual Technical Conference and Exhibition, 9 - 12 October, 2005, Dallas, Texas, USA., www.spe.org.

[49] G. Naevdal, D. R. Brouwer, and J. D. Jansen. Waterflooding using closed-loop control. Compt Geosci, 10:37-60, 2006.

[50] G. Naevdal, L. M. Johnsen, S. I. Aanonsen, and E. H. Vefring. Reservoir monitoring and continuous model updating using Ensemble Kalman Filter. SPE Journal, March 2005, Published by: SPE, SPE Journal, http://www.spe.org.

[51] G. Naevdal, T. Mannseth, and E. H. Vefring. Near-well reservoir monitoring through Ensemble Kalman Filter. In SEP/DOE Improved Oil Recovery Symposium, Tulsa, Oklahoma, April 2002, SPE 75235.

[52] J. Nocedal and Stephen J. Wright. Numerical Optimization. Springe, New York, 1999.

[53] D. S. Oliver. Calculation of the inverse of the covariance. Mathematical Geology, 30 (7):911-933, 1998.

[54] D. W. Peaceman. Fundamentals of Numerical Reservoir Simulation. Elsevier Scientific Publishing Company, Amsterdam, 1977.

[55] J. K. Przybysz-Jarnut, R. G. Hanea, J. D. Jansen, and A. W. Heemink. Application of the representer method for parameter estimation in numerical reservoir models. Compt Geosci, 11:73-85, 2007.

[56] L.B. Reid. A functional inverse approach for three-dimensional characterization of subsurface contaminants. PhD thesis.

[57] A. C. Reynolds, Li R., and Oliver D. S. Simultaneous estimation of absolute and relative permeability by automatic history matching of three-phase flow production data. Journal of Canadian Petroleum Technology, 43.

[58] T. Rosmond and Xu L. Development of NAVDAS-AR: non-linear formulation and outer loop test. Tellus Series A-Dynamic Meteorology and Oceanopgraphy, 58:45-58, 2006.

[59] P. Sarma, L. J. Durlofsky, and K. Aziz. Kernel principal component analysis for efficient differentiable parameterization of multipoint geostatistics. Math. Geosci, 40, 2008. 
[60] P. Sarma, L. J. Durlofsky, and K. Aziz. Efficient closed loop optimization under uncertainty. In the proceedings of the SPE Europec/EAGE Annual Conference, Madrid, Spain, June 2005, SPE 94241.

[61] P. Sarma, L. J. Durlofsky, K. Aziz, and W. H. Chen. Efficient real-time reservoir management using adjoint-based optimal control and model updating. Compt. Geosci., 10, 2006.

[62] B. Scholkopf, A. Smola, and K-R. Muller. Nonlinear component analysis as a kernel eigenvalue problem. Neural Computations, 10, 1998.

[63] J.A. Skjervheim, Evensen G., Aanonsen S. I., Ruud B. O., and Johansen T. A. Incorporating $4 \mathrm{D}$ seismic data in reservoir simulation models using Ensemble Kalman Filter. SPE Journal.

[64] S. Srinivasan and S. Bryant. SPE 89444: Integrating dynamic data in reservoir models using a parallel computing approach. SPE/DOE Thirteenth Symposium on Improved Oil Recovery, Tulsa, Oklahoma, U.S.A., 17.

[65] A. Tarantola. Inverse Problems Theory: Methods for Data Fitting and Model Parameter Estimation. Elsevier,, New York, 1st edition, 1987.

[66] A. N. Tikhonov. Regularization of ill-posed problems. Dok. Akad. Nauk SSSR, 153:4952;Soiet Math. Dokl., 4(1963), pp. 1624-1627, 1963.

[67] J.R. Valstar, McLaughlin D.B., te Stroet C. B. M., and F.C. van Geer. A representerbased inverse method for groundwater flow and transport applications. Water Resources Research, 40:W05116, doi:10.1029/2003WR002922, 2004.

[68] X.H. Wen and W. H. Chen. SPE 92991. Real-time reservoir model updating using Ensemble Kalman Filter. SPE Reservoir Simulation Symposium, 31 January-2 February 2005, Houston, Texas, U.S.A.

[69] L. Xu and Daley R. Towards a true 4-dimensional data assimilation algorithm: application of a cycling representer algorithm to a simple transport problem. Tellus, 52A:109-128, 2000.

[70] Q. Xu. Representation of inverse covariance by differential operators. Advances in Atmospheric Sciences, 22(2):181-198, 2005.

[71] M. Zafari and A. C. Reynolds. SPE 95750: Assessing the uncertainty in reservoir description and performance predictions with the Ensemble Kalman Filter. SPE Annual Technical Conference and Exhibition, 9 - 12 October, 2005, Dallas, Texas, USA., www.spe.org.

[72] C. Zhangxin. Degenerate two-phase incompressible flow i. existence uniqueness and regularity of a weak solution. J. of Differential Equations, 171:203-232, 2001. 


\section{Vita}

Marco Antonio Iglesias Hernández, son of Marco Antonio Iglesias Flores and Julieta Hernández Cabrera, was born on November 10th in the year 1979, in Mexico City. He received the degree of Physicist at the Universidad Nacional Autónoma de México in February of 2004. He began his graduate work at the University of Texas at Austin in September 2004 and earned the degree of Master of Science in Computational and Applied Mathematics in December of 2006 .

Permanent Address: 4553 Guadalupe St. B207

Austin, TX 78751

This dissertation was typeset with $\mathrm{LT}_{\mathrm{EX}} 2 \varepsilon^{1}$ by the author.

\footnotetext{
${ }^{1} \mathrm{LT}_{\mathrm{E} X} 2 \varepsilon$ is an extension of $\mathrm{LT}_{\mathrm{E}} \mathrm{X}$. $\mathrm{LT}_{\mathrm{E} X}$ is a collection of macros for $\mathrm{T}_{\mathrm{E}} \mathrm{X}$. $\mathrm{T}_{\mathrm{E}} \mathrm{X}$ is a trademark of the American Mathematical Society. The macros used in formatting this dissertation were written by Dinesh Das, Department of Computer Sciences, The University of Texas at Austin, and extended by Bert Kay, James A. Bednar, and Ayman El-Khashab.
} 